TRANSACTIONS OF THE

AMERICAN MATHEMATICAL SOCIETY

Volume 356, Number 1, Pages 73-117

S 0002-9947(03)03206-9

Article electronically published on August 25, 2003

\title{
COMPACT COVERING MAPPINGS BETWEEN BOREL SETS AND THE SIZE OF CONSTRUCTIBLE REALS
}

\author{
GABRIEL DEBS AND JEAN SAINT RAYMOND
}

\begin{abstract}
We prove that the topological statement: "Any compact covering mapping between two Borel sets is inductively perfect" is equivalent to the set-theoretical statement: " $\forall \alpha \in \omega^{\omega}, \aleph_{1}^{L(\alpha)}<\aleph_{1}$ ".
\end{abstract}

The starting point of this work is the following topological problem:

Problem. Is any compact covering mapping between two Borel spaces inductively perfect?

We recall that if $f: X \rightarrow Y$ is a continuous and onto mapping, then:

- $f$ is said to be perfect if the inverse image by $f$ of any compact subset of $Y$ is compact.

- $f$ is said to be compact covering if any compact subset of $Y$ is the direct image of some compact subset of $X$.

- $f$ is said to be inductively perfect if there exists a subset $X^{\prime}$ of $X$ such that the restriction of $f$ to $X^{\prime}$ is a perfect mapping from $X^{\prime}$ onto $Y$.

All spaces considered here will implicitly be supposed to be embedded in $2^{\omega}$. We follow standard logical notation: $\boldsymbol{\Sigma}_{1}^{1}, \boldsymbol{\Pi}_{1}^{1}, \boldsymbol{\Delta}_{1}^{1}$, for the classes of analytic, coanalytic, Borel sets; $\boldsymbol{\Sigma}_{\xi}^{0}, \boldsymbol{\Pi}_{\xi}^{0}$ for the additive and multiplicative Borel classes; $\Sigma_{1}^{1}, \Pi_{1}^{1}, \Delta_{1}^{1}$, $\Sigma_{\xi}^{0}, \Pi_{\xi}^{0}$ for the effective versions of these classes (see [7] for more details). Notice that $\boldsymbol{\Pi}_{2}^{0}=\mathbf{G}_{\delta}$ and $\boldsymbol{\Sigma}_{2}^{0}=\mathbf{K}_{\sigma}$ (i.e. $\sigma$-compact, since our spaces are embedded in $\left.2^{\omega}\right)$.

It is clear that any inductively perfect mapping is compact covering. We present below the main known results for the converse implication:

(A) In ZFC:

- Any compact covering mapping from a $\boldsymbol{\Pi}_{2}^{0}$ space onto any space is inductively perfect.

- Any compact covering mapping from any space onto a $\boldsymbol{\Sigma}_{2}^{0}$ space is inductively perfect.

(B) If we assume $\operatorname{Det}\left(\boldsymbol{\Sigma}_{1}^{1}\right)\left(\boldsymbol{\Sigma}_{1}^{1}\right.$ determinacy), then:

Any compact covering mapping from a $\boldsymbol{\Pi}_{1}^{1}$ space onto a $\boldsymbol{\Pi}_{1}^{1}$ space is inductively perfect.

Received by the editors May 31, 2001.

2000 Mathematics Subject Classification. Primary 03E15; Secondary 03E45, 54H05.

(C)2003 American Mathematical Society 
(C) For all $\alpha \in \omega^{\omega}$, if we assume that $\aleph_{1}^{L(\alpha)}=\aleph_{1}$, then:

There exists a compact covering mapping $f \in \Delta_{1}^{1}(\alpha)$ between two $\Delta_{3}^{0}(\alpha)$ spaces which is not inductively perfect; more precisely, we can choose $f$ : $X \rightarrow Y$ with $X$ and $Y$ Boolean combinations of $\Pi_{2}^{0}(\alpha)$ sets.

(D) For all $\alpha \in \omega^{\omega}$, if we assume that $\aleph_{1}^{L(\alpha)}<\aleph_{1}$, then:

Any compact covering $f \in \Delta_{1}^{1}(\alpha)$ from a $\Pi_{1}^{1}(\alpha)$ space onto a $\Pi_{3}^{0}(\alpha)$ or $\Sigma_{3}^{0}(\alpha)$ space is inductively perfect.

(E) For all $\alpha \in \omega^{\omega}$, if we assume that $\aleph_{2}^{L(\alpha)}<\aleph_{1}$, then:

Any compact covering mapping $f \in \Delta_{1}^{1}(\alpha)$ from a $\Pi_{1}^{1}(\alpha)$ space onto a $\Sigma_{4}^{0}(\alpha)$ space is inductively perfect.

The first result in (A) was proved several years ago by the second author 9] (see also [1]), whereas the second result is a much more recent result due to Ostrovsky 8] (also to Just and Wicke [6], in the non-trivial case where the range space is countable). (B), (C), (D) are proved (or follow from arguments) in [2, 3], 44; and (E) is announced in [5]. In this paper we shall complete this list by the following result (notice the switch in the quantifier's position):

(F) If we assume that for all $\alpha \in \omega^{\omega}, \aleph_{1}^{L(\alpha)}<\aleph_{1}$, then:

Any compact covering mapping from a $\boldsymbol{\Pi}_{1}^{1}$ space onto a $\boldsymbol{\Delta}_{1}^{1}$ space is inductively perfect.

In particular, combining $(\mathrm{C})$ and $(\mathrm{F})$, we obtain the equivalence announced in the abstract, which completely solves the initial problem. However, this does not provide an equivalence for a fixed $\alpha$ as obtained for mappings valued in $\Pi_{3}^{0}(\alpha)$ or $\Sigma_{3}^{0}(\alpha)$ sets, by combining $(\mathrm{C})$ and $(\mathrm{D})$. In fact, a careful analysis of the arguments gives the following more precise result:

$\left(\mathrm{F}^{\prime}\right)$ For all $\alpha \in \omega^{\omega}$ and all $\xi<\omega_{1}^{C K}$, if we assume that $\aleph_{\omega^{\xi}+1}^{L(\alpha)}<\aleph_{1}$, then:

Any compact covering mapping $f \in \Delta_{1}^{1}(\alpha)$ from a $\Pi_{1}^{1}(\alpha)$ space onto a $\Sigma_{\xi}^{0}(\alpha)$ space is inductively perfect.

Notice that by $(\mathrm{E})$ and $(\mathrm{F})$, for $\xi \leq 4\left(\mathrm{~F}^{\prime}\right)$ is not the best possible result.

We shall in fact prove a stronger result, which we now describe, and which will not involve the notions of compact covering and inductively perfect explicitly. For any space $Z$ let $\mathcal{K}(Z)$ denote the space of all nonempty compact subsets of $Z$, endowed with the Hausdorff topology. Notice first that, for the study of our problem, one can (see Theorem 3.1 in 2]) easily reduce the general case to the particular case where $X \subset Y \times 2^{\omega} \subset 2^{\omega} \times 2^{\omega}$ and the mapping $f$ is the restriction to $X$ of the canonical projection $\pi$ on the first factor. Given any such projection mapping $f$, if we set $Z=\mathcal{K}(X) \subset \mathcal{K}\left(2^{\omega}\right)$ and $F=\left\{(y, H) \in 2^{\omega} \times \mathcal{K}\left(2^{\omega}\right): y \in \pi(H)\right\}$, then

$f$ is compact covering

$$
\Longleftrightarrow \forall K \text { compact } \subset Y, \quad \exists z \in Z: K \times\{z\} \subset F \cap(Y \times Z)
$$

and

$f$ is inductively perfect

$$
\Longleftrightarrow \exists g: Y \rightarrow \mathcal{K}\left(2^{\omega}\right) \text { continuous such that } g \subset F \cap(Y \times Z) \text {. }
$$


The first equivalence is just a straightforward reformulation. The second equivalence is not difficult to prove, but we omit the proof because we shall use only its trivial part $(\Longleftarrow)$. Notice that $\mathcal{K}\left(2^{\omega}\right)$ is homeomorphic to $2^{\omega}, F$ is a closed subset of $2^{\omega} \times \mathcal{K}\left(2^{\omega}\right)$, and $Z$ is $\Pi_{1}^{1}$ if $X$ is $\Pi_{1}^{1}$. It is clear then from these observations that statement $(\mathrm{F})$ is a consequence of the following more general result that we will prove:

Main Theorem. Assume that " $\forall \alpha \in \omega^{\omega}, \aleph_{1}^{L(\alpha)}<\aleph_{1}$ ". Let $X=B \cap(Y \times Z)$ be a subset of $2^{\omega} \times 2^{\omega}$ with $Y \subset 2^{\omega}$ Borel, $Z \subset 2^{\omega}$ coanalytic, and $B \subset 2^{\omega} \times 2^{\omega}$ Borel.

If for any compact subset $K$ of $Y$ there exists $z \in Z$ such that $K \times\{z\} \subset X$, then there exists a continuous mapping $f: 2^{\omega} \rightarrow 2^{\omega}$ such that $f_{\left.\right|_{Y}} \subset X$, where $f_{\left.\right|_{Y}}$ denotes the graph of the restriction of $f$ to $Y$.

The proof of this result, which is very long, constitutes practically the whole content of the paper; and we shall try, by the following remarks, to describe its general scheme and some of the basic ideas.

1) As a first approach we shall inspect briefly the proof of the Main Theorem under the stronger assumption $\operatorname{Det}\left(\boldsymbol{\Sigma}_{1}^{1}\right)$, as developed in [2]. In this case one can consider the game $G_{0}$ where two players construct, in a standard Lipschitz way, two elements in $2^{\omega}$ : $y$ constructed by Player $I$, and $z$ constructed by Player II, with the following win condition for Player II: $(y \notin Y$ or $(z \in Z$ and $(y, z) \in B))$ This condition is clearly the difference of two $\boldsymbol{\Pi}_{1}^{1}$ sets, and by a well known result of Martin such games are determined under $\operatorname{Det}\left(\boldsymbol{\Sigma}_{1}^{1}\right)$.

Assume that Player $I$ has a winning strategy $\sigma$ in this game; then the set $K_{0}$ of all points $y$ constructed by Player $I$ in all possible runs in the game is compact (as the image of $2^{\omega}$ by a continuous function). Moreover, it follows easily from the win condition that $K \subset Y$ and that there is no $z$ in $Z$ such that $K \times\{z\} \subset X$ (otherwise Player II can beat $\sigma$ by playing $z$ ). This contradicts the assumptions of the theorem. Hence Player II has a winning strategy which clearly defines a continuous mapping $f: y \mapsto z$; and again the win condition ensures that the graph of the restriction of $f$ to $Y$ is necessarily contained in $B \cap(Y \times Z)$, which proves the theorem.

2) Notice that if in the statement of the theorem we replace along the product space $2^{\omega} \times 2^{\omega}$ by $2^{\omega} \times \omega^{\omega}$, and if we consider a similar game in which Player $I$ constructs some $y \in 2^{\omega}$ and Player II constructs some $z \in \omega^{\omega}$, with the same win condition, then the arguments above do not work any more because the set $K_{0}$ defined above is no longer compact (since at each move Player II has infinitely many possible choices). However, there is a simple way to fix the proof in this context: Consider instead of a classical Ulam type game, the game $G_{1}$ in which Player I constructs in a standard Lipschitz way an element $y \in 2^{\omega}$, whereas Player II constructs an element $z \in \omega^{\omega}$ by playing its successive coordinates from time to time, otherwise the player passes. To win some infinite run in $G_{1}$, Player II has to play (not pass) infinitely many times in the run, defining thus some $z \in \omega^{\omega}$, and then to ensure the same win condition as in $G_{0}$.

Let's say that a position in the game $G_{1}$ is good if for all $n$ the $n^{\text {th }}$ coordinate of $z$ is played by Player II at the $k^{\text {th }}$ move only if $z(n) \leq k$. The set $K_{1}$ of all points $y$ constructed by Player $I$ in all good runs is again compact, since at each step Player II has finitely many possible choices (including the possibility of passing), and we can repeat the previous arguments. 
3) We now drop the strong assumption $\operatorname{Det}\left(\boldsymbol{\Sigma}_{1}^{1}\right)$, and go back to the real statement of the Main Theorem. We shall try to change the win condition of $G_{0}$ slightly to obtain a Borel game. The problem comes of course from the condition " $z \in Z$ ", which is $\Pi_{1}^{1}$, and we have to ensure this condition in some indirect Borel (most reasonably closed) way. The classical solution is then to fix a tree $T$ on $2 \times \omega_{1}$ such that $Z$ is the projection on the first factor of $\lceil T\rceil \subset 2^{\omega} \times \omega_{1}^{\omega}$, and consider the game $G_{2}$ in which we ask Player II to construct, in addition to $z$, a witness $\bar{\rho} \in \omega_{1}^{\omega}$ such that $(z, \bar{\rho}) \in\lceil T\rceil$, which will ensure automatically that $z \in Z$.

Unfortunately, as we shall see, this procedure cannot be applied here. Notice first that it follows from our previous discussion that for our application (to compact covering mappings) one can assume that $B=F$ is a closed set, and that $Y$ is exactly the projection of $X$. Now a winning strategy for Player II in $G_{2}$ defines, as above, a continuous mapping $f: y \mapsto z$ from $2^{\omega}$ to $2^{\omega}$, but also a continuous mapping $g: y \mapsto(z, \bar{\rho})$ from $Y$ to the complete metric (non-separable) space $2^{\omega} \times \omega_{1}^{\omega}$ valued in the the closed subspace $\lceil T\rceil$ and whose graph is contained in $F$. Then one can extend $g$ to a continuous mapping $\tilde{g}$ defined on a $\Pi_{2}^{0}$ space $\tilde{Y} \supset Y$, and necessarily $\tilde{g}$ is also valued in $\lceil T\rceil$ and its graph is contained in $F$; it follows that $\tilde{Y}$ is contained in the projection $X$, hence that $\tilde{Y}=Y$, and in particular that $Y$ is $\mathbf{\Pi}_{2}^{0}$, which we did not suppose a priori. This also explains why the original problem is easier to study when the range space is supposed to be $\boldsymbol{\Pi}_{2}^{0}$.

The same argument shows that to prove the Main Theorem by such a variation of the game $G_{0}$, the construction of the witness by Player II should yield to a function which is at least of the (multiplicative) Borel class of $Y$.

4) For all $z \in 2^{\omega}$, set $T(z)=\left\{\rho \in \omega_{1}^{<\omega}: \exists k<\omega,\left(\rho, z_{\left.\right|_{k}}\right) \in T\right\}$; we shall now define a game $G$ in which we ask Player II , in addition to the construction of $z$ as above, to choose from time to time some $\rho \in \omega_{1}^{<\omega} \cap T(z)$, producing thus a finite or infinite sequence $\theta=\left(\rho_{n}\right)_{n \in N}$ in $\omega_{1}^{<\omega}$. We shall not ask that $\rho_{n} \prec \rho_{n+1}$, but rather impose that Range $(\theta)=\left\{\rho_{n} ; n \in N\right\}$ is a subtree of $T(z)$, and then we shall arrange things so that whenever $y \in Y$ and $N$ is infinite, then Range $(\theta)$ has an infinite branch; it will follow a fortiori that $T(z)$ has an infinite branch, and this will ensure again that $z \in Z$, as desired.

To realize this program we use a tree representation for the set $Y$, that is, a continuous function which associates to any $y \in 2^{\omega}$ a tree $R^{+}(y)=\left\{r_{n} ; n \in \omega\right\}$, given with an enumeration, in such a way that

$$
y \in Y \Longleftrightarrow R^{+}(y) \text { is ill-founded } \Longleftrightarrow R^{+}(y) \text { has a unique branch. }
$$

More precisely, if such a representation is fixed, then one can consider the game $G$ in which the players construct $y, z$, and $\theta$ as above, so that (with the previous notation) the mapping $\varepsilon: r_{n} \mapsto \rho_{n}$ defines a tree embedding of the tree $\left\{r_{n} ; n \in N\right\} \subset R^{+}(y)$ in the tree $T(z)$. Player $I I$ wins the run in $G$ if $N$ is infinite (so that the domain of $\varepsilon$ is the whole tree $\left.R^{+}(y)\right)$ and if moreover, as in $G_{0},(y \notin Y$ or $(y, z) \in B)$; thus, if $y \in Y$, then the tree $R^{+}(y)$ will have an infinite branch, hence $T(z)$ also; and this will ensure that $z \in Z$.

5) Fix some strategy $\sigma$ for Player $I$ in $G$. For simplicity we shall supose that all the parameters of the game are in $L$. The first step is to reduce the problem to the case where the strategy $\sigma$ is also in $L$; this is obtained by a general absoluteness argument, although $G$ is not a game on the integers. Then we shall define, from $\sigma$, a compact set $K$ with similar properties to $K_{0}$. Notice that, because of the ordinal 
part in the game, the set of all points $y$ played by Player $I$ in $G$ is not a priori compact.

In fact, we shall define a large family of special finite positions in the game, compatible with $\sigma$, that we shall call admissible positions, and then define $K$ as the set of all points $y \in 2^{\omega}$ obtained as limits of finite sequences $s \in 2^{<\omega}$ constructed by Player $I$ in some arbitrary admissible position; so that by its very definition $K$ is compact. Notice that we do not require that the elements of $K$ be constructed by Player $I$ in some infinite run in the game; but we shall arrange that $K$ contains enough such points. More precisely we shall ensure that:

a) Any $y \in K \backslash Y$ can be played by Player I in an infinite run compatible with $\sigma$.

In particular, if $\sigma$ is a winning strategy, then it follows from the win condition of the game that $K$ is a compact subset of $Y$. We shall also ensure that:

b) For any $(z, \bar{\rho}) \in\lceil T\rceil$ there exists an infinite run compatible with $\sigma$, in which Player I constructs an element $y \in K$, and Player II constructs $z$ and a tree embedding $\varepsilon: R^{+}(y) \rightarrow T(z)$ which sends the unique branch of $R^{+}(y)$ onto $\bar{\rho}$.

Hence, if $\sigma$ is a winning strategy, then there exists no $z \in Z$ such that $K \times\{z\} \subset X$. So $K$ is large enough to contain the answers by $\sigma$ to these particular runs (compare this with the definition of the compact set $K_{0}$ in Remark 1).

This proves that under the hypothesis of the Main Theorem Player I has no winning strategy in the game $G$. Hence by Borel determinacy Player II has a winning strategy in this game; and one can repeat the arguments for $G_{0}$ to derive the conclusion of the theorem.

6) It is clear that to ensure a) above one needs to have some control on the set $Y^{c}$ (the complement of $Y$ in $2^{\omega}$ ); so in addition to the tree representation $R^{+}$for $Y$, we also fix a tree representation $R^{\bullet}$ for $Y^{c}$. To understand this notation one should think of $Y$ as being in some additive Borel class and hence $Y^{c}$ in the corresponding multiplicative Borel class. Moreover, $R^{+}$and $R^{\bullet}$ have to be compatible in some technical sense; such a pair of trees $\left(R^{+}, R^{\bullet}\right)$ will be called a double tree.

But to ensure $b$ ) we need to work with more special double trees, which we will call interpolable, and which provide a transfinite family $\left(R^{\xi}\right)_{\xi<\omega_{1}}$ of trees, describing in some vague sense the history of the construction of the double tree $\left(R^{+}, R^{\bullet}\right)$.

The existence of such a double tree representation for any Borel set is a totally non-trivial fact that constitutes a large part of the paper, and is of some intrinsic interest.

Finally we mention that the notion of tree used here is to be understood in the abstract sense, as a partial order such that the set of all predecessors of any element is finite and totally ordered. For example, each of $R^{+}, R^{\bullet}, R^{\xi}$ above will in fact be a binary relation on the set $2^{<\omega}$. But these details are irrelevant in this general discussion.

7) We finish with some comments about the proofs of properties a) and b) above. Notice that in both cases one has to construct some infinite run in the game.

For a) this is obtained by a compactness argument: The first naive idea is to require that admissible positions be "good" in the sense of Remark 2, but the problem is that, unlike in the game $G_{1}$, in the game $G$ Player II 's moves are in an uncountable space. This is treated by introducing on the set $W$ of all positions an equivalence relation $\tilde{E}$ which, because of the assumption " $\forall \alpha \in \omega^{\omega}, \aleph_{1}^{L(\alpha)}<\aleph_{1}$ ", 
will have countably many classes; and this will be sufficient to formulate a notion of "goodness", in particular to ensure that the set of all good positions of a given length is finite. Notice that because of $b$ ) we cannot realize our initial goal strictly, and an admissible position $u$ will not necessarily be good; but, from the definition of admissibilty, we shall be able to associate to $u$ a sequence $\left(u^{(j)}\right)_{j \leq n}$ of good positions satisfying some compatibilty conditions.

For b) the construction of the infinite run will follow totally different ideas, and can be viewed as a converging limit procedure. In particular, unlike the previous case where the run is finally obtained by abstract extractions, putting together all the successives steps of the proof of $b$ ), one could obtain a (very long) explicit definition for the run, which is Borel in the parameters. Details are very technical, and it is very difficult to give the main ideas at this level of generality. The reader might get the flavor by consulting the particular cases (where $Y$ is $\boldsymbol{\Pi}_{3}^{0}$ or $\boldsymbol{\Sigma}_{3}^{0}$ ) treated in [4] and [5]. We mention only that one needs to work with a new family $V \subset W$ of positions. Good positions will be in $V$ by definition, but this will not the case for admissible positions; however, in the proof of $b$ ) we will need to construct admissible positions which in addition belong to $V$.

Notice that neither a) nor $b$ ) is proved by induction. But the definition of the equivalence relation $\tilde{E}$ and the family $V$ will be the result of an inductive construction. More precisely, we shall construct inductively for all $\xi<\omega_{1}$ an equivalence relation $\tilde{E}_{\xi}$ on $W$, and a subset $V_{\xi}$ of $W$ on which $\tilde{E}_{\xi}$ behaves nicely, and then define $V=\bigcap_{\xi} V_{\xi}$ and $\tilde{E}=\bigcap_{\xi} \tilde{E}_{\xi}$. This construction is intimately related to the interpolation family $\left(R^{\xi}\right)_{\xi<\omega_{1}}$ given by the double tree representation. One can even say that the interpolation property is precisely what is needed for this construction to work.

As a final observation, we point out that the way the assumption " $\forall \alpha \in \omega^{\omega}$, $\aleph_{1}^{L(\alpha)}<\aleph_{1}$ " is used in this proof does not match with other classical uses of this assumption. A typical example is the proof of the perfect set theorem for $\boldsymbol{\Pi}_{1}^{1}$ sets: Assuming that some $\Pi_{1}^{1}$ set $X$ is thin (contains no perfect set), one can define in an absolute way (in $L$ ) a set $A \supset X$ such that, if ever $\aleph_{1}^{L}<\aleph_{1}$, then $A$ is countable, and hence $X$ also. The same scheme applies for the proof of the Baire property for $\Pi_{1}^{1}$ sets. In this proof the set-theoretical assumption is used to construct the compact set $K$ and not to prove a) or b), and in particular the set $K$ is not in $L$.

The paper is naturally composed of two distinct parts. In the first part, using the double tree representation theorem, we prove the Main Theorem and give some applications; the second part is devoted to the proof of the double tree representation theorem and is totally independent of the first part.

The first part is organized as follows. In Section 1 we give some applications of the Main Theorem, namely to properties of $\boldsymbol{\Pi}_{1}^{1}$ cofinal sets in hyperspaces of compact sets. All general notation and preliminaries are introduced in Section 2 in particular, the reader will find in this section a brief presentation of the notion of interpolable double tree and the statement (without proof) of the double tree representation theorem for an arbitrary Borel set. In Section 3 we introduce the game $G$ and reduce the proof of the Main Theorem to a general result (Main Lemma 3.1) on this game, which essentially states properties a) and b) considered above. Strictly speaking, the Main Lemma will be proved only in Section 6. One of the main ingredients of this proof is an analysis of the strategies of Player I which reflects the double tree structure, and more particularly the interpolation 
property. This analysis is introduced in Section 4 which is essentially notational and does not contain any new result, but is fundamental for the sequel. The other ingredient of the proof is an inductive construction that we shall develop separately in Section [5, namely the construction of the families $\left(V_{\xi}\right)$ and $\left(\tilde{E}_{\xi}\right)$ considered above. The definitions and properties of these families are very technical; also, for better readability we collected formally all the results of this section that are needed for the proof of the Main Lemma in the next section, in one result (Theorem 5.1) which is stated at the beginning of Section 5 .

The second part is constituted by the last three sections. In Section 7 we come back to the notion of double tree and establish a number of technical properties that we shall use. In Section 8 we define the product operation $\otimes$ on sequences of double trees, which is the basic operation by which we associate inductively a double tree to any Borel set. In Section 9 we introduce the notion of interpolability for a double tree, and prove the existence of a canonical interpolation family obtained by an explicit derivation operation; finally we prove the main result asserting that interpolability is preserved by the product operation $\otimes$, from which the representation theorem follows easily.

\section{Applications}

In this section we go back to the original topological problem discussed in the introduction, and give several other equivalent but more descriptive formulations. For this we need to fix some notation.

For any space $Z$ we denote by $\mathcal{K}(Z)$ the hyperspace of all compact subsets of $Z$, endowed with the Hausdorff topology (inherited from the Hausdorff topology of $\left.\mathcal{K}\left(2^{\omega}\right)\right)$.

Let $A$ and $B$ be two subsets of $\mathcal{K}(Z)$. A mapping $\Phi: A \rightarrow B$ is said to be a domination function if for all $S \in A, \Phi(S) \supset S$. If such a mapping $\Phi$ exists, we shall say that $B$ admits a domination function on $A$. A subset $C$ of a $\mathcal{K}(Z)$ is said to be cofinal if it is a cofinal subset of $\mathcal{K}(Z)$ for the inclusion relation $\subset$ (i.e. $\forall S \in \mathcal{K}(Z), \exists T \in C$ such that $S \subset T$ ). We shall say that $C$ is Borel (continuously) cofinal if $C$ admits a Borel (continuous) domination function on $\mathcal{K}(Z)$. In [3] we proved that:

If $Z$ is $\boldsymbol{\Pi}_{2}^{0}$, then any analytic cofinal subset of $\mathcal{K}(Z)$ is continuously cofinal.

Notice that if a hyperspace $\mathcal{K}(Z)$ contains an analytic cofinal subset $C$, then we can write $\mathcal{K}(Z)=\left\{S \in \mathcal{K}\left(2^{\omega}\right): \exists T \in C\right.$ such that $\left.S \subset T\right\}$; hence $\mathcal{K}(Z)$ is also analytic, and (see [9] or [1]) $Z$ is necessarily $\boldsymbol{\Pi}_{2}^{0}$. So when $Z$ is a Borel (not $\boldsymbol{\Pi}_{2}^{0}$ ) or coanalytic space, the simplest cofinal subsets are $\boldsymbol{\Pi}_{1}^{1}$.

We now define the other notion we shall use: If $X$ is a subset of some product space $Y \times Z$, and $M$ is a subset of $Y$, a lifting of $M$ in $X$ is a mapping $g: M \rightarrow Z$ whose graph is contained in $X$.

Theorem 1.1 and Theorem 1.2, below, which we state for completeness, are from [4] and [3. We denote by $\mathbb{Q}$ the set of all rational numbers, and by $\leq^{*}$ the partial order of eventual domination in $\omega^{\omega}:\left(\alpha \leq^{*} \beta \Longleftrightarrow \exists n \forall m \geq n, \alpha(m) \leq \beta(m)\right)$.

Theorem 1.1. The following are equivalent:

(i) $\operatorname{Det}\left(\boldsymbol{\Sigma}_{1}^{1}\right)$

(ii) For any $\Pi_{1}^{1}$ set $X \subset Y \times 2^{\omega}$ with $Y \boldsymbol{\Pi}_{1}^{1}$, if any compact subset of $Y$ admits a continuous lifting in $X$, then $Y$ admits a continuous lifting in $X$. 
(iii) Any $\boldsymbol{\Pi}_{1}^{1}$ cofinal subset of $\mathcal{K}(Z)$ with $Z \mathbf{\Pi}_{1}^{1}$ is continuously cofinal.

(iv) Any $\boldsymbol{\Pi}_{1}^{1}$ cofinal subset of $\mathcal{K}(\mathbb{Q})$ is Borel cofinal.

(v) Any compact covering mapping from a $\boldsymbol{\Pi}_{1}^{1}$ space onto a $\boldsymbol{\Pi}_{1}^{1}$ space is inductively perfect.

Theorem 1.2. The following are equivalent:

(i) For all $\alpha \in \omega^{\omega}$ the set $\omega^{\omega} \cap L(\alpha)$ is $\leq^{*}$-bounded in $\omega^{\omega}$.

(ii) For any $\boldsymbol{\Pi}_{1}^{1}$ subset $X$ of $Y \times 2^{\omega}$ with $Y \boldsymbol{\Pi}_{2}^{0}$, if any compact subset of $Y$ admits a continuous lifting in $X$, then $Y$ admits a continuous lifting in $X$.

(iii) Any $\boldsymbol{\Pi}_{1}^{1}$ cofinal subset of $\mathcal{K}(Z)$ with $Z \boldsymbol{\Pi}_{2}^{0}$, is continuously cofinal.

(iv) Any $\boldsymbol{\Pi}_{1}^{1}$ cofinal subset of $\mathcal{K}\left(\omega^{\omega}\right)$ is Borel cofinal.

(v) Any compact covering mapping from a $\boldsymbol{\Pi}_{1}^{1}$ space onto a $\boldsymbol{\Pi}_{2}^{0}$ space is inductively perfect.

Theorem 1.3. The following are equivalent:

(i) For all $\alpha \in \omega^{\omega}$ the set $\omega^{\omega} \cap L(\alpha)$ is countable.

(ii) For any $\boldsymbol{\Pi}_{1}^{1}$ subset $X$ of $Y \times 2^{\omega}$ with $Y \boldsymbol{\Delta}_{1}^{1}$, if any compact subset of $Y$ admits a continuous lifting in $X$, then $Y$ admits a continuous lifting in $X$.

(iii) Any $\boldsymbol{\Pi}_{1}^{1}$ cofinal subset of $\mathcal{K}(Z)$ with $Z \boldsymbol{\Pi}_{1}^{1}$ admits a continuous domination function on any $\boldsymbol{\Sigma}_{1}^{1}$ subset of $\mathcal{K}(Z)$.

(iv) Any $\boldsymbol{\Pi}_{1}^{1}$ cofinal subset of $\mathcal{K}(Z)$ with $Z \boldsymbol{\Delta}_{1}^{1}$ admits a continuous domination function on the set of all singletons in $Y$.

(v) Any compact covering mapping from a $\boldsymbol{\Pi}_{1}^{1}$ space onto a $\boldsymbol{\Delta}_{1}^{1}$ space is inductively perfect.

(vi) Any compact covering mapping defined on a $\boldsymbol{\Delta}_{1}^{1}$ space is inductively perfect.

Proof. $(i) \Longrightarrow($ ii $)$ : Follows from Theorem 3.2 by an argument which is similar to the proof of Theorem 7.4 in 4 .

$($ ii $) \Longrightarrow($ iii $)$ : Let $C$ be a $\boldsymbol{\Pi}_{1}^{1}$ cofinal subset in $\mathcal{K}(Z)$ with $Z \boldsymbol{\Pi}_{1}^{1}$. By the separation theorem any $\boldsymbol{\Sigma}_{1}^{1}$ subset of $\mathcal{K}(Y)$ is contained in a Borel subset of $\mathcal{K}(Z)$. So it is enough to prove that $C$ admits a continuous domination function on any Borel subset $B$ of $\mathcal{K}(Z)$. For this, set $A=\{(S, T) \in B \times C: S \subset T\}$ and notice that if $K$ is a compact subset of $B$, then $S_{0}=\bigcup K$ is a compact subset of $Z$, and since $C$ is cofinal, $S_{0}$ is contained in some compact set $T_{0} \in C$; hence $K \times\left\{T_{0}\right\} \subset A$, and the constant function defined on $K$ by $S \mapsto T_{0}$ is a continuous lifting of $K$ in $A$. Then applying $(i)$ we get a continuous lifting of $B$ in $A$, which clearly defines a continuous domination function on $B$ for $A$.

$($ iii $) \Longrightarrow($ iv $)$ : Obvious.

$(i v) \Longrightarrow(v)$ : Straightforward; see the proof of $(i i i) \Longrightarrow(i)$ in Theorem 6.4 in 3 .

$(v) \Longrightarrow(i)$ : Follows from Theorem 7.2 in 4 .

$(i) \Longrightarrow(v i)$ : By $([2]$, Theorem 8.8), (i) implies that the range space of any compact covering mapping defined on a Borel space is also Borel; and it follows from the implication $(i) \Longrightarrow(v)$ (already proved) that such a mapping is inductively perfect.

$(v i) \Longrightarrow(v)$ : This follows from the proof of Theorem 6.5 in [3], which is stated for mappings with $\boldsymbol{\Pi}_{2}^{0}$ range, but a simple check shows that the arguments are also valid for mappings with $\boldsymbol{\Delta}_{1}^{1}$ range.

We do not know any direct proof for $(i) \Longrightarrow($ iii $)$. 
The reader can find in the statement of Theorem 7.2 in [4] a complement to the list of equivalences of Theorem 1.3 above.

1.4. Open problems. 1) We reformulate here a problem raised in the introduction: We recall that both Theorem 1.1 and Theorem 1.2 admit effective versions (obtained by replacing all descriptive classes in the statements by their corresponding effective version, and fixing $\alpha=0$ ). But we do not know of similar versions for Theorem 1.3.

2) As mentioned in the proof of Theorem[1.3 under the assumption " $\forall \alpha, \aleph_{1}^{L(\alpha)}<$ $\aleph_{1}$ " one can prove $([2]$, Theorem 8.8$)$ that:

The image of a Borel set by a compact covering mapping is also Borel.

But we do not know whether this statement is provable in $Z F C$. This is true for simple Borel sets: It is proved in (2], Theorem 5.2) that if $f: X \rightarrow Y$ is compact covering and $X$ is the intersection of a $\boldsymbol{\Pi}_{2}^{0}$ set and a $\boldsymbol{\Sigma}_{2}^{0}$ set, then $Y$ is a Borel set of the same type, though in this case $f$ is not necessarily inductively perfect ([3], Theorem 6.5). We mention also that by ([2], Theorem 8.6) if $f: X \rightarrow Y$ is compact covering and $X$ and $Y$ are Borel, then $Y$ is in the same (additive or multiplicative) Borel class as $X$.

\section{Preliminaries}

2.1. Sequential spaces. For any set $A$ we denote by $\operatorname{Seq}(A)$ the set of all finite sequences in $A$, by $\prec$ the strict extension relation in $\operatorname{Seq}(A)$, and by $\preceq$ or Ext the large extension relation, that is,

$$
s \preceq t \Longleftrightarrow s \prec t \text { or } s=t .
$$

As usual, if $s \in \operatorname{Seq}(A)$ we denote by $|s|$ the length of the sequence $s$, and for any $k \leq|s|$ by $s_{\left.\right|_{k}}$ the restriction of $s$ to $k$; and for $k<|s|$ we shall also use very often the notation $s_{\uparrow_{k}}$ for the restriction of $s$ to $k+1$, thus

$$
s_{\uparrow_{k}}=s_{\left.\right|_{k+1}} \text {. }
$$

We shall also consider in this work the space of partial functions from $\omega$ to $A$ with finite domain. We shall denote by $\subset$ the extension relation in this space. If $\tau$ is such a function, we shall denote by $\operatorname{Dom}(\tau)$ its domain, which is a finite subset of $\omega$, and by $\tau^{*}$ the function obtained from $\tau$ by deleting from $\operatorname{Dom}(\tau)$ its largest element. If $\jmath: \operatorname{card}(\operatorname{Dom}(\tau)) \rightarrow \operatorname{Dom}(\tau)$ is the unique increasing enumeration of $\operatorname{Dom}(\tau)$, then the sequence $\theta=\tau \circ \jmath \in \operatorname{Seq}(A)$ will be called the sequential rearrangement of $\tau$. It is clear that the pair $(\operatorname{Dom}(\tau), \theta)$ entirely determines $\tau$. In particular, if $s \in \operatorname{Seq}(A)$ then $s^{*}=s_{|s|-1}$.

When the set $A$ is endowed with some wellordering $\leq$, then the set $\operatorname{Seq}(A)$ can also be canonically equipped with the corresponding lexicographical wellordering, which we also denote by $\leq$, defined for $s, t \in \operatorname{Seq}(A)$ by:

$$
s \leq t \Longleftrightarrow\left\{\begin{array}{l}
s \preceq t \\
\text { or } \\
\exists k<|s|, \text { such that } s_{\left.\right|_{k}} \prec t \text { and } s(k)<t(k)
\end{array}\right.
$$

where, as usual, $<$ denotes the strict order corresponding to $\leq$. Notice that one can then start again from the wellordered set $\operatorname{Seq}(A)$ to wellorder the set $\operatorname{Seq}(\operatorname{Seq}(A))$, and so on. In this paper we shall apply this procedure to wellorder the sets $\operatorname{Seq}\left(\omega_{1}\right)$ and $\operatorname{Seq}\left(\operatorname{Seq}\left(\omega_{1}\right)\right)$. 
The fundamental property of lexicographical wellordering, which we shall use frequently, is the following:

If $s \preceq s^{\prime}, t \preceq t^{\prime},|s|=|t|$ and $s<t$, then $s^{\prime}<t^{\prime}$.

2.2. Trees. A tree relation $R$ on a set $E$ is an order relation with a least element such that for any $s \in E$ the initial segment $\{t \in E: t R s\}$ is finite and linearly ordered by $R$.

The height of an element $s \in E$, denoted by $h_{R}(s)$, is the number of elements strictly smaller than $s$ :

$$
h_{R}(s)=\operatorname{card}(\{t \in E: t \neq s \text { and } t R s\}) .
$$

If $s R t$ with $h_{R}(t)=h_{R}(s)+1$, we say that $t$ is a successor of $s$, or $s$ is the predecessor of $t$.

If $R^{\prime}$ is also a tree relation on a set $E^{\prime}$, a tree homomrphism from $(E, R)$ to $\left(E^{\prime}, R^{\prime}\right)$ is a mapping $f: E \rightarrow E^{\prime}$ satisfying, for all $s, t \in E$,

$$
s R t \Longrightarrow f(s) R^{\prime} f(t) \quad \text { and } \quad h_{R^{\prime}}(f(s))=h_{R}(s) .
$$

By a standard tree on a set $A$ we shall mean a subset $T \subset \operatorname{Seq}(A)$ which is left hereditary for the extension relation $\preceq$; this is the most classical notion of tree. Notice that for a standard tree $T$ the height of an element $s \in T$ is exactly $|s|$, the length of the sequence $s$.

Most of the tree relations we shall consider in this paper will have as domain a sequential space Seq $(A)$, but will be different from the extension relation $\preceq$.

A tree relation $R$ on the sequential space Seq $(A)$ is said to be an adapted tree if $R$ is finer than the extension relation $\preceq$ :

$$
s R t \Longrightarrow s \preceq t .
$$

For such an $R$ we clearly have $h_{R}(s) \leq|s|$, for any $s \in \operatorname{Seq}(A)$. In particular, $h_{R}(\emptyset)=0$, and the empty sequence $\emptyset$ is always the least element.

Definition 2.3. A double tree on a set $A$ is a pair $\mathcal{R}=\left(R^{+}, R^{\bullet}\right)$ of adapted tree relations on $\operatorname{Seq}(A)$ satisfying:

Any two $R^{+}$-comparable elements in Seq $(A) \backslash\{\emptyset\}$ have the same $R^{\bullet}$-predecessor.

We denote by $h^{+}=h_{\mathcal{R}}^{+}$and $h^{\bullet}=h_{\mathcal{R}}^{\bullet}$ the height functions on Seq $(A)$ with respect to $R^{+}$and $R^{\bullet}$.

A detailed study of double trees will be developed in Section 7 for the moment we restrict ourselves to two simple examples, and some basic general properties that we will use in the proof of the Main Theorem. But before this, let's recall that if $R_{0}$ and $R_{1}$ are two relations on a set $E$, then $R_{0} \circ R_{1}$ denotes the relation $R$ defined by

$$
s R t \Longleftrightarrow \exists u \in E \text { such that } s R_{0} u \text { and } u R_{1} t,
$$

Notice that if $R_{0}$ and $R_{1}$ are reflexive, then $R$ is also reflexive, and both $R_{0}$ and $R_{1}$ are finer than $R$, so $R_{0} \cup R_{1} \subset R_{0} \circ R_{1}$. But in general, transitivity and the tree property are preserved neither by union nor by composition. 
Example 2.4. If $Y$ is a $\Sigma_{1}^{0}$ subset of $A^{\omega}$, and $Y^{c}=\lceil T\rceil$ for some standard tree $T$ on $A$, we can define a a canonical double tree $\mathcal{R}=\left(R^{+}, R^{\bullet}\right)$ in Seq $(A)$ by

$$
\begin{aligned}
s R^{+} t & \Longleftrightarrow(s=\emptyset \quad \text { or } \quad s=t \quad \text { or } \quad(s \prec t \text { and } s \notin T)), \\
s R^{\bullet} t & \Longleftrightarrow(s=t \quad \text { or } \quad(s \prec t \text { and } s \in T)) .
\end{aligned}
$$

One can also easily check that $\left(R^{+}, R^{\bullet}\right)$ is a double tree, and, moreover,

$$
\left\{\begin{array}{l}
R^{\bullet} \cap R^{+}=\Delta_{0}:=\{(s, t): s=\emptyset \text { or } s=t\} \\
R^{\bullet} \cup R^{+}=R^{\bullet} \circ R^{+}=R^{+} \circ R^{\bullet}=\text { Ext }
\end{array}\right.
$$

We recall that Ext denotes the extension $\preceq$ relation on Seq $(A)$.

Example 2.5. If $Y$ is a $\boldsymbol{\Sigma}_{2}^{0}$ subset of $A^{\omega}$, we can fix a sequence $\left(T_{i}\right)$ of standard trees $T_{i}$ on $A$ such that $Y=\bigcup_{i}\left\lceil T_{i}\right\rceil$ and $\operatorname{Seq}(A)=\bigcup_{i} T_{i}$. We shall define a canonical double tree $\mathcal{R}=\left(R^{+}, R^{\bullet}\right)$ in $\operatorname{Seq}(A)$. For any $t \in \operatorname{Seq}(A)$ and any $i \in \omega$ let

Then set

$$
k_{i}=\max \left\{m \leq|t|: t_{\left.\right|_{m}} \in T_{i}\right\} \quad \text { and } \quad t^{(i)}=t_{\left.\right|_{k_{i}}} .
$$

$$
t^{\prime}= \begin{cases}t^{(k)} & \text { if } M:=\left\{k_{i}: k_{i}<|t|, i \in \omega\right\} \neq \emptyset \text { and } k=\max M \\ \emptyset & \text { if } M=\emptyset\end{cases}
$$

and define

$$
\begin{aligned}
& s R^{+} t \Longleftrightarrow\left(s=\emptyset \quad \text { or } \quad t^{\prime} \prec s \preceq t\right), \\
& s R^{\bullet} t \Longleftrightarrow\left(s=\emptyset \quad \text { or } \quad s=t \quad \text { or } \quad\left(s \prec t \text { and } \exists i: s=t^{(i)}\right)\right) .
\end{aligned}
$$

One can also easily check that $\left(R^{+}, R^{\bullet}\right)$ is a double tree, and, moreover,

$$
\left\{\begin{array}{l}
R^{\bullet} \cap R^{+}=\Delta_{0} \\
R^{\bullet} \cup R^{+}=R^{\bullet} \circ R^{+} \subset R^{+} \circ R^{\bullet}=\text { Ext }
\end{array}\right.
$$

In the general situation we have the following basic relations:

Lemma 2.6. Let $\mathcal{R}=\left(R^{+}, R^{\bullet}\right)$ be a double tree on $A$; then:

a) $R^{\bullet} \cap R^{+}=\Delta_{0}$

b) $R^{\bullet} \cup R^{+}=R^{\bullet} \circ R^{+}$,

c) $R:=R^{+} \circ R^{\bullet}$ is an adapted tree relation on $\operatorname{Seq}(A)$, and

d) for any $t \in \operatorname{Seq}(A) \backslash\{\emptyset\}$ :

- If $h^{+}(t)=1$, then the $R$-predecessor of $t$ is its $R^{\bullet}$-predecessor.

- If $h^{+}(t)>1$, then the $R$-predecessor of $t$ is its $R^{+}$-predecessor.

Proof. a) Suppose that for $\emptyset \neq s \neq t$ we have $s R^{+} t$ and $s R^{\bullet} t$. Then, if $s^{\bullet}$ and $t^{\bullet}$ denote the $R^{\bullet}$-predecessors of $s$ and $t$, we have $s R^{\bullet} t^{\bullet}$, and $s^{\bullet}=t^{\bullet}$ by the double tree property; hence $s$ satisfies $s R^{\bullet} s^{\bullet}$, which is impossible.

b) Since $R^{+}$and $R^{\bullet}$ are reflexive, then $R^{\bullet} \cup R^{+} \subset R^{\bullet} \circ R^{+}$. Conversely, suppose that $s R^{\bullet} \circ R^{+} t$, and fix $u$ such that $s R^{\bullet} u$ and $u R^{+} t$ : If $s=u$ then $s R^{+} t$; and if $s \neq u$ then, since $t$ and $u$ are $R^{+}$-comparable, by the double tree property we also have $s R^{\bullet} t$.

We now prove c) and d) simultaneously. Let $\tilde{R}$ denote the preorder relation generated by $R^{+}$and $R^{\bullet}$; we clearly have $R^{+} \cup R^{\bullet} \subset R^{+} \circ R^{\bullet} \subset \tilde{R}$. If $s \tilde{R} t$, then 
there exists a chain $\left(s_{i}\right)_{0 \leq i \leq n}$ of minimal length, such that $s_{0}=s, s_{n}=t$, and, for all $i<n, s_{i} R^{i} s_{i+1}$ with $R^{i}=R^{+}$or $R^{\bullet}$. Then necessarily $n \leq 2$; for otherwise we can find some $i<n-1$ such that $R^{i}=R^{\bullet}$ and $R^{i+1}=R^{+}$, but then by b) we could delete the element $s_{i+1}$ from the chain, which contradicts the minimality of $n$. Hence $\tilde{R} \subset\left(R^{+} \circ R^{\bullet}\right) \cup\left(R^{\bullet} \circ R^{+}\right)=R^{+} \circ R^{\bullet}$, and so $\tilde{R}=R$; and in particular $R$ is a preorder. Moreover, since $R^{+}$and $R^{\bullet}$ are finer than $\preceq$, then the same holds for $R$, and for any $t$, the set $\{s: s R t\}$ is finite.

It follows from Definition 2.3 that $\emptyset$ is the least element for $R$. Fix $t$ in Seq $(A) \backslash\{\emptyset\}$; it is clear that any $R$-predecessor of $t$ is either its $R^{+}$-predecessor or its $R^{\bullet}$-predecessor:

- If If $h^{+}(t)=1$, then the $R^{\bullet}$-predecessor is the unique $R$-predecessor of $t$.

- If If $h^{+}(t)>1$, let $u$ and $v$ denote the $R^{+}$- and $R^{\bullet}$-predecessors of $t$; then by the double tree property we have $v R^{\bullet} u R^{+} t$, and $u$ is the unique $R$-predecessor of $t$.

In particular, any element in Seq $(A) \backslash\{\emptyset\}$ has a unique $R$-predecessor, and $R$ is a tree.

Definition 2.7. A double tree $\mathcal{R}=\left(R^{+}, R^{\bullet}\right)$ on $A$ is said to be interpolable if there exists a family $\left(R^{\xi}\right)_{\xi<\omega_{1}}$ of tree relations on $\operatorname{Seq}(A)$ satisfying:

(i) $R^{0}=$ Ext, $R^{\xi+1} \subset R^{\xi}$, and $R^{\lambda}=\bigcap_{\eta<\lambda} R^{\eta}$ if $\lambda$ is limit.

(ii) For all $s \preceq t \preceq u$ in Seq $(A)$, if $s R^{\xi+1} u$ and $t R^{\xi} u$, then $s R^{\xi+1} t$.

(iii) $\bigcap_{\xi<\omega_{1}} R^{\xi}=R$ is the tree generated by $R^{+}$and $R^{\bullet}$.

Such a family $\left(R^{\xi}\right)_{\xi<\omega_{1}}$ is called an interpolation family for $\mathcal{R}$.

Notice that when $A$ is countable, which will always be the case in the sequel, then each $R^{\xi}$ is also countable; hence it follows from (i) that there exists $\gamma<\omega_{1}$ such that $R^{\gamma}=R$.

The intuition of condition (ii) is not immediate and was initially motivated by the needs of the proof of Theorem 3.2. However, one can observe that this condition ensures that, as a tree, $R^{\xi+1}$ is large inside $R^{\xi}$ : In fact, if in the statement of (ii) one replaces the subformula " $t R^{\xi} u$ " by " $t R^{\xi+1} u$ ", then one obtains a weaker statement (since $R^{\xi+1} \subset R^{\xi}$ ), which expresses exactly that $R^{\xi+1}$ is a tree relation.

In fact, as we shall see in Section 9, from any adapted tree $R$ we shall explicitly define a family $\mathfrak{R}=\left(R^{\xi}\right)_{\xi<\omega_{1}}$ such that if $R$ is interpolable, then $\mathfrak{R}$ is an interpolation family for $R$.

In Section 9 we shall prove a stronger version of the following result:

Theorem 2.8. For any Borel subset $Y$ of $2^{\omega}$ there exists an interpolable double tree $\mathcal{R}=\left(R^{+}, R^{\bullet}\right)$ satisfying:

a) For any $y \in Y$, the set $\{s \in \operatorname{Seq}(2): s \prec y\}$ contains an infinite chain for $R^{+}$.

b) For any $y \notin Y$, the set $\{s \in \operatorname{Seq}(2): s \prec y\}$ contains an infinite chain for $R^{\bullet}$.

Moreover, if $Y$ is $\Delta_{1}^{1}(\alpha)$ for some parameter $\alpha$, then the double tree $\mathcal{R}$ and its interpolation family $\mathfrak{R}$ can be constructed in $L(\alpha)$.

Such a pair $(\mathcal{R}, \mathfrak{R})$ will be called a double tree representation for $Y$.

Recall that if $\Re=\left(R^{\xi}\right)_{\xi<\omega_{1}}$ is an interpolation family for $R$, then there exists $\gamma<\omega_{1}$ such that $R^{\gamma}=R$, and the value of $\gamma$ depends on the complexity of $Y$. 
Examples 2.9. We leave the checking of the following results to the reader:

1) If $Y$ is a $\Sigma_{1}^{0}$ set, the double tree constructed in Example 2.4 is interpolable with $\gamma=0$.

2) If $Y$ is a $\Sigma_{2}^{0}$ set, the double tree constructed in Example 2.5 is interpolable with $\gamma=0$.

3) With some little work one can derive from Lemma 1 in 5 a construction from a $\Sigma_{3}^{0}$ set of a double tree which is interpolable with $\gamma=1$.

\section{The game $G$}

In this section we introduce a game $G$ and reduce the proof of the Main Theorem to a result about this game.

General setting: First we fix:

- $X$ a $\boldsymbol{\Delta}_{1}^{1}$ subset of $2^{\omega} \times 2^{\omega}, Y$ a $\boldsymbol{\Delta}_{1}^{1}$ subset of $2^{\omega}, Z$ a $\boldsymbol{\Pi}_{1}^{1}$ subset of $2^{\omega}$,

- $T$ a standard tree on $\{0,1\} \times \omega_{1}$ (see [7, p. 84) such that for all $z \in 2^{\omega}$

$$
z \in Z \Longleftrightarrow \exists \bar{\rho} \in \omega_{1}^{\omega}, \quad(z, \bar{\rho}) \in\lceil T\rceil,
$$

- A double tree representation $(\mathcal{R}, \mathfrak{R})$ of $Y$ with $\mathcal{R}=\left(R^{+}, R^{\bullet}\right)$ and $\mathfrak{R}=$ $\left(R^{\xi}\right)_{\xi<\omega_{1}}$,

- $\gamma<\omega_{1}$ such that $R^{\gamma}=R:=R^{+} \circ R^{\bullet}$,

$-\alpha \in \omega^{\omega}$ such that: $T, R^{+}, R^{\bullet}, \mathfrak{R}$ are all in $L(\alpha)$ (see Theorem 2.8),

and we set

$$
\begin{aligned}
& -\Omega=\operatorname{Seq}\left(\omega_{1}\right), \\
& -\Sigma=\operatorname{Seq}(\Omega)=\operatorname{Seq}\left(\operatorname{Seq}\left(\omega_{1}\right)\right) .
\end{aligned}
$$

We endow both spaces $\Omega$ and $\Sigma$ with their canonical lexicographical wellordering (see 2.1). For more clarity we shall reserve the letters $\rho$ and $\theta$ (with various subscripts) to denote elements of $\Omega$ and $\Sigma$ respectively.

Description of the game: We consider the game $G$ in which, alternatively, Player $I$ chooses an element in $\{0,1\}$ and Player II chooses an element either in $\{0,1\}$ or in $\{0,1\} \times \Omega$. A move of Player II in which he chooses an element in $\{0,1\}$ will be called a trivial move.

Thus in a position $u$ in $G$, Player I constructs some $s \in$ Seq (2) and Player II constructs a couple $(t, \tau)$ with $t \in \operatorname{Seq}(2)$ and

$$
\tau: J \rightarrow \Omega
$$

a partial function with domain $J \subset \operatorname{Dom}(t)$ such that:

- At his $k+1^{\text {th }}$ move in $u$ Player $I$ chooses: $s(k) \in\{0,1\}$.

- At his $k+1^{\text {th }}$ move in $u$ Player II chooses

$$
\begin{cases}t(k) \in\{0,1\} & \text { if } k \notin J, \\ (t(k), \tau(k)) \in\{0,1\} \times \Omega & \text { if } k \in J .\end{cases}
$$

We shall identify the position $u$ with the triple

$$
(s, t, \tau) \text {. }
$$

Notice that $\operatorname{Dom}(\tau) \subset \operatorname{Dom}(t)=|t|$, and $|s|=|t|+1$ or $|s|=|t|$ depending whether the last move in $u$ is made by Player $I$ or by Player II .

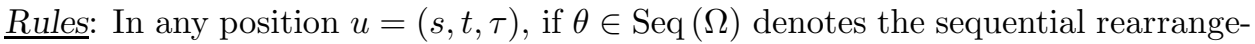
ment of $\tau$ (see 2.1), we require that Player II respects the following two rules:

$\left(\mathrm{R}_{1}\right)$ : For all $k<|\theta|$, if $|\theta(k)|=p$, then $\left(t_{\mid p}, \theta(k)\right) \in T$. 
$\left(\mathrm{R}_{2}\right)$ : The mapping $s_{\left.\right|_{k}} \mapsto \theta(k)$ from $\left(\left\{s_{\left.\right|_{k}} ; k<|\theta|\right\}, R^{+}\right)$to $(\Omega, \preceq)$ is a tree homomorphism.

Notice that $\left(\mathrm{R}_{1}\right)$ and $\left(\mathrm{R}_{2}\right)$ depend only on $\theta$ and the restriction of $s$ to $|\theta|$, and both rules concern only Player II. Thus Player $I$ is totally free in his moves in the game. However, Player II can always extend any position $u$ by playing trivial moves only, so that no new conditions are added in rules $\left(\mathrm{R}_{1}\right)$ and $\left(\mathrm{R}_{2}\right)$ above; such an extension of $u$ will be called a trivial extension.

Runs: It follows from the last remarks that all runs in this game are infinite. We shall identify a run $\bar{u}$ with a triple $(y, z, \tau)$, where $y$ and $z$ are in $2^{\omega}$ and $\tau$ is a partial function from $\omega$ to $\operatorname{Seq}(\Omega)$. We shall refer to $y$ and $z$ as the reals constructed in the run by Player I and Player II respectively. Notice that, unlike $y$ and $z$, which are always infinite, $\tau$ might have a finite domain, which means that from some point on, all Player II 's moves in the run $\bar{u}$ are trivial; and in this case we shall say that $\bar{u}$ is a trivial run.

Win condition: Player II wins the run $(y, z, \tau)$ iff

$$
(y \notin Y) \text { or } \quad((y, z) \in X \text { and } \operatorname{Dom}(\tau) \text { is infinite }) .
$$

We now state the Main Lemma, which we will admit for the moment. The proof of this lemma will be achieved only at the end of Section 6. We shall show here how to derive the Main Theorem from this lemma.

Main Lemma 3.1. Assume that $\aleph_{\gamma+1}^{L(\alpha)}<\aleph_{1}$. For any strategy $\sigma \in L(\alpha)$ for Player $I$ in the game $G$, there exists a compact subset $K$ of $2^{\omega}$ satisfying:

a) Any $y \in K \backslash Y$ can be constructed by Player I in a run compatible with $\sigma$.

b) Any $z \in Z$ can be constructed by Player II in a non-trivial run, compatible with $\sigma$, and in which Player I constructs some real in $K$.

Main Theorem 3.2. Assume that " $\forall \alpha \in \omega^{\omega}, \aleph_{1}^{L(\alpha)}<\aleph_{1}$ ".

Let $X$ and $Y \times Z$ be two subsets of $2^{\omega} \times 2^{\omega}$ with $X$ and $Y$ Borel, and $Z$ coanalytic.

If for any compact subset $K$ of $Y$ there exists $z \in Z$ such that $K \times\{z\} \subset X$, then there exists a continuous mapping $f: 2^{\omega} \rightarrow 2^{\omega}$ such that $f_{\left.\right|_{Y}} \subset X \cap(Y \times Z)$, where $f_{\left.\right|_{Y}}$ denotes the graph of the restriction of $f$ to $Y$.

Proof. Consider the game $G$ above; then clearly $G$ can be represented as a Borel game on $2 \times 2 \times \omega_{1}$, hence determined. More precisely, notice that the win condition for Player II is the conjunction of a Borel condition in the factor $2^{\omega} \times 2^{\omega}$ and a closed condition in the factor $2^{\omega} \times \omega_{1}^{\omega}$; then, since $G$ is definable in $L(\alpha)$, a general absoluteness argument (see Proposition 4.3 in [3]) ensures that the winning player in $G$ has a winning strategy in $L(\alpha)$.

Also notice that there exists $\beta \in \omega^{\omega}$ such that $\aleph_{\gamma+1}^{L(\alpha)}=\aleph_{1}^{L(\beta)}$, which is countable by the assumptions of Theorem 3.2, hence the assumption of Lemma 3.1] is realized.

Claim: Player II is the winner of the game $G$.

Proof. Otherwise, since $G$ is determined, Player $I$ would have a winning strategy $\sigma$, and by the previous observations we can suppose that $\sigma \in L(\alpha)$. Consider then the compact set $K$ given by Lemma 3.1. It follows from the win condition of the game that in any run compatible with $\sigma$, the real constructed by Player $I$ is in $Y$; so a) proves that $K \backslash Y$ is empty - that is, $K$ is a compact subset of $Y$. On the other hand, it also follows from the win condition of the game that in any non-trivial run 
$(y, z, \tau)$ compatible with $\sigma$ we necessarily have $(y, z) \notin X$; so $b)$ proves that for no $z \in Z$ is the set $K \times\{z\}$ contained in $X$, and this contradicts the hypothesis of Theorem [3.2, and proves the claim.

Fix a winning strategy $\sigma^{\prime}$ for Player II; such a strategy clearly defines a mapping $F: y \mapsto(z, \tau)$ such that for all $y \in 2^{\omega}$ the "triple" $(y, F(y))$ is a run compatible with $\sigma^{\prime}$, and whose first coordinate $f: y \mapsto z$ is a continuous (in fact Lipschitz) function from $2^{\omega}$ to $2^{\omega}$

Fix $y \in Y$ and set $F(y)=(z, \tau)$. By the win condition of the game, $(y, z) \in X$ and $\operatorname{Dom}(\tau) \subset \omega$ is infinite; let $\theta \in \Omega^{\omega}$ be the sequential rearrangement of $\tau$. Since $y \in Y$, then by Theorem 2.8 the set $\{s: s \prec y\}$ contains an infinite chain for $R^{+}$; hence by rule $\left(\mathrm{R}_{2}\right)$ we can find an infinite sequence $\left(i_{k}\right)_{k \in \omega}$ such that $\left(\theta\left(i_{k}\right)\right)_{k \in \omega}$ is an infinite chain in $(\Omega, \preceq)$ and defines some $\rho \in \omega_{1}^{\omega}$ such that $\theta\left(i_{k}\right) \prec \rho$ for all $k$. It follows then from rule $\left(\mathrm{R}_{1}\right)$ that $(z, \rho) \in\lceil T\rceil$; hence, by the choice of $T$, that $z \in Z$. This proves that $f_{\left.\right|_{Y}} \subset X \cap(Y \times Z)$.

From now on, $\sigma \in L(\alpha)$ denotes a fixed strategy for Player $I$ in the game $G$.

All runs and positions will implicitly be supposed to be compatible with $\sigma$.

\section{The Double tree analysis of $\sigma$}

4.1. Notation. We denote by $W$ the set of all finite positions in $G$ compatible with $\sigma$ and in which the last move is made by Player II. We shall denote by $\prec$ the relation on $W$ defined by the natural extension of positions in the game. Notice that if $u=(s, t, \tau) \in W$, then $s$ is constructed by Player $I$ before Player II has played his last move in $(t, \tau)$; in other words, $s$ can be viewed as "the answer" by $\sigma$ to $u^{*}$, the predecessor of $u$ in $W$.

For $u=(s, t, \tau) \in W$ we set

$$
\begin{aligned}
& \varphi(u)=(s, t, \operatorname{Dom}(\tau)), \\
& \psi(u)=\text { the sequential rearrangement of the partial function } \tau .
\end{aligned}
$$

Notice that $|s|=|t|$ and $\operatorname{Dom}(\tau) \subset \operatorname{Dom}(t)$; and we can identify $\operatorname{Dom}(\tau)$, via its characteristic function on Dom $(t)$, to an element of Seq (2) (of the same length as $s$ and $t$ ); hence, by a new identification we can, and shall, view $\varphi(u)$ as an element of Seq $(2 \times 2 \times 2)$. One should think of $\varphi(u)$ as the "countable part of the information in $u$ ", in opposition to $\psi(u)$, which lives in a large space. It is clear that the couple $(\varphi(u), \psi(u))$ determines $u$ entirely, so the mapping

$$
(\varphi, \psi): W \rightarrow \operatorname{Seq}(2 \times 2 \times 2) \times \operatorname{Seq}(\Omega)
$$

is one-to-one.

We also denote by $\prec$ the extension relation in both spaces Seq $(2 \times 2 \times 2)$ and $\operatorname{Seq}(\Omega)$. For $u$ and $v$ in $W$ we clearly have

$$
u \prec v \quad \Longleftrightarrow \quad \varphi(u) \prec \varphi(v) \text { and } \psi(u) \preceq \psi(v)
$$

and the particular case where $\varphi(u) \prec \varphi(v)$ and $\psi(u)=\psi(v)$ corresponds to the case where $v$ is a trivial extension of $u$. We shall also for simplicity denote by $|u|$ the height of the element $u$ in the tree $(W, \preceq)$, and call it the length of $u$. It is clear that

We also set

$$
|\psi(u)| \leq|\varphi(u)|=|u|
$$

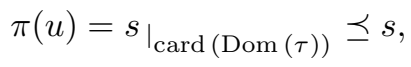


which is the precise part of $s$ needed by Player II to ensure rules $\left(\mathrm{R}_{1}\right)$ and $\left(\mathrm{R}_{2}\right)$; this parameter will play a major role in the proof. Finally, for all $s \in \operatorname{Seq}(2)$, all $u \in W$ and all $\xi<\omega_{1}$ we set

$$
\begin{aligned}
I_{\xi}(s) & =\left\{\begin{array}{ll}
k<|s|: \quad s_{\uparrow_{k}} & R^{\xi} s
\end{array}\right\}, \\
J_{\xi}(u) & =\left\{m \in \operatorname{Dom}(\tau): \pi\left(u_{\uparrow_{m}}\right) R^{\xi} \pi(u)\right\}, \\
\psi_{\xi}(u) & =\text { the sequential rearrangement of the partial function } \tau_{\left.\right|_{J_{\xi}(u)}} .
\end{aligned}
$$

In particular, for $\xi=0$ we have

$$
I_{0}(s)=|s|, \quad J_{0}(u)=\operatorname{Dom}(\tau), \quad \psi_{0}=\psi .
$$

Notice that if

$$
\jmath: \operatorname{card}(\operatorname{Dom}(\tau)) \rightarrow \operatorname{Dom}(\tau)
$$

denotes the unique increasing enumeration of $\operatorname{Dom}(\tau)$, then

$$
J_{\xi}(u)=\jmath\left(I_{\xi}(\pi(u))\right) .
$$

Thus $J_{\xi}(u)$ is just a formal copy of some $I_{\xi}(s)$, and its introduction might appear to the reader as redundant. In fact, in the sequel we shall work with the $J_{\xi}(u)$ 's exclusively until the last lemma (Lemma 6.8), where we will have to consider some $I_{\xi}(s)$ which will appear later as $J_{\xi}(u)$ for some position $u$ that we will construct during the proof.

Lemma 4.2. For any $u \in W$ and any $\xi<\omega_{1}$ we have:

(a) For all $m \in J_{\xi}(u)$ there exists a unique $k<\operatorname{card}\left(J_{\xi}(u)\right)$ such that $\psi_{\xi}\left(u_{{ }_{1 m}}\right)$ $=\psi_{\xi}(u)_{\uparrow_{k}}$.

(b) For all $k<\operatorname{card}\left(J_{\xi}(u)\right)$ there exists a unique $m \in J_{\xi}(u)$ such that $\psi_{\xi}\left(u_{\lceil m}\right)$ $=\psi_{\xi}(u)_{\uparrow_{k}}$.

Proof. Let $\jmath: \operatorname{card}(\operatorname{Dom}(\tau)) \rightarrow \operatorname{Dom}(\tau)$ be as above. It follows from the definition of the game that, for all $k<|\pi(u)|=\operatorname{card}(\operatorname{Dom}(\tau))$, if $m=\jmath(k)$, then $\pi(u)_{\uparrow_{k}}=$ $\pi\left(u_{\uparrow m}\right)$.

The main point in the previous definitions is that both families $\left(I_{\xi}(s)\right)_{\xi<\omega_{1}}$ and $\left(J_{\xi}(u)\right)_{\xi<\omega_{1}}$ reflect, in an obvious way, the properties of the family $\left(R^{\xi}\right)_{\xi<\omega_{1}}$. However, for completeness we state the following two straightforward results, which we will use extensively in the sequel. Notice that in both results, (i) and (ii) express that $R^{\xi}$ is a tree relation: (i) is a reformulation of the reflexivity of $R^{\xi}$, and (ii) states that the initial segment of $s$ is totally ordered. Also, (iii) and (iv) are a reformulation of the fact that the family $\left(R^{\xi}\right)_{\xi<\omega_{1}}$ is decreasing and continuous at limit ordinals. Finally, (v) is a reformulation of the interpolation property.

Lemma 4.3. For any $s \in \operatorname{Seq}(2)$ and any $\xi<\omega_{1}$ we have:

(i) $\max I_{\xi}(s)=|s|-1$.

(ii) If $k \in I_{\xi}(s)$, then $I_{\xi}\left(s_{\uparrow_{k}}\right)=I_{\xi}(s) \cap[0, k]$.

(iii) $I_{\xi+1}(s) \subset I_{\xi}(s)$.

(iv) If $\xi$ is limit, then there exists $\eta<\xi$ such that $I_{\xi}(s)=I_{\eta}(s)$.

(v) If $h<k$ with $h \in I_{\xi+1}(s)$ and $k \in I_{\xi}(s)$, then $h \in I_{\xi+1}\left(s_{\uparrow_{k}}\right)$.

Lemma 4.4. For any $u \in W$ and any $\xi<\omega_{1}$ we have:

(i) $\max J_{\xi}(u)=\max J_{0}(u)$.

(ii) If $m \in J_{\xi}(u)$, then $J_{\xi}\left(u_{\lceil m}\right)=J_{\xi}(u) \cap[0, m]$. 
(iii) $J_{\xi+1}(u) \subset J_{\xi}(u)$.

(iv) If $\xi$ is limit, then there exists $\eta<\xi$ such that $J_{\xi}(u)=J_{\eta}(u)$.

(v) If $m<n$ with $m \in J_{\xi+1}(u)$ and $n \in J_{\xi}(u)$, then $m \in J_{\xi+1}\left(u_{{ }_{i n}}\right)$.

4.5. Characteristic sequence. All clauses below refer to Lemma 4.3,

Fix some $s \in \operatorname{Seq}(2)$. It follows from clauses (iii) and (iv) that the decreasing family $\left(I_{\xi}(s)\right)_{\xi<\omega_{1}}$ of finite sets takes only finitely many values, and there exists a unique decreasing sequence $\left(\xi_{i}\right)_{0 \leq i \leq p}$ in $\omega_{1}$, of maximal length, satisfying

$$
\left\{\begin{array}{l}
\xi_{0}=\gamma \\
\xi_{i}=\max \left\{\xi<\xi_{i-1}: I_{\xi_{i-1}}(s) \subsetneq I_{\xi}(s)\right\} \quad \text { if } i>0
\end{array}\right.
$$

(notice that the max is well defined by (iv)), or, equivalently,

$$
\begin{cases}I_{\xi}(s)=I_{0}(s) & \text { if } 0 \leq \xi \leq \xi_{p} \\ I_{\xi}(s)=I_{\xi_{i}}(s) & \text { if } \xi_{i+1}<\xi \leq \xi_{i} \text { and } i<p .\end{cases}
$$

Now if we set

then by clause (ii) we have

$$
\left\{\begin{array}{l}
\ell=\max I_{0}(s)=|s|-1 \\
k_{i}=\max I_{\xi_{i}}(s) \backslash\{\ell\}
\end{array} \text { for all } i \in[0, p],\right.
$$

$$
I_{\xi_{i}}(s)=I_{\xi_{i}}\left(s_{\uparrow_{k_{i}}}\right) \cup\{\ell\} .
$$

Moreover, for $i>0$, since $k_{i-1} \in I_{\xi_{i-1}}(s)=I_{\xi_{i}+1}(s)$ and $k_{i} \in I_{\xi_{i}}(s)$, then it follows from clause $(\mathrm{v})$ that

$$
k_{i-1} \in I_{\xi_{i}+1}\left(s_{\uparrow_{k_{i}}}\right) .
$$

The sequence $\chi(s)=\left(\xi_{i}, k_{i}\right)_{0 \leq i<p}$ will be called the characteristic sequence of $s$, and we shall denote by $\chi_{0}(s)=\left(\xi_{i}\right)_{0 \leq i \leq p}$ its first coordinate.

Now fix some $u \in W$ and set $s=\pi(u)$. We recall that $\operatorname{card}\left(J_{0}(u)\right)=|s|=$ $I_{0}(s)$, and if $\jmath: I_{0}(s) \rightarrow J_{0}(u)$ is the unique increasing enumeration of $J_{0}(u)$, then $J_{\xi}(u)=\jmath\left(I_{\xi}(s)\right)$ for all $\xi$ (see 4.1 for more details). If $\chi(s)=\left(\xi_{i}, k_{i}\right)_{0 \leq i \leq p}$ is the characteristic sequence of $s$, set, for all $i \in[0, p], m_{i}=\jmath\left(k_{i}\right)$.

The sequence $\tilde{\chi}(u)=\left(\xi_{i}, m_{i}\right)_{0 \leq i \leq p}$ will be called the characteristic sequence of $u$, and we shall also denote by $\tilde{\chi}_{0}(u)=\left(\xi_{i}\right)_{0 \leq i \leq p}$ its first coordinate.

Obviously

$$
\begin{cases}J_{\xi}(u)=J_{0}(u) & \text { if } 0 \leq \xi \leq \xi_{p} \\ J_{\xi}(u)=J_{\xi_{i}}(u) & \text { if } \xi_{i+1}<\xi \leq \xi_{i} \text { and } i<p\end{cases}
$$

and if we set

$$
\left\{\begin{array}{l}
n=\max J_{0}(u)=\operatorname{card}\left(J_{0}(u)\right) \\
m_{i}=\max J_{\xi_{i}}(u) \backslash\{n\}
\end{array} \text { for all } i \in[0, p],\right.
$$

then

and, if $i>0$,

$$
J_{\xi_{i}}(u)=J_{\xi_{i}}\left(u_{\uparrow_{m_{i}}}\right) \cup\{n\}
$$

$$
m_{i-1} \in J_{\xi_{i}+1}\left(u_{\left\lceil m_{i}\right.}\right) .
$$

The notion of characteristic sequence will play a fundamental role in all the sequel. In particular, the last property (more precisely, the corresponding property for $\chi(s)$ ) will be crucial for the proof of Lemma 6.8. Notice that, as it appears from the previous observations, this property follows from clause (v) of Lemma 
4.3, which itself is the reformulation of the interpolabilty property of the family $\left(R^{\xi}\right)_{\xi<\omega_{1}}$.

4.6. Trivial modification. We now introduce a technical notion which will play a crucial role in the sequel.

Let $\tau^{\prime}: J^{\prime} \rightarrow \Omega$ and $\tau^{\prime \prime}: J^{\prime \prime} \rightarrow \Omega$ be two partial functions. We shall say that $\tau^{\prime \prime}$ is a trivial modification of $\tau^{\prime}$ if

$$
\left\{\begin{array}{c}
J^{\prime}=J \cup\left\{j^{\prime}\right\} \quad \text { and } \quad J^{\prime \prime}=J \cup\left\{j^{\prime \prime}\right\} \quad \text { with } \max (J)<j^{\prime} \leq j^{\prime \prime} \\
\left.\tau^{\prime}\right|_{J}=\left.\tau^{\prime \prime}\right|_{J} \text { and } \quad \tau^{\prime}\left(j^{\prime}\right)=\tau^{\prime \prime}\left(j^{\prime \prime}\right) .
\end{array}\right.
$$

If $u^{\prime}=\left(s^{\prime}, t^{\prime}, \tau^{\prime}\right)$ and $u^{\prime \prime}=\left(s^{\prime \prime}, t^{\prime \prime}, \tau^{\prime \prime}\right)$ are two positions in the game, we shall say that $u^{\prime \prime}$ is a trivial modification of $u^{\prime}$ if

$$
t^{\prime} \preceq t^{\prime \prime} \quad \text { and } \quad \tau^{\prime \prime} \text { is a trivial modification of } \tau^{\prime} \text {. }
$$

Notice that since $s^{\prime}$ is constructed by Player I before Player II's last move, then we necessarily have

$$
s^{\prime} \preceq s^{\prime \prime} .
$$

This notion is quite close to - but more elaborate than - the notion of trivial extension introduced formerly, which corresponds to the particular case where $J^{\prime}=$ $J^{\prime \prime}$ (hence $\tau^{\prime}=\tau^{\prime \prime}$ ). In fact, if we assume for simplicity that in both positions $u^{\prime}$ and $u^{\prime \prime}$ the last moves are non-trivial, then saying that $u^{\prime \prime}$ is a trivial modification of $u^{\prime}$ means that if $u$ denotes the predecessor of $u^{\prime}$ in $W$, then

1) $u^{\prime \prime}$ is a trivial extension of $u$ (in particular, $u \prec u^{\prime \prime}$ ), and

2) the ordinal chosen by Player II in his last moves in $u^{\prime}$ and $u^{\prime \prime}$ is the same.

Finally, we mention that for the proof of the main result below we will need to define a number of operations on some sets of positions, and one of the key arguments at the end will rely on the fact that all that these sets are invariant under trivial modifications, and all these operations are "compatible" with trivial modifications.

\section{Main CONSTRUCtion}

The proof of the Main Lemma in the next section will rely on an inductive construction that we detail in the present section. To give a synthetic view of this construction we summarize the results of this section, and in particular all properties needed for the next section, through the following theorem.

Theorem 5.1. There exist decreasing families $\left(V_{\xi}\right)_{\xi<\omega}$ of subsets of $W,\left(\tilde{E}_{\xi}\right)_{\xi<\omega}$ of equivalence relations on $W$, and $\left(E_{\xi}\right)_{\xi<\omega}$ of equivalence relations on $\Sigma$ satisfying the following conditions:

(1) $V:=V_{\gamma}=V_{\xi}$, for all $\xi \geq \gamma$.

(2) $E_{0}=\Sigma \times \Sigma$ has only one class, and $E_{1+\xi}$ has at most $\aleph_{\xi+1}^{L(\alpha)}$ classes.

(3) $u \tilde{E}_{\xi} v \Longleftrightarrow\left(\varphi(u)=\varphi(v)\right.$ and $\left.\forall \eta \leq \xi, \psi_{\eta}(u) E_{\eta} \psi_{\eta}(v)\right)$.

(4) If $u, v \in V_{\xi}$ with $u \tilde{E}_{\xi} v$ and $\psi_{\xi}(u)=\psi_{\xi}(v)$, then $u=v$.

(5) If $u \in V_{\xi}$ and $m \in J_{\xi}(u)$, then $u_{\lceil m} \in V_{\xi}$. If $u, v \in W$ with $u \tilde{E}_{\xi} v$ and $m \in J_{\xi}(u)$, then $u_{{ }_{\uparrow m}} \tilde{E}_{\xi} v_{\uparrow_{m}}$.

(6) If $u, v \in V_{\xi}$ with $u \tilde{E}_{\xi} v$, then for any $\eta<\xi$ such that $J_{\eta+1}(u)=J_{\xi}(u)$ and any $u^{\prime} \in V_{\xi}$ extending $u$ with $u=u^{\prime}{ }_{{ }_{1 m}}$ for some $m \in J_{\xi}\left(u^{\prime}\right)$, there exists $v^{\prime} \in V_{\xi}$ extending $v$ and such that $u^{\prime} \tilde{E}_{\eta} v^{\prime}$. 
(7) There exists a partial function $\nu$ from $W$ to $V$ which, to any position $w \in W$ with $\tilde{\chi}(w)=\left(\xi_{i}, m_{i}\right)_{0 \leq i \leq p}$ and satisfying $w_{{ }_{i m_{i}}} \in V_{\xi_{i}}$ for all $i \in[0, p]$, assigns a position $\nu(w) \in V$ such that $\nu(w) \tilde{E} w$ and $\psi_{\gamma}(\nu(w))=\psi_{\gamma}(w)$.

Moreover, if $w^{\prime}$ is a trivial modification of $w$, then $\nu\left(w^{\prime}\right)$ is a trivial modification of $\nu(w)$.

We recall that we fixed (see Section 3) some $\alpha \in \omega^{\omega}$ and some ordinal $\gamma<\omega_{1}$.

To prove the theorem we shall explicitly define the families $\left(V_{\xi}\right),\left(\tilde{E}_{\xi}\right),\left(E_{\xi}\right)$, and then prove, through a list of lemmas, their main properties. We leave it to the reader to check that all the statements of Theorem $5.1 \mathrm{can}$ be extracted from these lemmas.

- Notice that $\tilde{E}_{0}$ is just the canonical equivalence relation associated to the function $\varphi$.

- By property (3) the equivalence relation $\tilde{E}_{\xi}$ on $W$ is reducible to the product of a simple relation $\tilde{E}_{0}$ on $W$ and a family $\left(E_{\eta}\right)_{\eta \leq \xi}$ of equivalence relations on $\Sigma$, and the equivalence $u \tilde{E}_{\xi} v$ can be obtained by ensuring that $\varphi(u)=\varphi(v)$ and separately by some control on the $\psi_{\eta}(u)$ 's and the $\psi_{\eta}(v)$ 's for $\eta \leq \xi$.

- Roughly speaking, the equivalence $\theta E_{\xi} \theta^{\prime}$ will mean $\theta$ and $\theta^{\prime}$ are indiscernable of order $\xi$ by the strategy $\sigma$, in the sense that for any positions $u$ and $u^{\prime}$ such that $\psi_{\xi}(u)=\theta$ and $\psi_{\xi}\left(u^{\prime}\right)=\theta^{\prime}$ the strategy reacts similarly in a lot of other positions related to $u$ and $u^{\prime}$.

$-V_{\xi}$ is a family of positions $u$ for which the indiscernability of order $\xi$ (of their respective $\psi_{\xi}(u)$ ) behaves nicely towards the extension relation, as formulated in property $(6)$.

- In fact we would have liked to ensure that $V_{\xi}$ is a subtree of $W$ (for the extension relation of positions) and that $\tilde{E}_{\xi}$ is compatible with the restriction operation; but this is not the case. However, (5) asserts some hereditarity of $V_{\xi}$ with respect to the sets $J_{\xi}(u)$, which appear as sets of levels where properties of "order $\xi$ " are preserved by truncation. This last idea is a fundamental ingredient in the construction as well as the proof of the Main Lemma in the next section. This also explains the high occurence of the quantifiers " $\forall m \in J_{\xi}(u) \ldots$ " and " $\exists m \in J_{\xi}(u) \ldots$ " in the coming definitions.

- We also would have liked to ensure that any position $w$ in $W$ is $\tilde{E}$-equivalent to some position $v$ in $V$. This is not possible, but property (7) asserts that we can manage this if enough restrictions of $w$ are already in $V$. Notice that such a $v$ is not unique, and the $\nu$ operator provides only a particular way of finding $v$. The last part of $(7)$ means that the computation of $\psi(\nu(w))$ does not depend on the time at which the last non-trivial move is played in $w$; this precision is very important for the sequel.

We now proceed to the definition of the three families $\left(V_{\xi}\right),\left(\tilde{E}_{\xi}\right),\left(E_{\xi}\right)$, which will involve in fact two other families $\left(\tilde{\Phi}_{\xi}\right),\left(\Phi_{\xi}\right)$ of functions on $W$ and $\Sigma$ respectively.

But first we fix some notation. If $\left(A_{i}\right)_{i \in I}$ is a family of sets, we shall denote its disjoint union $\bigcup\left\{\{i\} \times A_{i} ; i \in I\right\}$ by $\dot{\bigcup}\left\{\{i\} \times A_{i} ; i \in I\right\}$ or by $\bigcup_{i \in I} A_{i}$. We shall also denote by $A \dot{\cup} B$ the disjoint union of the family $(A, B)$. 
Definition 5.2. We define inductively for all $\xi<\omega_{1}$ :

(i) $V_{0}=W$,

$$
\begin{aligned}
& V_{\lambda}=\bigcap_{\eta<\lambda} V_{\eta} \quad \text { if } \lambda \text { is limit, } \\
& V_{\eta+1}=\left\{u \in V_{\eta} \quad: \quad \forall m \in J_{\eta+1}(u), \quad \psi_{\eta}\left(u_{\lceil m}\right)=\min \left\{\psi_{\eta}\left(u^{\prime}\right) ; u^{\prime} \in\right.\right. \\
& \left.V_{\eta}\left(u_{\lceil m}\right)\right\}, \\
& \text { where for all } v \in W \\
& \qquad V_{\eta}(v)=\left\{v^{\prime} \in V_{\eta}: v^{\prime} \tilde{E}_{\eta} v, \psi_{\eta+1}\left(v^{\prime}\right)=\psi_{\eta+1}(v)\right\} .
\end{aligned}
$$

$$
\begin{aligned}
& \Phi_{\xi}(\theta)=\bigcup_{\substack{k, m, \eta) \\
\in|\theta| \times \omega \times \xi}}^{\bullet}\left\{\tilde{\Phi}_{\eta}(u) ; u \in V_{\eta+1}, m \in J_{\eta+1}(u), \text { and } \psi_{\eta+1}\left(u_{{ }_{1 m}}\right)=\right. \\
& \left.\theta_{\uparrow_{k}}\right\}, \\
& \theta E_{\xi} \theta^{\prime} \Longleftrightarrow \Phi_{\xi}(\theta)=\Phi_{\xi}\left(\theta^{\prime}\right) .
\end{aligned}
$$

(iii) $\tilde{\Phi}_{\xi}(u)=\varphi(u) \dot{\cup} \bigcup_{0<\eta \leq \xi} \Phi_{\eta}\left(\psi_{\eta}(u)\right)$,

$$
u \tilde{E}_{\xi} u^{\prime} \Longleftrightarrow \tilde{\Phi}_{\xi}(u)=\tilde{\Phi}_{\xi}\left(u^{\prime}\right) .
$$

This definition is clearly well-founded: First notice that $V_{0}=W$ and $\Phi_{0}$ is the constant 0-valued function; and assuming that $V_{\eta}, \Phi_{\eta}$ are defined for all $\eta<\xi$, then $V_{\xi}, \Phi_{\xi}, E_{\xi}, \tilde{\Phi}_{\xi}, \tilde{E}_{\xi}$ are successively defined by (i), (ii) and (iii). In particular $E_{0}=\Sigma \times \Sigma$ is the trivial relation, and $\tilde{E}_{0}$ is the canonical relation associated to $\tilde{\Phi}_{0}=\varphi$ :

$$
u \tilde{E}_{0} u^{\prime} \Longleftrightarrow \varphi(u)=\varphi\left(u^{\prime}\right) .
$$

For the convenience of the induction in the previous definition as well as in all the coming proofs, we allowed the parameter $\xi$ to be an arbitrary countable ordinal. But in fact we shall deal later only with the families $\left(V_{\xi}\right)_{\xi \leq \gamma},\left(\tilde{E}_{\xi}\right)_{\xi \leq \gamma},\left(E_{\xi}\right)_{\xi \leq \gamma}$. More particularly, the last element of each of these families will play a central role, and we shall set

$$
V=V_{\gamma}, \quad \tilde{E}=\tilde{E}_{\gamma}, \quad E=E_{\gamma} .
$$

Lemma 5.3. The families $\left(V_{\xi}\right)_{\xi<\omega_{1}},\left(\tilde{E}_{\xi}\right)_{\xi<\omega_{1}},\left(E_{\xi}\right)_{\xi<\omega_{1}}$ are decreasing. Moreover, $V_{\xi}=V$ for all $\xi \geq \gamma$.

Proof. The first part of the lemma follows directly from the definitions. For the second part, notice that if $\xi \geq \gamma$, then $J_{\xi}(u)=J_{\gamma}(u)$ and $\psi_{\xi}(u)=\psi_{\gamma}(u)$ for all $u$; hence $V_{\xi}=V_{\gamma}$.

Lemma 5.4. Let $\xi<\omega_{1}$.

a) If $\theta E_{\xi} \theta^{\prime}$ and $k<|\theta|$, then $\theta_{\uparrow_{k}} E_{\xi} \theta^{\prime} \uparrow_{k}$.

b) If $u \tilde{E}_{\xi} v$ and $m \in J_{\xi}(u)$, then $u_{\lceil m} \tilde{E}_{\xi} v_{\lceil m}$.

c) If $u \in V_{\xi}$ and $m \in J_{\xi}(u)$, then $u_{\lceil m} \in V_{\xi}$.

d) For all $\eta<\xi$, if $u \in V_{\eta}$ and $J_{\eta}(u)=J_{\xi}(u)$, then $u \in V_{\xi}$.

Proof. a) follows directly from the definitions.

b) Suppose that $u \tilde{E}_{\xi} v$ and let $m \in J_{\xi}(u)$; since $u \tilde{E}_{0} v$, then for all $\eta \leq \xi$ we have $m \in J_{\xi}(u)=J_{\xi}(v) \subset J_{\eta}(u)=J_{\eta}(v)$. Hence by Lemma 4.4 (iii) there exists some $k \leq\left|\psi_{\eta}(u)\right|$ such that $\psi_{\eta}\left(u_{\uparrow_{m}}\right)=\psi_{\eta}(u)_{\uparrow_{k}}$ and $\psi_{\eta}\left(v_{\uparrow_{m}}\right)=\psi_{\eta}(v)_{\uparrow_{k}}$. Now the conclusion follows from a). 
We shall prove $c$ ) and $d$ ) separately by induction on $\xi$. Notice that both statements are obvious for $\xi=0$; and assume that they are satisfied for all $\xi^{\prime}<\xi$.

c) Let $u \in V_{\xi}$ and $m \in J_{\xi}(u)$.

- If $\xi$ is limit, consider any $\eta<\xi$; since $J_{\xi}(u) \subset J_{\eta}(u)$, then by the induction hypothesis we have $u_{\uparrow_{m}} \in V_{\eta}$. This proves that $u_{\uparrow_{m}} \in V_{\xi}$.

- If $\xi=\eta+1$ is a successor, again since $J_{\xi}(u) \subset J_{\eta}(u)$, then by the induction hypothesis we have $v:=u_{\lceil m} \in V_{\eta}$; and since by Lemma 4.4 (iii), $J_{\xi}(v) \subset J_{\xi}(u)$, then for all $p \in J_{\xi}(v)$ we have $\psi_{\eta}\left(v_{\mid p}\right)=\psi_{\eta}\left(u_{\mid p}\right)=\min \left\{\psi_{\eta}\left(v^{\prime}\right) ; v^{\prime} \in V_{\eta}\left(u_{\mid p}\right)=\right.$ $\left.V_{\eta}\left(v_{\mid p}\right)\right\}$ since $v \in V_{\eta}$; this shows that $v \in V_{\xi}$.

d) Let $u \in V_{\eta}$ and $\eta<\xi$ be such that $J_{\eta}(u)=J_{\xi}(u)$.

- If $\xi$ is limit, then for all $\xi^{\prime}$ such that $\eta<\xi^{\prime}<\xi$ we also have $J_{\eta}(u)=J_{\xi^{\prime}}(u)$. Hence by the induction hypothesis $u \in V_{\xi^{\prime}}$; this proves that $u \in V_{\xi}$.

- If $\xi=\xi^{\prime}+1$, then, as in the previous case, we have $u \in V_{\xi^{\prime}}$; we also have $J_{\xi^{\prime}}(u)=J_{\xi}(u)$, whence again by Lemma 4.4 (iii), for any $m \in J_{\xi^{\prime}+1}(u)$ we have $J_{\xi^{\prime}}\left(u_{\uparrow_{m}}\right)=J_{\xi}\left(u_{{ }_{\uparrow m}}\right)$. It follows that for any $v$ which is $\tilde{E}_{0}$-equivalent to $u_{{ }_{\uparrow m}}$ we also have $J_{\xi^{\prime}}(v)=J_{\xi}(v)$; in particular for any $v \in V_{\xi^{\prime}}\left(u_{{ }_{1 m}}\right)$ we have $\psi_{\xi}(v)=\psi_{\xi^{\prime}}(v)=$ $\psi_{\xi^{\prime}}\left(u_{{ }_{1 m}}\right)=\psi_{\xi}\left(u_{\lceil m}\right)$, and obviously $\psi_{\xi}\left(u_{\lceil m}\right)=\min \left\{\psi_{\xi}(v) ; v \in V_{\xi^{\prime}}\left(u_{\lceil m}\right)\right\}$; so $u \in V_{\xi}$.

Lemma 5.5. Let $u, v \in V_{\xi}$ be such that $\psi_{\xi}(u)=\psi_{\xi}(v)$.

a) If $u \tilde{E}_{\eta} v$ for some $\eta<\xi$ such that $J_{\eta+1}(u)=J_{\xi}(u)$, then $u=v$.

b) If $u \tilde{E}_{\xi} v$, then $u=v$.

Proof. We shall prove "a) and b)" by induction on $\xi$. For $\xi=0$, a) is obvious and b) holds since $u \tilde{E}_{0} v \Longleftrightarrow \varphi(u)=\varphi(v)$, and the mapping $(\varphi, \psi)$ is one-to-one. So let $\xi>0$ and assume that a) and b) hold for all $\xi^{\prime}<\xi$; we shall then prove a) and b) for $\xi$.

a) Notice that $u, v \in V_{\xi} \subset V_{\eta+1} \subset V_{\eta}$; moreover, since $u \tilde{E}_{0} v$, then $v$ also satisfies $J_{\eta+1}(v)=J_{\xi}(v)$, so $\psi_{\eta+1}(v)=\psi_{\xi}(v)=\psi_{\xi}(u)=\psi_{\eta+1}(u)$, and $v \in V_{\eta}(u)$ (see $5.2(i)$ ). Then by definition of $V_{\eta+1}$ we have $\psi_{\eta}(u) \leq \psi_{\eta}(v)$, and so by symmetry $\psi_{\eta}(u)=\psi_{\eta}(v)$; and since $u \tilde{E}_{\eta} v$, then the conclusion follows from $b$ ) at level $\eta$.

b) We can assume that $\xi>0$; by Lemma 4.4 (iv) the set $\left\{\zeta<\xi: J_{\zeta}(w) \subsetneq\right.$ $\left.J_{\xi}(w)\right\}$ admits a maximum element $\eta<\xi$, and clearly $J_{\eta+1}(u)=J_{\xi}(u)$; and since $\tilde{E}_{\xi} \subset \tilde{E}_{\eta}$, then the conclusion follows from a) at level $\xi$.

We now are ready to prove statement (6) of Theorem[5.1:

Lemma 5.6. Let $u, v \in V_{\xi}$ and $\eta<\xi$ be such that $u \tilde{E}_{\xi} v$ and $J_{\eta+1}(u)=J_{\xi}(u)$. For any $u^{\prime} \in V_{\xi}$ extending $u$ with $u=u^{\prime}{ }_{i m}$ for some $m \in J_{\xi}\left(u^{\prime}\right)$, there exists $v^{\prime} \in V_{\xi}$ extending $v$ and such that $u^{\prime} \tilde{E}_{\eta} v^{\prime}$.

Proof. Notice first that since $u \tilde{E}_{0} v$, then $J_{\eta+1}(v)=J_{\xi}(v)$. For all $\theta \in \Sigma$ set

$$
\Psi(\theta):=\left\{\tilde{\Phi}_{\eta}(w) ; w \in V_{\eta+1} \text { and } m \in J_{\eta+1}(w), \quad \psi_{\eta+1}\left(w_{\uparrow m}\right)=\theta\right\}
$$

Since $\psi_{\xi}(u) E_{\xi} \psi_{\xi}(v)$, then in particular $\Psi\left(\psi_{\xi}(u)\right)=\Psi\left(\psi_{\xi}(v)\right)$; and by the hypothesis $\tilde{\Phi}_{\eta}\left(u^{\prime}\right) \in \Psi\left(\psi_{\xi}(u)\right)$. Hence $\tilde{\Phi}_{\eta}\left(u^{\prime}\right) \in \Psi\left(\psi_{\xi}(v)\right)$ and $\tilde{\Phi}_{\eta}\left(u^{\prime}\right)=\tilde{\Phi}_{\eta}\left(v^{\prime}\right)$ for some $v^{\prime} \in V_{\eta+1}$ with $\psi_{\eta+1}\left(v^{\prime}{ }_{i m}\right)=\psi_{\xi}(v)$. Then from the very definition of $\tilde{E}_{\eta}$ we have $u^{\prime} \tilde{E}_{\eta} v^{\prime}$; in particular, $u^{\prime} \tilde{E}_{0} v^{\prime}$, and it follows from Lemma 4.2 that $J_{\eta+1}\left(v^{\prime}{ }_{{ }_{m}}\right)=J_{\xi}\left(v^{\prime}{ }_{{ }_{m}}\right)=J_{\xi}(v)$ and $m \in J_{\xi}\left(v^{\prime}\right)=J_{\xi}\left(u^{\prime}\right) \subset J_{\eta+1}\left(u^{\prime}\right)=J_{\eta+1}\left(v^{\prime}\right)$. Then by Lemma 5.4 we also have $v^{\prime}{ }_{{ }_{1 m}} \in V_{\xi}$ and $v^{\prime}{ }_{{ }_{1 m}} \tilde{E}_{\eta} u_{{ }_{i m}}^{\prime}=u \tilde{E}_{\xi} v$; hence 
$v^{\prime}{ }_{{ }_{m} m} \tilde{E}_{\eta} v$. But since $\psi_{\xi}\left(v^{\prime}{ }_{{ }_{m}}\right)=\psi_{\xi}(v)$, it follows then from Lemma 5.5] a) that $v^{\prime}{ }_{i m}=v$; hence $v \preceq v^{\prime}$.

Lemma 5.7. Let $u \in V_{\eta}$, and set $n=\max J_{\eta+1}(u)$ and $m=\max J_{\eta+1}(u) \backslash\{n\}$. If $u_{\uparrow m} \in V_{\eta+1}$, then there exists a unique position $v \in V_{\eta+1}$ such that $\psi_{\eta+1}(v)=$ $\psi_{\eta+1}(u)$ and $v_{\uparrow m}=u_{\uparrow m}$.

Proof. The uniqueness follows from Lemma 5.5, Let $v$ be the unique element of $V_{\eta}(u)$ at which $\psi_{\eta}$ attains its minimum on $V_{\eta}(u)$.

Notice that since $m \in J_{\eta+1}(u) \subset J_{\eta}(u)$, then by Lemma 4.2 there exists a unique $k$ such that $\psi_{\eta}\left(u_{\uparrow_{m}}\right)=\psi_{\eta}(u)_{\uparrow_{k}}$; and since $v \tilde{E}_{0} u$, then the same relation (with the same $k$ ) holds for $v$.

Since $u \in V_{\eta}(u)$, then by the minimal choice of $v$ we have $\psi_{\eta}(v) \leq \psi_{\eta}(u)$; hence by lexicographical ordering we also have $\psi_{\eta}\left(v_{{ }_{\uparrow m}}\right)=\psi_{\eta}(v)_{\uparrow_{k}} \leq \psi_{\eta}(u)_{\uparrow_{k}}=\psi_{\eta}\left(u_{\uparrow_{m}}\right)$. On the other hand, since $m \in J_{\eta+1}(v)=J_{\eta+1}(u)$, then $\psi_{\eta+1}\left(v_{\lceil m}\right)=\psi_{\eta+1}\left(u_{\lceil m}\right)$ and by Lemma $5.4 \mathrm{~b}$ ) and c), $v_{{ }_{1 m}} \in V_{\eta}$ and $v_{\uparrow m} \tilde{E}_{\eta} u_{\uparrow m}$; this proves that $v_{{ }_{1 m}} \in$ $V_{\eta}\left(u_{\lceil m}\right)$. But by the assumptions of the lemma $u_{{ }_{1 m}} \in V_{\eta+1}$; hence $\psi_{\eta}\left(u_{{ }_{1 m}}\right) \leq$ $\psi_{\eta}\left(v_{\lceil m}\right)$. Thus $u_{\uparrow m}$ and $v_{\lceil m}$ are in $V_{\eta}$ with $u_{\lceil m} \tilde{E}_{\eta} v_{{ }_{\uparrow m}}$ and $\psi_{\eta}\left(u_{\lceil m}\right)=\psi_{\eta}\left(v_{\uparrow m}\right)$; so by Lemma $5.5 \mathrm{~b}), u_{\uparrow m}=v_{\uparrow m}$.

Finally, to prove that $v \in V_{\eta+1}$ we have to check that for all $p \in J_{\eta+1}(v)=$ $J_{\eta+1}(u)$ the equality $\psi_{\eta}\left(v_{\uparrow p}\right)=\min \left\{\psi_{\eta}(w) ; w \in V_{\eta}\left(v_{\lceil p}\right)\right\}$ holds.

- If $p=n$, this follows from the choice of $v$.

- If $p \leq m$, then $v_{\uparrow p}=\left(v_{\uparrow m}\right)_{\lceil p}=\left(u_{\uparrow m}\right)_{\lceil p}$, and the conclusion follows from the assumption that $u_{\lceil m} \in V_{\eta+1}$.

Lemma 5.8. There exists a partial function $\nu$ from $W$ to $V$ which, to any position $w \in W$ with $\tilde{\chi}(w)=\left(\xi_{i}, m_{i}\right)_{0 \leq i \leq p}$ and satisfying $w_{{ }_{m_{i}}} \in V_{\xi_{i}}$ for all $i \leq p$, assigns a position $\nu(w) \in V$ such that

a) $\psi_{\gamma}(\nu(w))=\psi_{\gamma}(w), \nu(w)_{\left\lceil m_{0}\right.}=w_{\left\lceil m_{0}\right.}$, and $\nu(w)_{\left\lceil m_{j}\right.} \tilde{E}_{\xi_{j}} w_{\left\lceil m_{j}\right.}$ if $j>0$, and

b) if $w^{\prime}$ is a trivial modification of $w$, then $\nu\left(w^{\prime}\right)$ is a trivial modification of $\nu(w)$.

Proof. a) We shall first construct, by a downward induction, a sequence $\left(w^{(i)}\right)_{0 \leq i \leq p}$ satisfying, for all $i$,

$v^{(i)} \in V_{\xi_{i}}, v^{(i)}{ }_{\left\lceil m_{i}\right.}=w_{\left\lceil m_{i}\right.}, \psi_{\xi_{i}}\left(v^{(i)}\right)=\psi_{\xi_{i}}(w)$, and $v^{(i)} \tilde{E}_{\xi_{i+1}} v^{(i+1)}$ if $i<p$

For the initial value $i=p$ take $v^{(p)}=w$. Since $w \in V_{0}$ and $J_{0}(w)=J_{\xi_{p}}(w)$, then by Lemma $5.4 \mathrm{~d}), v^{(p)} \in V_{\xi_{p}}$; the other clauses are obviously satisfied.

Assume that $v^{(i+1)}$ is constructed. Set $u=v^{(i+1)}, \eta=\xi_{i+1}, m=m_{i}$, so that $u \in V_{\eta}$ and $m \in J_{\eta+1}(u)=J_{\xi_{i}}(u)$; then, applying Lemma 5.7 we get a position $v^{(i)} \in V_{\eta+1}$ such that $v^{(i)} \tilde{E}_{\eta} u, \psi_{\eta+1}\left(v^{(i)}\right)=\psi_{\eta+1}(u)$, and $v^{(i)}{ }_{{ }_{\lceil m}}=u_{{ }_{\uparrow m}}$. So $v^{(i)} \tilde{E}_{\xi_{i+1}} v^{(i+1)}$, and since $J_{\eta+1}\left(v^{(i)}\right)=J_{\xi_{i}}\left(v^{(i)}\right)$, then by Lemma 5.4 $v^{(i)} \in V_{\xi_{i}}$. Moreover, by the induction hypothesis $\psi_{\eta}(u)=\psi_{\eta}(w)$, so, since $u \tilde{E}_{0} w$, then $\psi_{\eta+1}\left(v^{(i)}\right)=\psi_{\eta+1}(w)$. Also, by the induction hypothesis $u_{\left\lceil m_{i+1}\right.}=w_{\left\lceil m_{i+1}\right.}$; hence $v^{(i)}{ }_{\lceil m}=u_{{ }_{\uparrow m}}=\left(u_{\left.\uparrow_{i+1}\right)}\right)_{\lceil m}=w_{{ }_{\uparrow m}}$.

This finishes the inductive construction of the sequence $\left(v^{(i)}\right)_{0 \leq i \leq p}$. Claim: $v^{(i)} \tilde{E}_{\xi_{j}} v^{(j)}$ for all $i \leq j$. 
Proof. Again we argue by downward induction on $i$. This is obvious for $i=j$; so assume that $v^{(i)} \tilde{E}_{\xi_{j}} v^{(j)}$. By construction we have $v^{(i-1)} \tilde{E}_{\xi_{i}} v^{(i)}$, and since $i<j$, then by the monotony of the family $\left(\tilde{E}_{\xi}\right)$ we also have $v^{(i-1)} \tilde{E}_{\xi_{j}} v^{(i)}$; hence $v^{(i-1)} \tilde{E}_{\xi_{j}} v^{(j)}$.

To finish the proof of the lemma, set $\nu(w)=v^{(0)}$. By construction we have $\nu(w) \in V_{\xi_{0}}=V, \psi_{\gamma}(\nu(w))=\psi_{\gamma}(w)$, and $\nu(w)_{\uparrow_{m_{0}}}=w_{\uparrow_{m_{0}}}$. Moreover, for all $j>0$, since $m_{j} \in J_{\xi_{j}}(\nu(w))$, it follows from the claim that $\nu(w)_{{ }_{\left\lceil m_{j}\right.}} \tilde{E}_{\xi_{j}} v^{(j)}{ }_{{ }_{i m_{j}}}=$ $w_{\uparrow m_{j}}$

b) Let $w \in W$ and $\left(v^{(i)}\right)_{0 \leq i \leq p}$ be, as above, the sequence defining $\nu(w)$; let $w^{\prime}$ be a trivial modification of $w$, and let $\left(v^{\prime(i)}\right)_{0 \leq i \leq p}$ be the corresponding sequence defining $\nu\left(w^{\prime}\right)$.

By the proof of Lemma 5.7, for all $i, v^{(i)}$ is the unique element of $V_{\xi_{i+1}}\left(v^{(i+1)}\right)$ at which $\psi_{\xi_{i+1}}$ attains its minimum. From this observation one can easily prove, by a straightforward downward induction, that for all $i, v^{(i)}$ is a trivial modification of $v^{(i)}$; in particular, $\nu\left(w^{\prime}\right)=v^{(0)}$ is a trivial modification of $\nu(w)=v^{(0)}$.

Lemma 5.9. Each of the equivalence relations $E_{1+\xi}$ and $\tilde{E}_{1+\xi}$ has at most $\aleph_{\xi+1}^{L(\alpha)}$ classes.

Proof. We shall use the following elementary fact in the proof: if $I$ is infinite, then

$$
\operatorname{card}\left(\bigcup_{i \in I}^{\bullet} A_{i}\right) \leq \max \left(\operatorname{card}(I), \sup \left\{\operatorname{card}\left(A_{i}\right) ; i \in I\right\}\right)
$$

For the purpose of this proof it will be convenient to allow the value $\xi=-1$. So for all $\xi \geq-1$ let $\Lambda_{\xi}$ and $\widetilde{\Lambda}_{\xi}$ denote the ranges of the functions $\Phi_{1+\xi}$ and $\tilde{\Phi}_{1+\xi}$. Since $\operatorname{card}\left(\Sigma / E_{1+\xi}\right)=\operatorname{card}\left(\Lambda_{\xi}\right)$ and $\operatorname{card}\left(W / \tilde{E}_{1+\xi}\right)=\operatorname{card}\left(\tilde{\Lambda}_{\xi}\right)$, the lemma will follow from the next claim.

Claim: $\max \left(\operatorname{card}\left(\Lambda_{\xi}\right), \operatorname{card}\left(\tilde{\Lambda}_{\xi}\right)\right) \leq \aleph_{\xi+1}^{L(\alpha)}$.

Proof. Notice that since $\Phi_{1+\xi}$ and $\tilde{\Phi}_{1+\xi}$ are definable in $L(\alpha)$ and $\Sigma$ is in $L$, then $\Lambda_{\xi}$ and $\tilde{\Lambda}_{\xi}$ are also in $L(\alpha)$. In fact we shall prove that

$$
L(\alpha) \models \max \left(\operatorname{card}\left(\Lambda_{\xi}\right), \operatorname{card}\left(\tilde{\Lambda}_{\xi}\right)\right) \leq \aleph_{\xi+1},
$$

which will ensure the claim. Thus in what follows, all definitions and computations are made in $L(\alpha)$.

The proof is by induction on $\xi \geq-1$. This is true for $\xi=-1$, since $\Lambda_{0}=\{0\}$ and $\tilde{\Lambda}_{0}=\operatorname{Seq}(2 \times 2 \times 2)$ are countable in $L$. So assume that the inequality holds for all $\eta<\xi$.

a) Set $\tilde{\Lambda}_{\xi}^{\prime}:=\dot{\bigcup}\left\{\{k\} \times \tilde{\Lambda}_{\eta} ; \quad(k, \eta) \in(|\theta|+1) \times(\{-1\} \cup \xi)\right\}$, so that for all $\theta \in \Sigma$ we have $\Phi_{1+\xi}(\theta) \subset \tilde{\Lambda}_{\xi}^{\prime}$, and $\Lambda_{\xi} \subset \mathcal{P}\left(\tilde{\Lambda}_{\xi}^{\prime}\right)$. By the induction hypothesis, for all $\eta<\xi$ we have $\operatorname{card}\left(\tilde{\Lambda}_{\eta}\right) \leq \aleph_{\eta+1} \leq \aleph_{\xi}$; hence $\operatorname{card}\left(\tilde{\Lambda}_{\xi}^{\prime}\right) \leq \aleph_{\xi}$ and $\operatorname{card}\left(\Lambda_{\xi}\right) \leq$ $\operatorname{card}\left(\mathcal{P}\left(\tilde{\Lambda}_{\xi}^{\prime}\right)\right) \leq \aleph_{\xi+1}$ 
b) Let $\xi \geq 0$ and set $\xi=\lambda+n$ with $\lambda$ limit and $n<\omega$. For all $u \in W$ we can identify $\tilde{\Phi}_{1+\xi}(u)$ with a couple $\left(\Psi^{\prime}(u), \Psi^{\prime \prime}(u)\right)$ with $\Psi^{\prime}(u)=\bigcup \mathfrak{\bigcup}\left\{\Phi_{1+\eta}\left(\psi_{1+\eta}(u)\right) ;-1 \leq\right.$ $\eta<\lambda\}$ and $\Psi^{\prime \prime}(u)=\bigcup^{\bullet}\left\{\Phi_{\lambda+m}\left(\psi_{\lambda+m}(u)\right) ; 0 \leq m \leq n\right\}$.

Now, identifying the element $\Phi_{1+\eta}\left(\psi_{1+\eta}(u)\right)$ of $\Lambda_{\eta}$ with the singleton

$$
\left\{\Phi_{1+\eta}\left(\psi_{1+\eta}(u)\right)\right\}
$$

we can identify $\Psi^{\prime}(u)$ with a subset of $\Lambda^{\prime}:=\bigcup^{\bullet}\left\{\Lambda_{\eta} ;-1 \leq \eta<\lambda\right\}$, and by the induction hypothesis card $\left.\left(\Lambda^{\prime}\right)\right) \leq \sup \left\{\aleph_{\eta+1} ; \eta<\lambda\right\} \leq \aleph_{\lambda}$. Hence $\Psi^{\prime}(u) \in \mathcal{P}\left(\Lambda^{\prime}\right)$ with $\operatorname{card}\left(\mathcal{P}\left(\Lambda^{\prime}\right)\right) \leq \aleph_{\lambda+1} \leq \aleph_{\xi+1}$.

On the other hand, notice that if $\lambda+m=0$, then $\Phi_{\lambda+m}\left(\psi_{\lambda+m}(u)\right)=0$; and if $\lambda+m \neq 0$, one can always write $\lambda+m=1+\lambda+m^{\prime}$ with $m^{\prime} \leq m$ (in fact $m^{\prime}=m-1$ or $m$, depending whether $\lambda=0$ or not). Hence we always have $\Phi_{\lambda+m}\left(\psi_{\lambda+m}(u)\right) \in \Lambda_{\lambda+m^{\prime}}$ with $0 \leq m^{\prime} \leq m$. It then follows from the induction hypothesis and from part a) above that we can identify $\Psi^{\prime \prime}(u)$ with an element of $\Lambda^{\prime \prime}:=\prod_{m=0}^{n} \Lambda_{\lambda+m^{\prime}}$ with card $\left(\Lambda^{\prime \prime}\right) \leq \aleph_{\lambda+n+1}=\aleph_{\xi+1}$.

This proves that card $\left(\Lambda_{\xi}\right) \leq \aleph_{\xi+1}$ and completes the proof of Lemma 5.9

\section{Proof of the Main Lemma}

In this section we prove Lemma [3.1] which we restate for convenience.

Main Lemma. Assume that $\aleph_{\gamma+1}^{L(\alpha)}<\aleph_{1}$. For any strategy $\sigma \in L(\alpha)$ for Player $I$ in the game $G$, there exists a compact subset $K$ of $2^{\omega}$ satisfying the following conditions:

a) Any $y \in K \backslash Y$ can be constructed by Player $I$ in a run compatible with $\sigma$.

b) Any $z \in Z$ can be constructed by Player II in a non-trivial run, compatible with $\sigma$, and in which Player I constructs some real in $K$.

The proof relies on the results established in the previous section as summarized in Theorem 5.1, which will be our main reference from Section 5. In particular, when we refer to "property $(n)$ " we will mean "property $(n)$ of Theorem 5.1]'.

The first goal is to define the compact set $K$; and for this we need to consider several special types of positions that we shall introduce successively.

6.1. Minimal positions. We shall say that $u$ is a minimal position if:

(1) $u \in V$, and

(2) $\psi_{\gamma}(u)=\min \left\{\psi_{\gamma}\left(u^{\prime}\right) ; \quad u^{\prime} \in V\right.$ and $\left.u^{\prime} \tilde{E} u\right\}$.

When restricted to $V$, the equivalence relation $\tilde{E}$ admits a natural selector inside the set of minimal positions. More precisely:

Lemma 6.2. For any position $v \in V$ there exists a unique position $\mu(v) \in V$ such that $\mu(v)$ is minimal and $\mu(v) \tilde{E} v$. Moreover:

a) If $v^{\prime}$ is a trivial modification of $v$, then $\mu\left(v^{\prime}\right)$ is a trivial modification of $\mu(v)$.

b) If $m \in J_{\gamma}(v)$ and $v_{\uparrow m}$ is minimal, then $\mu(v)_{\uparrow m}=v_{\uparrow m}$.

Proof. Fix $v$ in $V=V_{\gamma}=V_{\gamma+1}$; then by Lemma 5.5 the mapping $\psi_{\gamma}$ is one-to-one on the set $\{w \in V: w \tilde{E} v\}$, so it attains its minimum at a unique point $\mu(v)$ which is clearly the unique minimal position satisfying $\mu(v) \tilde{E} v$. Hence

$$
\psi_{\gamma}(\mu(v))=\min \left\{\psi_{\gamma}(w) ; w \in V, w \tilde{E}_{0} v \text { and } \forall \xi \leq \gamma, \psi_{\xi}(w) E_{\xi} \psi_{\xi}(v)\right\} .
$$


a) Notice that if $v^{\prime} \in V$ is a trivial modification of $v \in V$, then, given any $w \in V$ such that $w \tilde{E}_{0} v$, one can define $w^{\prime} \in V$, a trivial modification of $w$, such that $w^{\prime} \tilde{E}_{0} v^{\prime}$. Applying this observation to $w=\mu(v)$, one gets $w^{\prime} \in V$, a trivial modification of $\mu(v)$, such that $w^{\prime} \tilde{E}_{0} v^{\prime}$; and since $\psi_{\xi}\left(w^{\prime}\right)=\psi_{\xi}(w)=\psi_{\xi}(\mu(v))=$ $\psi_{\xi}(v)$ for all $\xi \leq \gamma$, then $w^{\prime}$ is minimal and $w^{\prime}=\mu\left(v^{\prime}\right)$.

b) If $m \in J_{\gamma}(v)$, then, by Lemma 5.4 $\mu(v)_{\uparrow_{m}} \tilde{E} v_{\uparrow m}$, and it follows from the minimality of $v_{\uparrow_{m}}$ that $\psi_{\gamma}\left(v_{\uparrow_{m}}\right) \leq \psi_{\gamma}\left(\mu(v)_{\uparrow_{m}}\right)$. On the other hand, since $\psi_{\gamma}(\mu(v)) \leq \psi_{\gamma}(v)$, then by the fundamental property of lexicographical ordering we also have $\psi_{\gamma}\left(\mu(v)_{\lceil m}\right) \leq \psi_{\gamma}\left(v_{{ }_{\uparrow m}}\right)$. Hence $\psi_{\gamma}\left(v_{\uparrow_{m}}\right)=\psi_{\gamma}\left(\mu(v)_{\lceil m}\right)$, and again, since $\mu(v)_{{ }_{\uparrow m}} \tilde{E} v_{\uparrow_{m}}$, then by Lemma [5.5] $\mu(v)_{{ }_{\uparrow m}}=v_{\uparrow_{m}}$.

Our next notion will make a crucial use of the set-theoretical assumption " $\aleph_{\gamma+1}^{L(\alpha)}<$ $\aleph_{1}$ " of the Main Lemma. We mention that this is the only place where this assumption is used.

6.3. Good positions. Since $\aleph_{\gamma+1}^{L(\alpha)}<\aleph_{1}$, then by property (2), for all $\xi \leq \gamma$ the equivalence relation $E_{\xi}$ has at most countably many classes, and we can fix a mapping $\mathbf{N}_{\xi}: \Sigma \rightarrow \omega$ satisfying

$$
\left\{\begin{array}{l}
\mathbf{N}_{\xi}(\emptyset)=0 \\
\mathbf{N}_{\xi}(\theta)=\mathbf{N}_{\xi}\left(\theta^{\prime}\right) \Longleftrightarrow \theta E_{\xi} \theta^{\prime} .
\end{array}\right.
$$

Then for any $u \in W$ with $\bar{\chi}_{0}(u)=\left(\xi_{i}\right)_{0 \leq i \leq p}$, we set

$$
\mathbf{N}(u)=\max _{0 \leq i \leq p} \mathbf{N}_{\xi_{i}}\left(\psi_{\xi_{i}}(u)\right) .
$$

We shall say that $u$ is a good position if:

(1) $u$ is a minimal position, and

(2) $\mathbf{N}(u) \leq|u|$.

Lemma 6.4. The set of all good positions of fixed length is finite.

Proof. Let $u$ be a good position and suppose that $n=|u|$ is known; then $\varphi(u)$ which is an element of $(2 \times 2 \times 2)^{n}$ can take only finitely many different values, so we can assume that $\varphi(u)$ is known. Since the characteristic sequence $\tilde{\chi}(u)$ is uniquely determined by $\varphi(u)$, then $\tilde{\chi}_{0}(u)=\left(\xi_{j}\right)_{0<j \leq p}$ is also known. Since $u$ is a good position, the sequence $\tilde{\mathbf{N}}(u)=\left(\mathbf{N}_{\xi_{j}}\left(\psi_{\xi_{j}}(u)\right)_{j \leq p}\right.$ can take only finitely many different values, so again we can assume that $\tilde{\mathbf{N}}(u)$ is known.

Now suppose that $v$ is also a good position with $\varphi(u)=\varphi(v), \tilde{\mathbf{N}}(u)=\tilde{\mathbf{N}}(v)$ and $\tilde{\chi}_{0}(u)=\tilde{\chi}_{0}(v)=\left(\xi_{j}\right)_{0<j \leq p}$. Then for all $0<j \leq p$, since $\mathbf{N}_{\xi_{j}}\left(\psi_{\xi_{j}}(u)\right)=$ $\mathbf{N}_{\xi_{j}}\left(\psi_{\xi_{j}}(v)\right)$, we have $\psi_{\xi_{j}}(u) E_{\xi_{j}} \psi_{\xi_{j}}(v)$. Moreover, for all $\xi \leq \gamma$, if $\xi_{j+1}<\xi \leq \xi_{j}$ (with by convention $\xi_{p+1}=-1$ ), then $\psi_{\xi}(u)=\psi_{\xi_{j}}(u) E_{\xi_{j}} \psi_{\xi_{j}}(v)=\psi_{\xi}(v)$; hence $\psi_{\xi}(u) E_{\xi} \psi_{\xi}(v)$. It follows then from the definitions that $u \tilde{E}_{\gamma} v$, and since $u$ and $v$ are minimal positions, then $\psi_{\gamma}(u)=\psi_{\gamma}(v)$; hence by Lemma 5.5, $u=v$. This shows that $u$ is uniquely determined and finishes the proof of the lemma.

6.5. Admissible positions. We shall say that $v$ is an admissible position if there exists a sequence $\left(u^{(j)}\right)_{j \leq n}$ of good positions satisfying, for all $i, j \leq n$,

(1) $u^{(j)} \tilde{E}_{0} v_{\left.\right|_{j}}$, and

(2) if $i<j$ and $\pi\left(v_{\left.\right|_{i}}\right) R^{\bullet} \pi\left(v_{\left.\right|_{j}}\right)$, then $u^{(i)} \prec u^{(j)}$. 
Such a sequence $\left(u^{(j)}\right)_{j \leq n}$ will be called a sequence of good versions for $v$.

Notice that an admissible position is not necesssarily in $V$; moreover, a sequence of good versions is not unique.

Admissibility is a weak dual form of rule $\left(\mathrm{R}_{2}\right)$ : In fact, if $v$ is admissible, then the mapping $\pi\left(v_{\left.\right|_{k}}\right) \mapsto \psi\left(u^{(k)}\right)$ is a homomorphism for the partial order structures (i.e. an increasing mapping) from the $R^{\bullet}$ ordering to the large extension relation $\preceq$; but this mapping does not (in fact cannot) preserve the height, and so is not a tree homorphism.

Formally speaking, the sets of "good" and "admissible" positions are incomparable. Good positions have a compactness property by Lemma 6.4, and admissible positions will inherit some "goodness" from the sequence of good versions. But the enormous advantage of admissible positions, compared to good ones, is their flexibility, and the following obvious fact is crucial for the definition of $K$ :

Lemma 6.6. The family of all admissible positions is a subtree of $(W, \preceq)$, and hence the set

$$
S=\{\varphi(v): v \in W \text { is an admissible position }\}
$$

is a subtree of $\operatorname{Seq}(2 \times 2 \times 2)$.

Let $K$ denote the projection of $\lceil S\rceil \subset 2^{\omega} \times 2^{\omega} \times 2^{\omega}$ on the first factor.

Lemma 6.7. Any $y \in K \backslash Y$ can be constructed by Player I in a run compatible with $\sigma$.

Proof. Fix $y \in K \backslash Y$. Since $y \in K$, then for all $n$, there exists an admissible position $u_{n}$ such that $\varphi\left(u_{n}\right)=\left(y_{\left.\right|_{n}}, t_{n}, A_{n}\right)$ and $\varphi\left(u_{n}\right) \prec \varphi\left(u_{n+1}\right)$. Fix, for all $n$, a sequence $\left(u_{n}{ }^{(k)}\right)_{k \leq n}$ of good versions for $u_{n}$; since $\varphi\left(u_{n}{ }^{(n)}\right)=\varphi\left(u_{n}\right)$, we can also suppose that $u_{n}=u_{n}{ }^{(n)}$.

Set $\tilde{A}=\left\{\pi\left(u_{n}\right) ; n \in \omega\right\}$, and notice that since card $\left(A_{n}\right)=\left|\pi\left(u_{n}\right)\right|$ for all $n$, the sets $A=\bigcup_{n \in \omega} \uparrow A_{n}$ and $\tilde{A}$ have the same cardinality. It is also clear that $\tilde{A}$ is hereditary for $\prec$. For the rest of the proof we distinguish two cases:

Case 1: $\tilde{A}$ is finite.

In this case $A$ is also finite and $A=A_{m}$ for some $m$, which means that in all the positions $u_{n}$ (with $n \geq m$ ) Player $I I$ 's moves following the $m^{\text {th }}$ move were all trivial. By Lemma 6.4 the set $\left\{u_{n}{ }^{(m)} ; n \geq m\right\}$ is finite; hence there exists an infinite subset $N$ of $\omega \backslash m$ such that the sequence $\left(u_{n}{ }^{(m)}\right)_{n \in N}$ is constant, say with value $v$. Then for all $n \in N$, we have $\pi\left(u_{n}\right)=\pi\left(u_{n}{ }^{(m)}\right)$; hence by admissibility we have $v=u_{n}{ }^{(m)} \prec u_{n}{ }^{(n)}=u_{n}$, and, in particular, $\psi\left(u_{n}\right)=\psi(v)$. Moreover, for any $n^{\prime}>n>m$ we have by assumption $\varphi\left(u_{n}\right) \prec \varphi\left(u_{n^{\prime}}\right)$; hence for $n, n^{\prime} \in N$ we have $u_{n} \prec u_{n^{\prime}}$ and this defines an infinite (trivial) run in which Player $I$ constructs $y$.

Case 2: $\tilde{A}$ is infinite.

In this case $\tilde{A}=\{s: s \prec y\}$, and, since $y \notin Y$, there exists in $\tilde{A}$ by Theorem 2.8 an infinite chain $C$ for $R^{\bullet}$. Fix an enumeration for $C=\left\{\pi\left(u_{k_{j}}\right) ; j \in \omega\right\}$ by some increasing sequence $\left(k_{j}\right)_{j \in \omega}$. The rest of the argument is quite similar to the previous case. By Lemma 6.4, for all $j \in \omega$ the set $\left\{u_{n}{ }^{\left(k_{j}\right)} ; n \geq k_{j}\right\}$ is finite, and hence by a standard diagonal extraction we can find an infinite subset $M$ of $\omega$ such that for all $j \in \omega$, the sequence $\left(u_{n}{ }^{\left(k_{j}\right)}\right)_{n \in M \backslash k_{j}}$ is constant, say with value $v_{j}$.

If $i<j$, pick some $n>k_{j}$ in $N$; then $\pi\left(u_{n}{ }^{\left(k_{i}\right)}\right)=\pi\left(u_{k_{i}}\right) R^{\bullet} \pi\left(u_{k_{j}}\right)=\pi\left(u_{n}{ }^{\left(k_{j}\right)}\right)$. Hence by admissibility $v_{i}=u_{n}{ }^{\left(k_{i}\right)} \prec u_{n}{ }^{\left(k_{j}\right)}=v_{j}$. This proves that the sequence $\left(v_{j}\right)_{j \in \omega}$ defines an infinite run in the game in which Player $I$ constructs $y$. 
Lemma 6.8. Any $z \in Z$ can be constructed by Player II in a non-trivial run, compatible with $\sigma$ and in which Player I constructs some real in $K$.

Proof. Fix $z \in Z$ and choose some $\rho \in \omega_{1}^{\omega}$ such that $(z, \rho) \in\lceil T\rceil$. We shall denote by $W(z)$ the set of all positions $(s, t, \tau)$ such that $t \prec z$, and by $W(z, \rho)$ the set of all positions $v \in W(z)$ in which Player II's last non-trivial move is compatible with $\rho$, that is, of the particular form $\left(i, \rho_{\uparrow_{k}}\right) \in\{0,1\} \times \Omega$ for some $i$ and $k$. Finally, set $V(z)=V \cap W(z)$ and $V(z, \rho)=V \cap W(z, \rho)$.

We shall construct two infinite sequences $\left(u_{n}\right)_{n \in \omega}$ and $\left(v_{n}\right)_{n \in \omega}$ in $V$ such that $u_{0}=v_{0}$ is the unique trivial move in $W(z)$ of length 1 , and, for $n>0$,

(i) $u_{n}$ is a good position,

(ii) $v_{n} \in V(z, \rho)$, and $\left|v_{n}\right|=n+1$,

(iii) $\forall \xi \leq \gamma, \forall m \in J_{\xi}\left(u_{n}\right), u_{n \uparrow m} \tilde{E}_{\xi} u_{m}$,

(iv) $u_{n} \tilde{E} v_{n}$; hence $\varphi\left(u_{n}\right)=\varphi\left(v_{n}\right)$ and $\pi\left(u_{n}\right)=\pi\left(v_{n}\right)=: s_{n}$,

(v) $\varphi\left(v_{n-1}\right) \prec \varphi\left(v_{n}\right)$,

(vi) if $s_{i} R^{+} s_{j}$ and $i<j$, then $v_{i} \prec v_{j}$,

(vii) if $s_{i} R^{\bullet} s_{j}$ and $i<j$, then $u_{i} \prec u_{j}$ and $u_{i} \prec v_{j}$,

(viii) $\lim _{n \rightarrow \infty}\left|s_{n}\right|=\infty$.

We recall that if $\varphi(u)=(s, t, J)$, then $\pi(u) \preceq s$ and $|\pi(u)|=\operatorname{card}(J)$. We first derive the conclusion of the lemma from this construction. So assume that the construction is achieved, and fix some $n$. It follows from (iii) and (iv) that for all $m \leq n, u_{m} \tilde{E}_{0} v_{n \mid m}$, and since the $u_{m}$ 's are good positions, (vi) ensures that $\left(u_{m}\right)_{m \leq n}$ is a sequence of good versions for $v_{n}$; hence $v_{n}$ is an admissible position and $\varphi\left(v_{n}\right) \in S$. Moreover by $(v),\left(\varphi\left(v_{n}\right)\right)_{n \in \omega}$ is an infinite branch of $S$, so it can be identified with a triple $(y, z, a) \in 2^{\omega} \times 2^{\omega} \times 2^{\omega}$, where the first coordinate $y$ is by definition an element of $K$, the second coordinate is precisely the given point $z$, and the third coordinate is the characteristic function of some set $J \subset \omega$ which is infinite by (viii).

It follows from (viii) that $\left\{y_{\left.\right|_{n}}: n \in \omega\right\}=\left\{s_{n}: n \in \omega\right\}$, and by Theorem 2.8 this set contains an infinite chain either for $R^{+}$(in case $y \in Y$ ) or for $R^{\bullet}$ (in case $y \notin Y$ ). Then by conditions (vi) and (vii) we can find an infinite sequence $\left(k_{j}\right)_{j \in \omega}$ in $\omega$ such that either $v_{k_{i}} \prec v_{k_{j}}$ for all $i<j$, or $u_{k_{i}} \prec u_{k_{j}}$ for all $i<j$. In both cases this defines an infinite run $\bar{w}$ in the game with $\varphi(\bar{w})=(y, z, a)$ which satisfies the conclusion.

We shall construct, by induction on $n$, positions $u_{n}$ and $v_{n}$ satisfying conditions (i) to (vii), then, inspecting this construction globally, we will check that condition (viii) also holds.

So let $n>0$ and suppose that the construction is made up to $n-1$. Let $\hat{u}_{n-1}$ and $\hat{v}_{n-1}$ denote the unique trivial extensions (of length $n+1$ ) in $W(z)$ of $u_{n-1}$ and $v_{n-1}$. It is clear that if we set $u_{n}=\hat{u}_{n-1}$ and $v_{n}=\hat{v}_{n-1}$, then conditions (i) to (vii), which are preserved by trivial extensions, are automatically satisfied. However, if we follow this definition forever, then obviously condition (viii) will not be satisfied. So our plan is to define $u_{n}$ and $v_{n}$ differently, namely with a non-trivial last move, unless we meet some obstacle (which we will specify later), in which case we shall define $u_{n}$ and $v_{n}$ to be $\hat{u}_{n-1}$ and $\hat{v}_{n-1}$ respectively.

Let $s^{\prime} \in 2^{n}$ denote the first coordinate of $\varphi\left(u_{n-1}\right)=\varphi\left(v_{n-1}\right)$, that is, the sequence constructed by Player $I$ in both $u_{n-1}$ and $v_{n-1}$. Recall that $\pi\left(u_{n-1}\right)=$ $\pi\left(v_{n-1}\right) \preceq s^{\prime}$, and because $u_{0}$ and $v_{0}$ are already trivial moves, then necessarily 
$\pi\left(u_{n-1}\right)=\pi\left(v_{n-1}\right) \prec s^{\prime}$, and hence $k:=\left|\pi\left(u_{n-1}\right)\right|<n=\left|s^{\prime}\right|$, so that $s:=\left.s^{\prime}\right|_{k+1}$ is already known and we can define

$$
\chi(s)=\left(\xi_{i}, k_{i}\right)_{0 \leq i \leq p} .
$$

Set $J=J_{0}\left(u_{n-1}\right) \cup\{n\}=J_{0}\left(v_{n-1}\right) \cup\{n\}$, and let $\jmath: k+1 \rightarrow J$ denote the increasing enumeration of $J$; finally, set, for all $i \in[0, p]$,

$$
m_{i}=\jmath\left(k_{i}\right) .
$$

Then, by the very definition of the characteristic sequence, for any position $w$ of length $n+1$ such that $\varphi(w) \succ \varphi\left(u_{n-1}\right)$ and in which the last move of Player II is non-trivial, we have

$$
s=\pi(w) \quad \text { and } \quad \tilde{\chi}(w)=\left(\xi_{i}, m_{i}\right)_{0 \leq i \leq p} .
$$

Since $R=R^{\gamma}=R^{\xi_{0}}$, then $s_{m_{0}}=\pi\left(u_{\uparrow_{m_{0}}}\right)=s_{\uparrow_{k_{0}}}$ is the $R$-predecessor of $s$; and since $R$ is generated by $R^{+}$and $R^{\bullet}, s_{m_{0}}$ is either the $R^{+}$-predecessor of $s$, or the $R^{\bullet}$-predecessor of $s$, and we can define

$$
w^{(0)}= \begin{cases}v_{m_{0}} & \text { if } s_{m_{0}} R^{+} s \\ u_{m_{0}} & \text { if } s_{m_{0}} R^{\bullet} s .\end{cases}
$$

Notice that by the induction hypothesis, $w^{(0)} \in V$ and $w^{(0)} \tilde{E} u_{m_{0}}$.

We shall first construct, by (a new) induction, a sequence $\left(w^{(i)}\right)_{0 \leq i \leq p}$ of positions, with $w^{(0)}$ defined as above, and satisfying, for all $i \in[0, p]$,

$$
w^{(i)} \in V_{\xi_{i}}, \quad w^{(i)} \tilde{E}_{\xi_{i}} u_{m_{i}}, \text { and } \quad w^{(i-1)} \prec w^{(i)} \text { if } i>0 .
$$

Suppose that $w^{(i-1)}$ is constructed, and notice that for all $i>0$

$$
\xi_{i}<\xi_{i}+1 \leq \xi_{i-1} .
$$

By condition (i) of the first construction, $u_{m_{i}}$ is in $V$, hence in $V_{\xi_{i}+1}$; and by the induction hypothesis of the second construction $w^{(i-1)}$ is in $V_{\xi_{i-1}}$, hence also in $V_{\xi_{i}+1}$. Also by condition (iii) of the first construction and by the induction hypothesis of the second construction, we have $u_{m_{i}\left\lceil m_{i-1}\right.} \quad \tilde{E}_{\xi_{i-1}} u_{m_{i-1}} \tilde{E}_{\xi_{i-1}} w^{(i-1)}$; hence a fortiori $u_{m_{i}\left\lceil_{m_{i-1}}\right.} \tilde{E}_{\xi_{i}+1} w^{(i-1)}$. Moreover, since $k_{i-1} \in I_{\xi_{i}+1}\left(s_{\uparrow_{k_{i}}}\right.$ ) (see 4.5), then $m_{i-1} \in J_{\xi_{i}+1}\left(u_{m_{i}}\right)$. Then, applying property (6) with $m=m_{i-1}$ and $\eta=\xi_{i}$, we can find $w^{(i)} \in V_{\xi_{i}}$ such that $w^{(i)} \succ w^{(i-1)}$ and $w^{(i)} \tilde{E}_{\xi_{i}} u_{m_{i}}$.

This finishes the construction of the sequence $\left(w^{(i)}\right)_{0 \leq i \leq p}$; and we go back now to the first inductive construction.

Let $h:=h^{+}(s)$ denote the height of $s$ relative to the tree relation $R^{+}$.

We recall that $\left|w^{(p)}\right|=m_{p} \leq n$, and since $w^{(p)} \tilde{E}_{0} u_{m_{p}}$, then $w^{(p)} \in V(z)$. Let $w$ denote the unique extension of $w^{(p)}$ in $W(z)$ of length $n+1$, in which all Player II's moves between $p+1$ and $n-1$ are trivial, and the last move is of the form $\left(z(n), \rho_{\left.\right|_{h}}\right) \in\{0,1\} \times \Omega$.

We first check that $w$ is a legal position in the game. Since $(z, \rho) \in\lceil T\rceil$, then rule $\left(R_{1}\right)$ is satisfied. On the other hand, if $h=1$, then rule $\left(R_{2}\right)$ is automatically satisfied, and if $h>1$, then by Lemma 2.6. $s_{m_{0}}$ is the $R^{+}$-predecessor of $s$; hence $w^{(0)}=v_{m_{0}} \prec w^{(p)} \prec w$; and since by the induction hypothesis $v_{m_{0}} \in W(z, \rho)$, then Player II 's last move in $v_{m_{0}}$ is necessarily $\left(z\left(m_{0}\right), \rho_{\left.\right|_{h-1}}\right)$, and so rule $\left(\mathrm{R}_{2}\right)$ is again satisfied. Moreover, since the last move in $w$ is compatible with $\rho$, then $w \in W(z, \rho)$; but there is no reason for $w$ to be in $V$. 


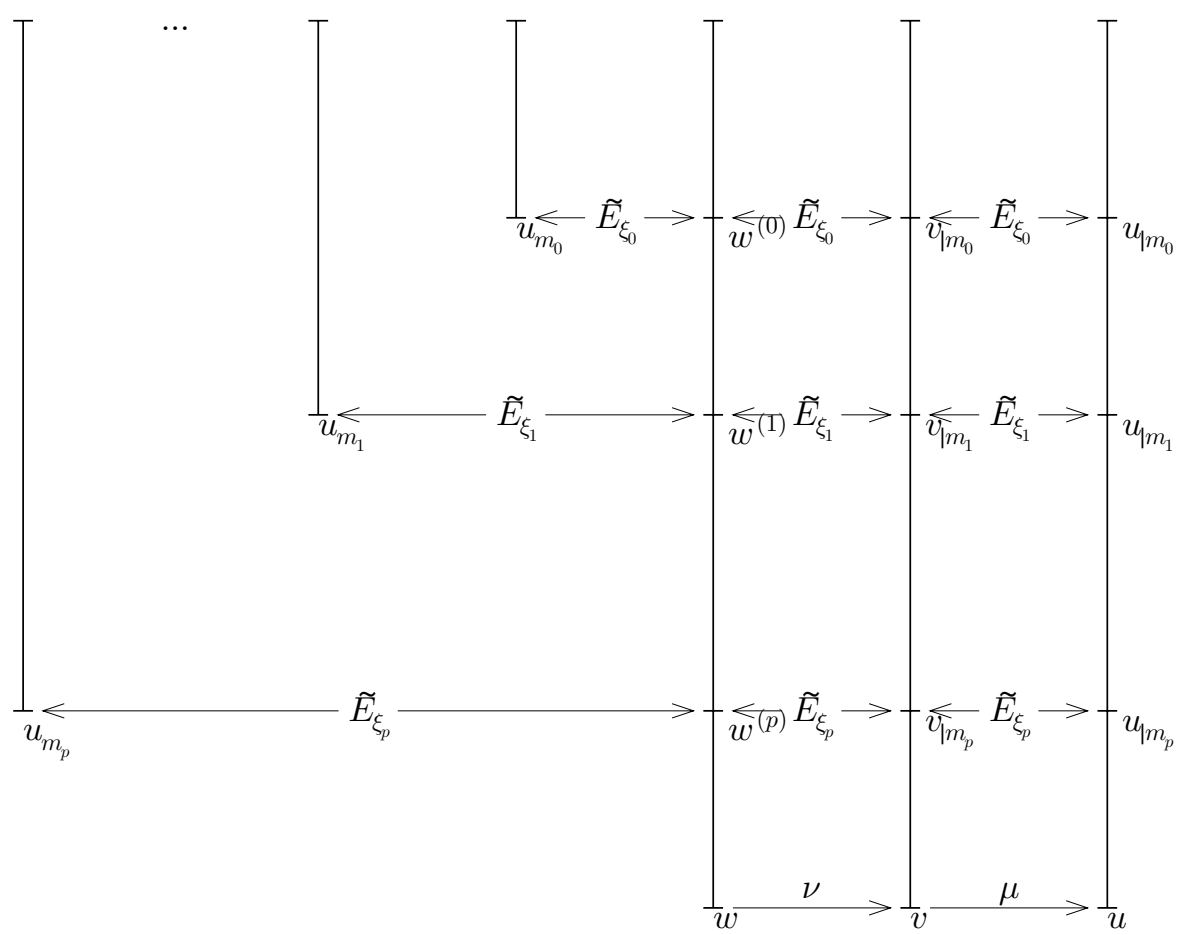

Notice that $\tilde{\chi}(w)=\left(\xi_{i}, m_{i}\right)_{0 \leq i \leq p}$, and for all $i \leq p, w_{\left\lceil m_{i}\right.}=w^{(i)} \in V_{\xi_{i}}$. Hence we can apply property $(7)$ to define $v=\nu(w) \in V$, and then apply Lemma 6.2 to define $u=\mu(v)$. Finally, we set

$$
\left(u_{n}, v_{n}\right)= \begin{cases}(u, v) & \text { if } \mathbf{N}(u) \leq n \\ \left(\hat{u}_{n-1}, \hat{v}_{n-1}\right) & \text { if } \mathbf{N}(u)>n .\end{cases}
$$

As observed before, in the second case conditions (i) to (vii) are automatically fulfilled, so we can assume that $\mathbf{N}(u) \leq n$ and $\left(u_{n}, v_{n}\right)=(u, v)$.

By property (7), $v \in V$ with $|v|=|w|=n+1$ and $\psi_{\gamma}(v)=\psi_{\gamma}(w)$; hence $v \in V(z, \rho)$. By Lemma 6.2, $u=\mu(v)$ is minimal and $u \tilde{E} v$; moreover, since $\mathbf{N}(u) \leq n$, then $u$ is good. This proves (i), (ii) and (iv).

Next, notice that for all $j$

$$
u_{\left\lceil m_{j}\right.} \tilde{E}_{\xi_{j}} v_{\left\lceil m_{j}\right.} \tilde{E}_{\xi_{j}} w_{\left\lceil m_{j}\right.}=w^{(j)} \tilde{E}_{\xi_{j}} u_{m_{j}} .
$$

The first equivalence follows from Lemma 6.2 and property (5), and in a similar way the second equivalence follows from properties (7) and (5); finally, the equality and the last equivalence follow from the construction of $w$. Hence for any $m \in J_{\xi_{j}}\left(u_{n}\right)$ we have $u_{{ }_{\uparrow m}} \tilde{E}_{\xi_{j}} u_{m}$ : For $m=m_{j}$ this follows from the equivalences above, and for $m<m_{j}$ notice that $u_{{ }_{i m}}=\left(u_{\uparrow_{m_{j}}{ }_{\lceil m}}\right.$ and $u_{m}=u_{m_{j} \uparrow_{m}}$, and again apply property (5). Finally, for any $\xi \leq \gamma$ there exists $j \leq p$ such that $\xi_{i+1}<\xi \leq \xi_{i}$ (where by convention $\xi_{p+1}=-1$ ), and then for any $m \in J_{\xi}(u)=J_{\xi_{i}}(u)$, since $\tilde{E}_{\xi_{i}} \subset \tilde{E}_{\xi}$, we have $u_{\uparrow m} \tilde{E}_{\xi} u_{m} ;$ and this proves (iii). 
By construction $u_{m_{p}} \tilde{E}_{0} w^{(p)}$ and $w^{(p)} \preceq w$; and if $w^{*}=w_{\left.\right|_{n}}$, then again by construction all moves in $w^{*} \backslash w^{(p)}$ are trivial. Also, since $\pi\left(u_{n-1}\right)=\pi\left(u_{m_{p}}\right)$, then by condition (vi) of the induction hypothesis $u_{m_{p}} \preceq u_{n-1}$, and by the definition of the characteristic sequence all moves in $u_{n-1} \backslash u_{m_{p}}$ are trivial. Finally, since both $w^{*}$ and $u_{n-1}$ are in $W(z)$, then $\varphi\left(u_{n-1}\right)=\varphi\left(w^{*}\right) \prec \varphi(w)=\varphi(u)$, which proves (v).

For conditions (vi) and (vii), notice first that $s=\pi\left(u_{n}\right)=s_{n}$. Moreover, since $R^{+}$and $R^{\bullet}$ are tree relations, it is enough to check these conditions for $j=n$ and $i$ such that $s_{i}$ is the predecessor of $s_{j}$ for the corresponding relation: $R^{+}$in condition (vi), and $R^{\bullet}$ in condition (vii). We distinguish two cases.

Case 1: $h=1$.

In this case there is nothing to prove for condition (vi) and we now prove (vii).

By Lemma 2.6, $s_{m_{0}}$ is the $R^{\bullet}$-predecessor of $s$; hence $w^{(0)}=u_{m_{0}}$, and by construction $u_{m_{0}}=w^{(0)} \prec w$. Then $w \tilde{E}_{\gamma} v=\nu(w)$ by property (7), and $u_{m_{0}}=w_{\left\lceil m_{0}\right.} \tilde{E}_{\gamma} v_{\left\lceil m_{0}\right.}$ by property (5). Moreover, by property (7) we also have $\psi_{\gamma}(w)=\psi_{\gamma}(v)$, and since $m_{0} \in J_{\gamma}(w)$, it follows then from Lemma 4.4 (ii) that $\psi_{\gamma}\left(u_{m_{0}}\right)=\psi_{\gamma}\left(v_{\left\lceil m_{0}\right.}\right)$; hence, by property (4), $u_{m_{0}}=v_{\left\lceil m_{0}\right.}$, and so $u_{m_{0}} \prec v$. On the other hand, by Lemma 6.2 we also have $u_{\left\lceil m_{0}\right.}=\mu\left(v_{\left\lceil m_{0}\right.}\right)=\mu\left(u_{m_{0}}\right)=u_{m_{0}}$, since $u_{m_{0}}$ is already minimal; hence $u_{m_{0}} \prec u$.

Case 2: $h>1$.

Again by Lemma 2.6, $s_{m_{0}}$ is the $R^{+}$-predecessor of $s$, so $w^{(0)}=v_{m_{0}}$, and by construction $w^{(0)}=v_{m_{0}} \prec w$; moreover, by property $(7), v=\nu(w) \tilde{E} w$. Since $m_{0} \in J_{\gamma}(w)$, then it follows from properties (5) and (7) that $v_{\left\lceil m_{0}\right.} \tilde{E}_{\gamma} v_{m_{0}}$ with $v_{\left\lceil m_{0}\right.}$ and $v_{m_{0}}$ in $V_{\gamma}$, and $\psi_{\gamma}\left(v_{\left\lceil m_{0}\right.}\right)=\psi_{\gamma}\left(v_{m_{0}}\right)$. Hence, by property $(4), v_{\left\lceil m_{0}\right.}=v_{m_{0}}$; that is, $v_{m_{0}} \prec v$, which proves (vi).

Let $s^{\bullet}$ denote the $R^{\bullet}$-predecessor of $s$. Since $s_{m_{0}}$ and $s$ are $R^{+}$-comparable, then, by the definition of a double tree, $s^{\bullet}$ is also the $R^{\bullet}$-predecessor of $s_{m_{0}}$; hence $s^{\bullet}=s_{m}$ for some $m<m_{0}$, and by condition (vii) of the induction hypothesis we have $u_{m} \prec v_{m_{0}} \prec v$, which proves (vii) at level $n$.

This finishes the inductive construction and ensures conditions (i) to (vii).

Fix some $n$ and let $w, v, u$ be the positions constructed at level $n$ as above, and let $w^{\prime}, v^{\prime}, u^{\prime}$ be the corresponding positions constructed at level $n+1$. Assume that $\mathbf{N}(u)>n$, so that $u_{n}$ and $v_{n}$ are defined as trivial extensions of $u_{n-1}$ and $v_{n-1}$, hence necessarily $\pi\left(w^{\prime}\right)=\pi(w)=s$ and $\tilde{\chi}\left(w^{\prime}\right)=\tilde{\chi}(w)=\left(\xi_{i}, m_{i}\right)_{0 \leq i \leq p}$, although $|w|=n+1$ and $\left|w^{\prime}\right|=n+2$. Notice that $w^{*}=w_{\mid n}$ depends only on the sequence $\left(\xi_{i}, m_{i}\right)_{0 \leq i \leq p}$, which was computed before the construction of $w$; hence $w^{*} \prec w^{\prime}$. Moreover, $\psi\left(w^{\prime}\right)=\psi(w)=\psi\left(w^{*}\right) \frown\left\langle\rho_{\left.\right|_{h}}\right\rangle$, where $h=h^{+}(s)$, and so $w^{\prime}$ is a trivial modification (see 4.1) of $w$. Then by property (7), $v^{\prime}$ is a trivial modification of $v$, and by Lemma $6.2 u^{\prime}$ is a trivial modification of $u$; in particular, $\tilde{\chi}\left(u^{\prime}\right)=\tilde{\chi}(u)$ and $\psi\left(u^{\prime}\right)=\psi(u)$. Hence $\mathbf{N}\left(u^{\prime}\right)=\mathbf{N}(u)$, whereas $\left|u^{\prime}\right|=|u|+1$. It follows that after finitely many steps the construction will necessarily reach a level $m>n$ such that $u_{m}$ is not a trivial extension of $u_{m-1}$, hence with $\left|\pi\left(u_{m}\right)\right|>\left|\pi\left(u_{n}\right)\right|$. This proves (viii) and finishes the proof of the lemma.

Lemma 6.7 proves part a) of the Main Lemma, and Lemma 6.8 proves part b). This ends the proof of the Main Lemma, and consequently of the Main Theorem.

Remark 6.9. It is clear that the definition of the game $G$ involves only the tree $R^{+}$, and the proof of part a) of the Main Lemma (that is the proof of Lemma 6.7) uses 
the pair of trees $\left(R^{+}, R^{\bullet}\right)$ (without any additional structure). But the proof of part b) of the Main Lemma requires the complete double tree structure on $\left(R^{+}, R^{\bullet}\right)$, in particular, the interpolation property, which is the main point in the double tree representation. Moreover, the reader can check that this property was used exactly once in the whole proof of the Main Lemma, and this was in the proof of Lemma 6.8, namely inside the construction of the sequence $\left(w^{(i)}\right)_{0 \leq i \leq p}$, where we used the fundamental property $k_{i-1} \in I_{\xi_{i}+1}\left(s_{\uparrow_{k_{i}}}\right)$ for a given characteristic sequence $\chi(s)=\left(\xi_{i}, k_{i}\right)_{0 \leq i \leq p}$, and which, as was pointed out in 4.5 is just a reformulation of the interpolation property. The very long distance separating the introduction of the ad hoc concept of interpolation from its first and only use is probably one of the main difficulties of this proof.

\section{Double trees}

Notation 7.1. In all this section $\mathcal{R}=\left(R^{+}, R^{\bullet}\right)$ denotes a double tree on a countable set $A$. We recall that:

i) $\emptyset$ is the least element for $R^{\bullet}$ and for $R^{+}$.

ii) Any two distinct and non-empty $R^{+}$-comparable elements in $\operatorname{Seq}(A)$ have the same $R^{\bullet}$-predecessor.

iii) $R=R^{+} \circ R^{\bullet}$ is a tree relation on $\operatorname{Seq}(A)$.

For any $s \in \operatorname{Seq}(A)$ we will denote by $s^{\bullet}$ the predecessor of $s$ for $R^{\bullet}$, and by $h^{+}(s)$ and $h^{\bullet}(s)$ the heights of $s$ with respect to the tree relations $R^{+}$and $R^{\bullet}$ (see 2.2). Since $R^{+}$and $R^{\bullet}$ are adapted trees, then $h^{+}(t) \leq|t|$ and $h^{\bullet}(t) \leq|t|$; in particular, $h^{+}(\emptyset)=h^{\bullet}(\emptyset)=0$.

Lemma 7.2. If $s \neq t, s R^{\bullet} t$ and $t R u$, then $s R^{\bullet} u$.

Proof. It is clear if $s=\emptyset$. If $s \neq \emptyset$, since $t R u$, there is a $v$ such that $t R^{+} v$ and $v R^{\bullet} u$. Since $\emptyset \neq s \neq t, s R^{\bullet} t$ and $t R^{+} v$, we have $s R^{\bullet} v$, hence $s R^{\bullet} u$.

Lemma 7.3. If $\emptyset \neq s R$, then $s R^{+} t$ if and only if $h^{\bullet}(s)=h^{\bullet}(t)$.

Proof. If $\emptyset \neq s R^{+} t$, we have

$$
\left\{u: u \neq s \text { and } u R^{\bullet} s\right\}=\left\{u: u \neq t \text { and } u R^{\bullet} t\right\} .
$$

Thus these two sets have the same cardinal, and $h^{\bullet}(s)=h^{\bullet}(t)$.

Conversely, if $s R t$ and $h^{\bullet}(s)=h^{\bullet}(t)$, there is a $u$ such that $s R^{+} u$ and $u R^{\bullet} t$. Then $h^{\bullet}(s)=h^{\bullet}(u)$, and we get $u R^{\bullet} t$ and $h^{\bullet}(u)=h^{\bullet}(t)$; thus $u=t$ and $s R^{+} t$.

Lemma 7.4. If $\emptyset \neq s R t$, then there is a unique $u$ such that $s R^{+} u$ and $u R^{\bullet} t$.

Proof. Assume $s R^{+} u, u R^{\bullet} t, s R^{+} v$ and $v R^{\bullet} t$. Then by Theorem 2.6 we have $u R v$ or $v R u$. Assume without loss of generality that $u R v$. Then there is a $w$ such that $u R^{+} w$ and $w R^{\bullet} v$. Also we get $s R^{+} v, s R^{+} w$ and $w R^{\bullet} t$. Hence $h^{\bullet}(s)=h^{\bullet}(w)=h^{\bullet}(v)$, which implies $w=v$ since $w R^{\bullet} v$.

We then have $u R^{\bullet} t$ and $w R^{\bullet} t$. Since $R^{\bullet}$ is a tree relation, we necessarily have $u R^{\bullet} w$ or $w R^{\bullet} u$, but since $h^{\bullet}(u)=h^{\bullet}(w)$, this implies $u=w=v$.

Lemma 7.5. If $\emptyset \neq s R t R u$ and $s R^{+} u$, then $s R^{+} t R^{+} u$. 
Proof. Since $s R t$, there is a $v$ such that $s R^{+} v$ and $v R^{\bullet} t$; hence $h^{\bullet}(s)=h^{\bullet}(v) \leq$ $h^{\bullet}(t)$. Similarly $h^{\bullet}(t) \leq h^{\bullet}(u)$, but since $s R^{+} u$, we have $h^{\bullet}(s)=h^{\bullet}(u)$. It follows that $h^{\bullet}(s)=h^{\bullet}(t)=h^{\bullet}(u)$, which implies $s R^{+} t$ and $t R^{+} u$, since $s R t$ and $t R u$.

Lemma 7.6. If $s R t R u$ and $s R^{\bullet} u$, then $s R^{\bullet} t$.

Proof. If $s R t$ and $t R u$, there are $v$ and $w$ such that $s R^{+} v, v R^{\bullet} t, t R^{+} w$ and $w R^{\bullet} u$. Then:

- either $v=t$ : then $s R^{+} t, s R^{+} w$ and $w R^{\bullet} u$. But since also $s R^{+} s$ and $s R^{\bullet} u$, Lemma 7.4 implies $s=w$, whence $s=t$ since $s R^{+} t R^{+} w=s$; it follows that $s R^{\bullet} t$

- or $v \neq t$ : then $v R^{\bullet} w R^{\bullet} u$. But since also $s R^{+} v$ and $v R^{\bullet} u$, Lemma 7.4 implies $s=v$; hence $s R^{\bullet} t$.

If $S$ is a tree relation on a set $B$, an infinite branch for $S$ is a sequence $\left(b_{n}\right)_{n \in \omega}$ such that:

i) $b_{n} S b_{n+1}$ and $b_{n} \neq b_{n+1}$ for all $n$, and

ii) for all $b$ such that $b S b_{n}$ for some $n$, there is a $k$ such that $b=b_{k}$.

It is clear that any infinite chain for $S$ is contained in a unique infinite branch. As usual, $S$ is said to be ill-founded if there is some infinite branch for $S$, well-founded if there is no infinite branch for $S$.

Lemma 7.7. $R$ is ill-founded iff $R^{+}$or $R^{\bullet}$ is ill-founded. Moreover, if $R$ has a unique infinite branch, then exactly one of $R^{+}$or $R^{\bullet}$ is ill-founded.

Proof. Since $R^{+} \cup R^{\bullet} \subset R$, it is obvious that if $R^{+}$or $R^{\bullet}$ is ill-founded then $R$ is also ill-founded.

So suppose that $R$ is ill-founded, and let $\left(s_{n}\right)$ be a sequence such that for all $n$ we have $s_{n} \neq s_{n+1}$ and $s_{n} R s_{n+1}$. Then for all $n, h^{\bullet}\left(s_{n}\right) \leq h^{\bullet}\left(s_{n+1}\right)$.

If the non-decreasing sequence $\left(h^{\bullet}\left(s_{n}\right)\right)$ is bounded, there is an integer $n_{0}$ such that $h^{\bullet}\left(s_{n}\right)=h^{\bullet}\left(s_{n+1}\right)$ for all $n \geq n_{0}$. By Lemma 7.3 this implies that $s_{n} R^{+} s_{n+1}$ for all $n \geq n_{0}$, and $\left(s_{n}\right)_{n \geq n_{0}}$ is an infinite chain for $R^{+}$. Conversely, if $\left(s_{n}\right)$ is a branch for $R$ which contains an infinite branch $\left(s_{n_{k}}\right)$ for $R^{+}$, we have $h^{\bullet}\left(s_{n_{k}}\right)=$ $h \bullet\left(s_{n_{0}}\right)$ for all $k$, and the non-decreasing sequence $\left(h \bullet\left(s_{n}\right)\right)$ is bounded.

On the contrary, if the sequence $\left(h^{\bullet}\left(s_{n}\right)\right)$ is unbounded, it contains a subsequence such that $h^{\bullet}\left(s_{n_{k}}\right)<h^{\bullet}\left(s_{n_{k+1}}\right)$. Then there are $\left(t_{k}\right)$ such that $s_{n_{k}} R^{+} t_{k}$ and $t_{k} R^{\bullet} s_{n_{k+1}}$. Since $h^{\bullet}\left(t_{k}\right)=h^{\bullet}\left(s_{n_{k}}\right)<h^{\bullet}\left(s_{n_{k+1}}\right)$, we have $t_{k} \neq s_{n_{k+1}}$, hence $t_{k} R^{\bullet} t_{k+1}$ and $t_{k} \neq t_{k+1}$. It follows that $\left(t_{k}\right)$ generates a branch for $R^{\bullet}$, contained in the branch for $R$ generated by the $s_{n}$ 's. Conversely, if the branch generated by $\left(s_{n}\right)$ contains an infinite branch $\left(t_{k}\right)$ for $R^{\bullet}$, there are integers $n_{k}$ such that $s_{n_{k}} R t_{k}$ and $t_{k} R s_{n_{k+1}}$. Then $h^{\bullet}\left(s_{n_{k}}\right) \leq h^{\bullet}\left(t_{k}\right)<h^{\bullet}\left(t_{k+1}\right) \leq h^{\bullet}\left(s_{n_{k+1}}\right)$, and the sequence $\left(h^{\bullet}\left(s_{n}\right)\right)$ is unbounded.

Finally, it follows from the proof above that if $\left(s_{n}\right)$ is the unique branch of $R$ and if neither $R^{+}$nor $R^{\bullet}$ were well-founded, then the sequence $\left(h^{\bullet}\left(s_{n}\right)\right)$ would be both bounded and unbounded. This contradiction completes the proof. 
Definition 7.8. If $\mathcal{R}$ is a double tree on the set $A$ and $y \in A^{\omega}$, one defines a double tree $\mathcal{R}(y)$ on $A$ by putting

$$
\begin{gathered}
s R(y)^{+} t \Longleftrightarrow s R^{+} t \text { and }(s=t \text { or } s \preceq t \prec y), \\
s R(y)^{\bullet} t \Longleftrightarrow s R^{\bullet} t \text { and }(s=t \text { or } s \preceq t \prec y) .
\end{gathered}
$$

It is then clear that, for every $y \in A^{\omega}$, the relation $R(y):=R^{+}(y) \circ R^{\bullet}(y)$ can also be defined by

$$
s R(y) t \Longleftrightarrow s R t \text { and }(s=t \text { or } s \preceq t \prec y) .
$$

Definition 7.9. A double tree $\mathcal{R}$ on $A$ will be called uniquely branching if for every $y \in A^{\omega}$ there is a unique infinite branch for $R(y)$.

If $\mathcal{R}$ is a uniquely branching double tree on $A$, then the set

$$
\operatorname{ker}(\mathcal{R}):=\left\{y \in A^{\omega}: R^{\bullet}(y) \text { is ill-founded }\right\}=\left\{y \in A^{\omega}: R^{+}(y) \text { is well-founded }\right\}
$$

will be called the kernel of $\mathcal{R}$.

Theorem 7.10. If $\mathcal{R}$ is a uniquely branching double tree on $A$, then the kernel of $\mathcal{R}$ is a Borel subset of $A^{\omega}$.

Proof. Let $P^{\bullet}=\left\{\left(s_{n}\right) \in(\operatorname{Seq}(A))^{\omega}: \forall n s_{n} \neq s_{n+1}\right.$ and $\left.s_{n} R^{\bullet} s_{n+1}\right\}$. Clearly $P^{\bullet}$ is a closed subset of the Polish space $(\operatorname{Seq}(A))^{\omega}$, hence is itself Polish. And if $\bar{s}=\left(s_{n}\right) \in P^{\bullet}$, one has $s_{n} \prec s_{n+1}$ for all $n$ and $\left|s_{n}\right| \rightarrow \infty$. Thus there is a unique $y=\varphi(\bar{s})$ in $A^{\omega}$ such that $s_{n} \prec y$ for all $n$. The function $\varphi$ is continuous from $P^{\bullet}$ onto $\operatorname{ker}(\mathcal{R})$, and this proves that $\operatorname{ker}(\mathcal{R})$ is $\boldsymbol{\Sigma}_{1}^{1}$.

Similarly, let $P^{+}=\left\{\left(s_{n}\right) \in(\operatorname{Seq}(A))^{\omega}: \forall n s_{n} \neq s_{n+1}\right.$ and $\left.s_{n} R^{+} s_{n+1}\right\}$. The same argument as above shows that $A^{\omega} \backslash \operatorname{ker}(\mathcal{R})$ is $\boldsymbol{\Sigma}_{1}^{1}$. Thus $\operatorname{ker}(\mathcal{R})$ is Borel.

Examples 7.11. If $T$ is a tree on $A$ and $\mathcal{R}$ is the double tree defined in Example 2.4, then $\operatorname{ker}(\mathcal{R})=\lceil T\rceil$.

If $Y$ is a $\boldsymbol{\Sigma}_{2}^{0}$ set in $A^{\omega}$ and $\left(T_{i}\right)$ is a sequence of trees on $A$ such that $Y=$ $\bigcup_{i}\left\lceil T_{i}\right\rceil$ and $\operatorname{Seq}(A)=\bigcup_{i} T_{i}$, then the double tree $\mathcal{R}$ defined in Example2.5 satisfies $\operatorname{ker}(\mathcal{R})=Y^{c}$.

\section{Product of double trees}

Since our goal is to associate to each Borel set $Y$ in $A^{\omega}$ a double tree whose kernel is $Y$, we will prove the existence of such double trees by induction on the Borel multiplicative class of $Y$. For this we have to construct some double tree whose kernel is $\bigcap_{n} Y_{n}$ from double trees whose kernels are $A^{\omega} \backslash Y_{n}$. This will be achieved by the product operation that we shall introduce now.

Let $\left(\mathcal{R}_{j}\right)=\left(\left(R_{j}^{+}, R_{j}^{\bullet}\right)\right)_{j}$ be a sequence of double trees on $A$. We define a new double tree $\mathcal{R}=\otimes \mathcal{R}_{j}$ on $A$, which we call the product of the double trees $\left(\mathcal{R}_{j}\right)$. For $s \in \operatorname{Seq}(A)$ we denote by $|s|$ the length of $s$, and by $h_{j}^{+}(s)$ and $h_{j}^{\bullet}(s)$ the heigths of $s$ for the tree relations $R_{j}^{+}$and $R_{j}^{\bullet}$.

Let $E$ be the set of sequences $\left(s_{0}, s_{1}, \ldots, s_{k}\right)$ of elements of $\operatorname{Seq}(A)$ such that

i) $s_{k} \preceq s_{k-1} \preceq \cdots \preceq s_{1} \preceq s_{0}$, and

ii) $\left|s_{j+1}\right|=h_{j}^{+}\left(s_{j}\right)-1$ for $0 \leq j<k$. 
Notice that if $\left(s_{0}, s_{1}, \ldots, s_{k}\right) \in E$, one has

$$
\left|s_{0}\right| \geq h_{0}^{+}\left(s_{0}\right)>\left|s_{1}\right| \geq h_{1}^{+}\left(s_{1}\right)>\ldots>\left|s_{k-1}\right| \geq h_{k-1}^{+}\left(s_{k-1}\right)>\left|s_{k}\right|
$$

thus $\left|s_{0}\right| \geq k$.

Lemma 8.1. For $s \in \operatorname{Seq}(A)$ there is at most one $\left(s_{0}, s_{1}, \ldots, s_{k}\right) \in E$ such that $s_{0} \preceq s$ and

$$
|s|=\left|s_{0}\right| \frac{\left|s_{0}\right|+1}{2}+k+1 .
$$

Proof. It is a well-known fact that the mapping

$$
(n, k) \mapsto \frac{n(n+1)}{2}+k
$$

is one-to-one from $\{(n, k) \in \omega \times \omega: k \leq n\}$ to $\omega$. Hence if $\left(s_{0}, s_{1}, \ldots, s_{k}\right)$ and $\left(t_{0}, t_{1}, \ldots, t_{\ell}\right)$ are in $E$ with $\left|s_{0}\right| \frac{\left|s_{0}\right|+1}{2}+k+1=\left|t_{0}\right| \frac{\left|t_{0}\right|+1}{2}+\ell+1$, we have $k \leq\left|s_{0}\right|$ and $\ell \leq\left|t_{0}\right|$; hence $\left|s_{0}\right|=\left|t_{0}\right|$ and $k=\ell$. Since $s_{0} \preceq s$ and $t_{0} \preceq s$, it follows that $s_{0}=t_{0}$. We now prove by induction on $j \leq k$ that $s_{j}=t_{j}$. If $s_{j}=t_{j}$, then $h_{j}^{+}\left(s_{j}\right)=h_{j}^{+}\left(t_{j}\right)$ and

$$
\left|s_{j+1}\right|=h_{j}^{+}\left(s_{j}\right)-1=h_{j}^{+}\left(t_{j}\right)-1=\left|t_{j+1}\right|,
$$

which proves $s_{j+1}=t_{j+1}$, since $s_{j+1} \preceq s_{0} \preceq s$ and $t_{j+1} \preceq t_{0} \preceq s$.

Notation 8.2. If $s \neq \emptyset$ and $\left(s_{0}, s_{1}, \ldots, s_{k}\right) \in E$ are as in Lemma 8.1 then we set

$$
\begin{aligned}
\rho(s) & =\left(s_{0}, s_{1}, \ldots, s_{k}\right) \\
\rho_{j}(s) & =s_{j} \quad \text { for } 0 \leq j \leq k \\
w(s) & =k .
\end{aligned}
$$

We also set, by convention,

$$
\begin{gathered}
\rho(\emptyset)=\emptyset \\
w(\emptyset)=-1 .
\end{gathered}
$$

Notice that if $\left(s_{0}, s_{1}, \ldots, s_{k}\right) \in E$ and $s_{j}=\emptyset$ for some $j \leq k$, then necessarily $j=k$, since otherwise we would have $\left|s_{j+1}\right|=h_{j}^{+}\left(s_{j}\right)-1=-1$.

We shall denote by $\mathcal{D}(\mathcal{R})$ the domain of the partial mapping $\rho$. We now define on $\operatorname{Seq}(A)$ two relations $R^{+}$and $R^{\bullet}$ as follows:

iii) $s R^{+} t$ iff $s=\emptyset$ or else $s=t$ or else $s$ and $t$ are both in $\mathcal{D}(\mathcal{R})$ and satisfy $s \preceq t, w(s)=w(t), \rho_{j}(s) R_{j}^{+} \rho_{j}(t)$ for $j<k:=w(s)$ and $\rho_{k}(s) R^{\bullet} \rho_{k}(t)$.

iv) $s R^{\bullet} t$ iff $s=t$ or else $s$ and $t$ are both in $\mathcal{D}(\mathcal{R})$ and satisfy $s \preceq t$, $w(s)<w(t), \rho_{j}(s) R^{+} \rho_{j}(t)$ for $j<k:=w(s)$ and $\rho_{k}(s)=\left(\rho_{k}(t)\right)^{\bullet}$.

Notice that in iv) $\left(\rho_{k}(t)\right)^{\bullet}$ is well defined, since $k<w(t)$ implies $\rho_{k}(t) \neq \emptyset$.

Theorem 8.3. $\mathcal{R}=\left(R^{+}, R^{\bullet}\right)$ is a double tree on $A$. Moreover, if $s \in \mathcal{D}(\mathcal{R})$, one has $h^{\bullet}(s)=w(s)+1$.

Proof. By definition $s R^{+} t$ implies $s \preceq t$, and so does $s R^{\bullet} t$. Conditions i) and ii) of 2.3 are obviously satisfied.

We now prove that $R^{+}$is a tree relation. First, if $s, t$ and $u$ are in $\mathcal{D}(\mathcal{R})$ and if $\emptyset \neq s R^{+} t$ and $t R^{+} u$, we have $k=w(s)=w(t)=w(u)$. Moreover, for 
$j<k$, we have $\rho_{j}(s) R_{j}^{+} \rho_{j}(t)$ and $\rho_{j}(t) R_{j}^{+} \rho_{j}(u)$; hence $\rho_{j}(s) R_{j}^{+} \rho_{j}(u)$. Similarly $\rho_{k}(s) R_{k}^{\bullet} \rho_{k}(t)$ and $\rho_{k}(t) R_{k}^{\bullet} \rho_{k}(u)$; hence $\rho_{k}(s) R_{k}^{\bullet} \rho_{k}(u)$. This shows that $s R^{+} u$. Assume now that $s R^{+} u, t R^{+} u$ and $|s| \leq|t|$. We show that $s R^{+} t$. We have $k=w(s)=w(u)=w(t)$. For $j \leq k$ let $s_{j}=\rho_{j}(s), t_{j}=\rho_{j}(t)$. Then

$$
|s|=\left|s_{0}\right| \frac{\left|s_{0}\right|+1}{2}+k+1 \leq\left|t_{0}\right| \frac{\left|t_{0}\right|+1}{2}+k+1=|t|,
$$

hence $\left|s_{0}\right| \leq\left|t_{0}\right|$. We prove by induction on $j<k$ that $s_{j} R^{+} t_{j}$. For $j=0$, since $s_{0} R_{0}^{+} \rho_{0}(u)$ and $t_{0} R_{0}^{+} \rho_{0}(u)$, we have $s_{0} R_{0}^{+} t_{0}$ or $t_{0} R_{0}^{+} s_{0}$. But since $\left|s_{0}\right| \leq\left|t_{0}\right|$, it follows that $s_{0} R_{0}^{+} t_{0}$. Similarly, assuming that $s_{j-1} R_{j-1}^{+} t_{j-1}$, we get $h_{j-1}^{+}\left(s_{j-1}\right) \leq h_{j-1}^{+}\left(t_{j-1}\right)$; hence $\left|s_{j}\right| \leq\left|t_{j}\right|$. And since $s_{j} R_{j}^{+} \rho_{j}(u)$ and $t_{j} R_{j}^{+} \rho_{j}(u)$, this implies that $s_{j} R_{j}^{+} t_{j}$. Finally, for $j=k$, we get $\left|s_{k}\right| \leq\left|t_{k}\right|$, $s_{k} R_{k}^{\bullet} \rho_{k}(u)$ and $t_{k} R_{k}^{\bullet} \rho_{k}(u)$; hence $s_{k} R_{k}^{\bullet} t_{k}$. This completes the proof that $R^{+}$is a tree relation.

We now have to prove that $R^{\bullet}$ is a tree relation. Suppose $s \neq t, t \neq u, s R^{\bullet} t$ and $t R^{\bullet} u$. Then $k=w(s)<w(t)<w(u)$. For $j<k$ we have $\rho_{j}(s) R_{j}^{+} \rho_{j}(t)$ and $\rho_{j}(t) R_{j}^{+} \rho_{j}(u)$; hence $\rho_{j}(s) R_{j}^{+} \rho_{j}(u)$. Moreover, for $j=k$, we have $\rho_{k}(s)=\rho_{k}(t)^{\bullet}$ and $\rho_{k}(t) R_{k}^{+} \rho_{k}(u)$; by Definition $\left[2.3\right.$ iii), this shows that $\rho_{k}(s)=\rho_{k}(u)^{\bullet}$ and $s R^{\bullet} u$.

Assume now that $t \in \mathcal{D}(\mathcal{R})$ and let $k=w(t)$. We show that for $-1 \leq j<k$ there exists a unique $s^{(j)}$ such that $s^{(j)} R^{\bullet} t$ and $w\left(s^{(j)}\right)=j$, and that $s^{(j)} R^{\bullet} s^{(j+1)}$ if $j+1<k$. This will also prove that $h^{\bullet}(t)=w(t)+1$. If $j=-1$, put $s^{(-1)}=\emptyset$.

Now fix $0 \leq j<k$. Then $\rho_{j}(t) \neq \emptyset$, and there exists $s_{j}=\rho_{j}(t)^{\bullet}$ satisfying $\left|s_{j}\right|<\left|\rho_{j}(t)\right|$. By induction on $1 \leq i \leq j$ we now determine the unique $s_{j-i}$ such that $s_{j-i} R_{j-i}^{+} \rho_{j-i}(t)$ and $h_{j-i}^{+}\left(s_{j-i}\right)=1+\left|s_{j-i+1}\right|$, and check that $\left|s_{j-i}\right| \leq$ $\left|\rho_{j-i}(t)\right|$. If $s_{j+1-i}$ is determined, satisfying $s_{j+1-i} R_{j+1-i}^{+} \rho_{j+1-i}(t)$, one has $\left|s_{j+1-i}\right| \leq\left|\rho_{j+1-i}(t)\right|=h_{j-i}^{+}\left(\rho_{j-i}(t)\right)-1$. Thus there exists a unique $s_{j-i}$ such that $s_{j-i} R_{j-i}^{+} \rho_{j-i}(t)$ and $h_{j-i}^{+}\left(s_{j-i}\right)=1+\left|s_{j+1-i}\right|$. Then $\left(s_{0}, s_{1}, \ldots, s_{j}\right) \in E$. Also, if $\ell=\left|s_{0}\right| \frac{\left|s_{0}\right|+1}{2}+j+1$, it is easily checked that $s^{(j)}=t_{\mid \ell}$ satisfies $w\left(s^{(j)}\right)=j$ and $s^{(j)} R^{\bullet} t$, and that $s^{(j)}$ is the only one which satisfies these properties.

If $s^{\prime}$ is constructed with $w\left(s^{\prime}\right)=j$ from $s^{(j+1)}$ in the same way as we constructed $s^{(j)}$ from $t$, we have $s^{\prime} R^{\bullet} s^{(j+1)}$ and $s^{(j+1)} R^{\bullet} t$; hence $s^{\prime} R^{\bullet} t$ and $w\left(s^{\prime}\right)=j$. By uniqueness this implies that $s^{\prime}=s^{(j)}$; hence that $s^{(j)} R^{\bullet} s^{(j+1)}$, and completes the proof that $R^{\bullet}$ is a tree relation.

Finally, if $t R^{+} u, s \neq u$ and $s \neq t$, notice that $k=w(t)=w(u)$, and that $s R^{\bullet} u$ iff $j=w(s)<k, \rho_{j}(s)=\rho_{j}(u)^{\bullet}$ and $\rho_{i}(s) R^{+} \rho_{i}(u)$ for $i<j$. Also $s R^{\bullet} t$ iff $j=w(s)<k, \rho_{j}(s)=\rho_{j}(t)^{\bullet}$ and $\rho_{i}(s) R^{+} \rho_{i}(t)$ for $i<j$. Since $j<w(t)=w(u)$ and $t R^{+} u$, we have $\rho_{j}(t) R_{j}^{+} \rho_{j}(u)$; hence $\rho_{j}(t)^{\bullet}=\rho_{j}(u)^{\bullet}$. This shows that $s R^{\bullet} t \Longleftrightarrow s R^{\bullet} u$, and completes the proof of the theorem.

Lemma 8.4. Assume $\emptyset \neq s \neq t, \rho(s)=\left(s_{0}, s_{1}, \ldots, s_{k}\right)$ and $\rho(t)=\left(t_{0}, t_{1}, \ldots, t_{\ell}\right)$. Then $s R t$ iff $k=w(s) \leq w(t), s_{j} R_{j}^{+} t_{j}$ for $j<k, s_{k} \neq t_{k}$ and $s_{k} R_{k}^{\bullet} t_{k}$.

In particular, if $s R t$, then $s_{j} R_{j} t_{j}$ for all $j \leq w(s)$.

Proof. If $s R t$, there is a $u$ such that $s R^{+} u$ and $u R^{\bullet} t$. Then $w(s)=w(u) \leq w(t)$. Moreover, for $j<k=w(s)$, we have $s_{j} R_{j}^{+} \rho_{j}(u)$ and $\rho_{j}(u) R_{j}^{+} t_{j}$; hence $s_{j} R_{j}^{+} t_{j}$. 
Finally, $s_{k} R_{k}^{\bullet} \rho_{k}(u)$ and either $t=u \neq s$, hence $t_{k}=\rho_{k}(u) \neq s_{k}$, or $\rho_{k}(u)=t_{k}^{\bullet}$. Thus $s_{k} R_{k}^{\bullet} t_{k}$ and $s_{k} \neq t_{k}$.

Conversely, if the conditions of the lemma are satisfied, either $k=w(s)=w(t)$ and $s R^{+} t$, or $w(s)<w(t)$ and $s_{k} R_{k}^{\bullet} t_{k}^{\bullet}$. In the second case, it is possible to construct by downward induction as in the previous theorem some $\left(u_{0}, u_{1}, \ldots, u_{k}\right) \in$ $E$ such that $u_{k}=t_{k}^{\bullet}$ and $u_{j} R_{j}^{+} t_{j}$ for $j<k$. Also, there is a $u \preceq t$ such that $w(u)=k$ and $\rho(u)=\left(u_{0}, u_{1}, \ldots, u_{k}\right)$; then we have $s R^{+} u$ and $u R^{\bullet} t$, whence $s R t$.

Lemma 8.5. If $\mathcal{R}=\bigotimes \mathcal{R}_{j}$, then for every y in $A^{\omega}$ one has $\mathcal{R}(y)=\bigotimes \mathcal{R}_{j}(y)$.

This follows immediately from the definitions.

Theorem 8.6. If each $\mathcal{R}_{j}$ is a uniquely branching double tree on $A$, then so is $\otimes \mathcal{R}_{j}$.

Proof. We assume first that each $\mathcal{R}_{j}(y)$ has an infinite branch for $R_{j}(y)$, and prove that $\mathcal{R}(y)$ has an infinite branch for $R(y)$.

If $\mathcal{R}_{i}(y)$ has an infinite branch for $R_{i}^{\bullet}(y)$ for some $i$, there is a $k$ such that $\mathcal{R}_{k}(y)$ has an infinite branch $\left(s_{k, p}\right)_{p}$ for $R_{k}^{\bullet}(y)$ and $\mathcal{R}_{i}(y)$ has no infinite branch for $R_{i}^{\bullet}(y)$ if $i<k$. But then for $i<k, \mathcal{R}_{i}(y)$ has an infinite branch for $R_{i}^{+}(y)$, and we can find for $i<k$ and $j \in \omega$ elements $s_{i, j} \preceq y$ such that $h_{i}^{+}\left(s_{i, j}\right)=j$ and $s_{i, j} R_{i}^{+} s_{i, j+1}$. And if we define by downward induction integers $j_{i, p}$ for $i<k$ and $p \in \omega$ such that $j_{i-1, p}=1+\left|s_{i, j_{i, p}}\right|$, it is easy to check that $\left(s_{0, j_{0, p}}, s_{1, j_{1, p}}, \ldots, s_{k-1, j_{k-1}, p}, s_{k, p}\right)$ belongs to $E$ for all $p$. Moreover if

$$
\ell_{p}:=\left|s_{0, j_{0, p}}\right| \frac{\left|s_{0, j_{0, p}}\right|+1}{2}+k+1,
$$

then $s^{(p)}:=y_{\mid \ell_{p}}$ satisfies $w\left(s^{(p)}\right)=k, \rho\left(s^{(p)}\right)=\left(s_{0, j_{0, p}}, s_{1, j_{1, p}}, \ldots, s_{k-1, j_{k-1, p}}, s_{k, p}\right)$, and $s^{(p)} R^{+} s^{(p+1)}$ for all $p$; so we conclude that $\otimes \mathcal{R}_{j}(y)$ has an infinite branch for $R^{+}(y)$, hence for $R(y)$.

On the contrary, if each $\mathcal{R}_{j}(y)$ has an infinite branch for $R_{j}^{+}(y)$, we now prove that $\mathcal{R}(y)$ has an infinite branch for $R^{\bullet}(y)$.

As above, we can find for every $i$ and $j \in \omega$ elements $s_{i, j} \preceq y$ such that $h_{i}^{+}\left(s_{i, j}\right)=$ $j$ and $s_{i, j} R_{i}^{+} s_{i, j+1}$. Denote $t_{i}=s_{i, 0}^{\bullet}$. By $2.3 \mathrm{iii}$ ), we see that $s_{i, j}^{\bullet}=s_{i, 0}^{\bullet}$ for all $j$. And if we define by downward induction integers $j_{i, p}$ for $i<p$ and $p \in \omega$ such that $j_{p-1, p}=1+\left|t_{p}\right|$ and $j_{i-1, p}=1+\left|s_{i, j_{i, p}}\right|$ for $i<p$, it is easy to check that $\left(s_{0, j_{0, p}}, s_{1, j_{1, p}}, \ldots, s_{p-1, j_{p-1, p}}, t_{p}\right)$ belongs to $E$ for all $p$. Moreover, if

$$
\ell_{p}:=\left|s_{0, j_{0, p}}\right| \frac{\left|s_{0, j_{0, p}}\right|+1}{2}+p+1,
$$

then $s^{(p)}:=y_{\mid \ell_{p}}$ satisfies $w\left(s^{(p)}\right)=p, \rho\left(s^{(p)}\right)=\left(s_{0, j_{0, p}}, s_{1, j_{1, p}}, \ldots, s_{p-1, j_{p-1, p}}, t_{p}\right)$, and $s^{(p)} R^{\bullet} s^{(p+1)}$ for all $p$; so we conclude that $\otimes \mathcal{R}_{j}(y)$ has an infinite branch for $R^{\bullet}(y)$, hence for $R(y)$.

Conversely, if $\otimes \mathcal{R}_{j}(y)$ has an infinite branch $\left(s^{(p)}\right)$ for $R^{+}(y)$, we have $w\left(s^{(p)}\right)=$ $k=w\left(s^{(0)}\right)$ for each $p$. Then we have $\rho_{j}\left(s^{(p)}\right) R_{j}^{+} \rho_{j}\left(s^{(p+1)}\right)$ for all $p$ if $j<k$, and $\rho_{k}\left(s^{(p)}\right) R_{k}^{\bullet} \rho_{k}\left(s^{(p+1)}\right)$ for all $p$. This means that $\left(\rho_{j}\left(s^{(p)}\right)\right)_{p}$ is an infinite branch for $R_{j}^{+}(y)$ if $j<k$, and that $\left(\rho_{k}\left(s^{(p)}\right)\right)_{p}$ is an infinite branch for $R_{k}^{\bullet}(y)$. 
Similarly, if $\otimes \mathcal{R}_{j}(y)$ has an infinite branch $\left(s^{(p)}\right)$ for $R^{\bullet}(y)$ with $h^{\bullet}\left(s^{(p)}\right)=$ $p+1$, it is easily checked that $\rho_{j}\left(s^{(p)}\right) R_{j}^{+} \rho_{j}\left(s^{(p+1)}\right)$ for $j<p$. This means that $\left(\rho_{j}\left(s^{(p)}\right)\right)_{p>j}$ is an infinite branch for $R_{j}^{+}(y)$ for all $j$.

In either case we check that the only possible infinite branch for $R(y)$ is the one constructed in the first part of the proof. It follows that, for every $y \in A^{\omega}$, there is a unique infinite branch in $\operatorname{Seq}(A)$ for $R(y)$.

Theorem 8.7. If $\left(\mathcal{R}_{j}\right)$ is a sequence of uniquely branching double trees on $A$, then

$$
\operatorname{ker}\left(\bigotimes \mathcal{R}_{j}\right)=A^{\omega} \backslash \bigcup_{p} \operatorname{ker}\left(\mathcal{R}_{p}\right)
$$

Proof. Let $K_{j}:=\operatorname{ker}\left(\mathcal{R}_{j}\right)$. If $y \notin \bigcup_{j} K_{j}$, then there is in each $\mathcal{R}_{j}$ an infinite branch for $R_{j}^{+}(y)$, and it follows immediately from the proof of the above theorem that there is then an infinite branch for $R^{\bullet}(y)$ in $\otimes \mathcal{R}_{j}(y)$.

And if $y \in \bigcup K_{j}$, there is a $p$ such that $y \notin K_{j}$ for $j<p$ and $y \in K_{p}$. There is an infinite branch in $\mathcal{R}_{j}(y)$ for $R_{j}^{+}(y)$ if $j<p$, and an infinite branch in $\mathcal{R}_{p}(y)$ for $R_{p}^{\bullet}(y)$. It follows immediately from the proof of the above theorem that there is then an infinite branch for $R^{+}(y)$ in $\otimes \mathcal{R}_{j}(y)$.

Thus $y \in \operatorname{ker}\left(\otimes \mathcal{R}_{j}\right) \Longleftrightarrow \forall p \quad y \notin \operatorname{ker}\left(\mathcal{R}_{p}\right)$.

\section{Interpolation of DOUble trees}

In this section we introduce the derivatives of a double tree $\mathcal{R}=\left(R^{+}, R^{\bullet}\right)$ in order to define the interpolation family $\left(R^{\xi}\right)_{\xi \leq \gamma}$ we need. The main theorem of this section will state that the product of a sequence of interpolable double trees is interpolable too.

Definition 9.1. If $\mathcal{R}$ is a double tree on $A$ and $S$ is a relation on $\operatorname{Seq}(A)$, the derivative $D(S)$ is

$$
D(S)=\bigcup_{m \in \omega} D_{m}(S)
$$

where the sets $D_{m}(S)$ are inductively defined by

$$
\begin{aligned}
D_{0}(S) & \left.=\{s, t) \in \operatorname{Seq}(A)^{2}: s=t\right\}, \\
D_{m+1}(S) & =\left\{(s, t) \in \operatorname{Seq}(A)^{2}: \exists u, u^{\prime} \quad u \preceq t, u R u^{\prime},(s, u) \in D_{m}(S), t S u^{\prime}\right\} .
\end{aligned}
$$

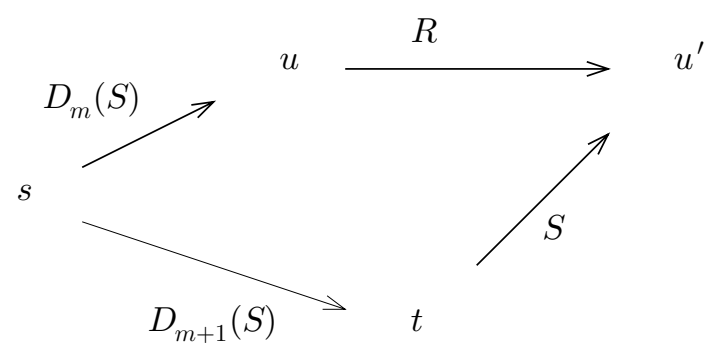

Lemma 9.2. If $S$ is a tree relation finer than the extension relation and if $R$ is finer than $S$, then $R \subset D(S) \subset S$. 
Proof. We prove first that $R \subset D_{1}(S)$. If $s R t$, take $u=s$ and $u^{\prime}=t$. Then $u \preceq t$, $(s, u) \in D_{0}(S)$ and $t S u^{\prime}$, which proves $R \subset D_{1}(S) \subset D(S)$.

We now have to prove that $D_{m}(S) \subset S$ for all integers $m$. This is shown by induction on $m$. Since $S$ is reflexive, we have $D_{0}(S) \subset S$.

And if $D_{m}(S) \subset S$ and $s, t, u, u^{\prime}$ satisfy $u \preceq t, u R u^{\prime},(s, u) \in D_{m}(S) \subset S$, $t S u^{\prime}$, one has $u S u^{\prime}$ since $R \subset S$; hence $s S u^{\prime}$. Since $S$ is a tree relation, it follows from $s S u^{\prime}$ and $t S u^{\prime}$ that $s S t$ or $t S s$. But since $s \preceq u \preceq t$, this implies that $s S t$. Thus $D_{m+1}(S) \subset S$. So $D(S)=\bigcup_{m} D_{m}(S) \subset S$.

Definition 9.3. If $\mathcal{R}=\left(R^{+}, R^{\bullet}\right)$ is a double tree on $A$, we define inductively

$$
\begin{aligned}
D^{0}(\mathcal{R}) & =\left\{(s, t) \in \operatorname{Seq}(A)^{2}: s \preceq t\right\}, \\
D^{\xi+1}(\mathcal{R}) & =D\left(D^{\xi}(\mathcal{R})\right), \\
D^{\lambda}(\mathcal{R}) & =\bigcap_{\xi<\lambda} D^{\xi}(\mathcal{R}) \quad \text { for limit } \lambda .
\end{aligned}
$$

Remark 9.4. If $\mathcal{R}=\left(R^{+}, R^{\bullet}\right)$ is in $L(\alpha)$ for some $\alpha \in \omega^{\omega}$, then the family $\left(D^{\xi}(\mathcal{R})\right)_{\xi<\omega_{1}}$ is also in $L(\alpha)$.

This follows straightforwardly from the formulas above which define the derivatives of $\mathcal{R}$.

Theorem 9.5. Let $\mathcal{R}$ be a double tree on $A$. Then:

i) For all $\xi, D^{\xi}(\mathcal{R})$ is a tree relation on $\operatorname{Seq}(A)$.

ii) For $\eta \leq \xi$, one has $R \subset D^{\xi}(\mathcal{R}) \subset D^{\eta}(\mathcal{R})$.

iii) If $s \preceq t,(s, u) \in D^{\xi+1}(\mathcal{R})$ and $(t, u) \in D^{\xi}(\mathcal{R})$, then $(s, t) \in D^{\xi+1}(\mathcal{R})$.

Proof. This will be proved by induction on $\xi$. It is clear that $D^{0}(\mathcal{R})$ is a tree relation and that $R \subset D^{0}(\mathcal{R})$. Hence i) and ii) hold for $\xi=0$.

Assume now that for $\eta<\xi$ we have:

(1) If $s, t, u$ satisfy $(s, t) \in D^{\eta}(\mathcal{R})$ and $(t, u) \in D^{\eta}(\mathcal{R})$, then $(s, u) \in D^{\eta}(\mathcal{R})$.

(2) If $s, t, u$ satisfy $s \preceq t$ and $(s, u) \in D^{\eta}(\mathcal{R})$ and $(t, u) \in D^{\eta}(\mathcal{R}), \quad$ then $(s, t) \in$ $D^{\eta}(\mathcal{R})$.

(3) For $\zeta \leq \eta, \quad R \subset D^{\eta}(\mathcal{R}) \subset D^{\zeta}(\mathcal{R})$.

We have to prove the following three assertions:

(4) For $\eta<\xi, \quad R \subset D^{\xi}(\mathcal{R}) \subset D^{\eta}(\mathcal{R})$.

(5) If $s, t, u$ satisfy $(s, t) \in D^{\xi}(\mathcal{R})$ and $(t, u) \in D^{\xi}(\mathcal{R}), \quad$ then $(s, u) \in D^{\xi}(\mathcal{R})$.

(6) If $s, t, u$ satisfy $s \preceq t,(s, u) \in D^{\xi}(\mathcal{R})$ and $(t, u) \in D^{\xi}(\mathcal{R}), \quad$ then $(s, t) \in$ $D^{\xi}(\mathcal{R})$.

Notice first that conditions (5) and (6) imply that $D^{\xi}(\mathcal{R})$ is a tree relation: $D^{\xi}(\mathcal{R})$ is transitive by $(5)$, and if $s, t, u$ satisfy $(s, u) \in D^{\xi}(\mathcal{R})$ and $(t, u) \in D^{\xi}(\mathcal{R})$, we have to prove that $(s, t) \in D^{\xi}(\mathcal{R})$ or $(t, s) \in D^{\xi}(\mathcal{R})$. But since $s \preceq u$ and $t \preceq u$, either $s \preceq t$ or $t \preceq s$. Then the conclusion follows from (6).

If $\xi$ is a limit ordinal, we have $D^{\xi}(\mathcal{R})=\bigcap_{\zeta<\xi} D^{\zeta}(\mathcal{R})$; hence, by (3), for every $\eta<\xi$

$$
R \subset \bigcap_{\zeta<\xi} D^{\zeta}(\mathcal{R})=D^{\xi}(\mathcal{R}) \subset D^{\eta}(\mathcal{R})
$$

which proves (4). If $(s, t) \in D^{\xi}(\mathcal{R})$ and $(t, u) \in D^{\xi}(\mathcal{R})$, we have, for $\eta<\xi$, $(s, t) \in D^{\eta}(\mathcal{R})$ and $(t, u) \in D^{\eta}(\mathcal{R})$; thus $(s, u) \in D^{\eta}(\mathcal{R})$ by (1). And since this holds for all $\eta<\xi$, we get $(s, u) \in D^{\xi}(\mathcal{R})$, hence (5). 


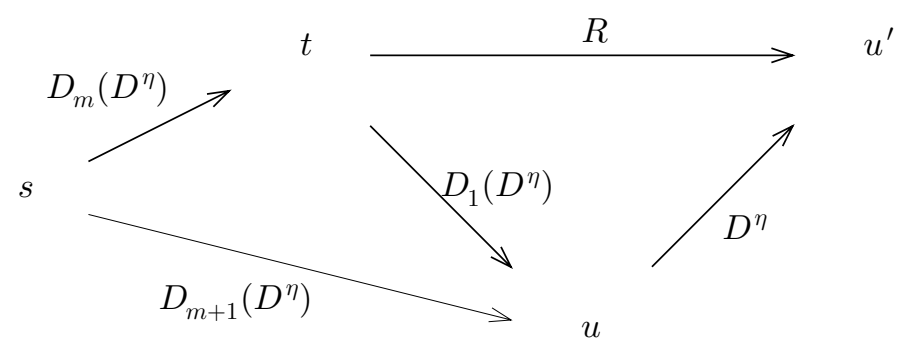

FiguRE 1.

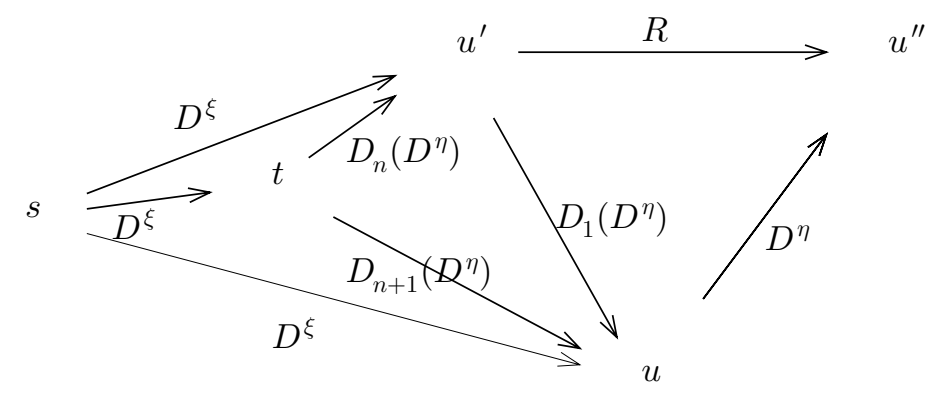

FIGURE 2.

If $s \preceq t,(s, u) \in D^{\xi}(\mathcal{R})$ and $(t, u) \in D^{\xi}(\mathcal{R})$, we have, for $\eta<\xi,(s, u) \in D^{\eta}(\mathcal{R})$ and $(t, u) \in D^{\eta}(\mathcal{R})$; thus $(s, t) \in D^{\eta}(\mathcal{R})$ by $(2)$. And since this holds for all $\eta<\xi$, we get $(s, t) \in D^{\xi}(\mathcal{R})$, hence $(6)$.

If $\xi=\eta+1, D^{\eta}(\mathcal{R})$ is a tree relation from (1) and (2). It follows then from Lemma 9.2 that $R \subset D^{\xi}(\mathcal{R})=D^{\eta+1}(\mathcal{R})=D\left(D^{\eta}(\mathcal{R})\right) \subset D^{\eta}(\mathcal{R})$. Then, if $\zeta<\xi$, we have $\zeta \leq \eta$; hence by $(1), R \subset D^{\xi}(\mathcal{R}) \subset D^{\eta}(\mathcal{R}) \subset D^{\zeta}(\mathcal{R})$. Thus (4) is proved.

Assume now that $(s, t) \in D_{m}\left(D^{\eta}(\mathcal{R})\right)$ and $(t, u) \in D_{1}\left(D^{\eta}(\mathcal{R})\right)$. By definition $t \preceq u$, and there exists $u^{\prime}$ such that $t R u^{\prime}$ and $\left(u, u^{\prime}\right) \in D^{\eta}(\mathcal{R})$ (see Figure 1). This proves that $(s, u) \in D_{m+1}\left(D^{\eta}(\mathcal{R})\right)$. So it is shown by induction on $m$ that if $(s, t) \in \mathcal{D}^{\xi}(\mathcal{R})=\bigcup_{m} D_{m}\left(D^{\eta}(\mathcal{R})\right)$ and $(t, u) \in D_{1}\left(D^{\eta}(\mathcal{R})\right)$, then $(s, u) \in \mathcal{D}^{\xi}(\mathcal{R})$.

The following step is to prove by induction on $n$ that if $(s, t) \in \mathcal{D}^{\xi}(\mathcal{R})$ and $(t, u) \in$ $D_{n}\left(D^{\eta}(\mathcal{R})\right)$, then $(s, u) \in \mathcal{D}^{\xi}(\mathcal{R})$. The statement is obvious for $n=0$, and we just proved it for $n=1$. Suppose that $(s, t) \in \mathcal{D}^{\xi}(\mathcal{R})$ and $(t, u) \in D_{n+1}\left(D^{\eta}(\mathcal{R})\right)$; by definition, there are $u^{\prime}$ and $u^{\prime \prime}$ such that $\left(t, u^{\prime}\right) \in D_{n}\left(D^{\eta}(\mathcal{R})\right), u^{\prime} \preceq u, u^{\prime} R u^{\prime \prime}$ and $\left(u, u^{\prime \prime}\right) \in D^{\eta}(\mathcal{R})$ (see Figure 2). Then $\left(u^{\prime}, u\right) \in D_{1}\left(D^{\eta}(\mathcal{R})\right.$ ), and by the inductive hypothesis $\left(s, u^{\prime}\right) \in D^{\xi}(\mathcal{R})$. By the above argument, this shows that $(s, u) \in D^{\xi}(\mathcal{R})$, and completes the proof of $(5)$, since $D^{\xi}(\mathcal{R})=\bigcup_{m} D_{m}\left(D^{\eta}(\mathcal{R})\right)$. 


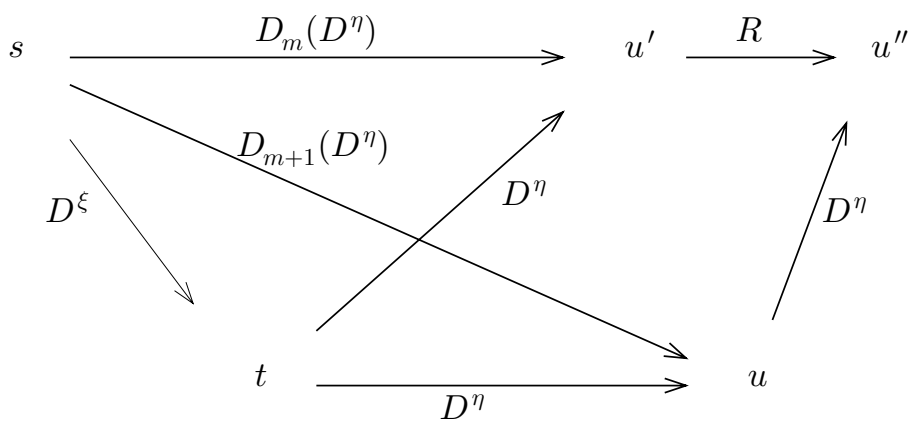

FIGURE 3.

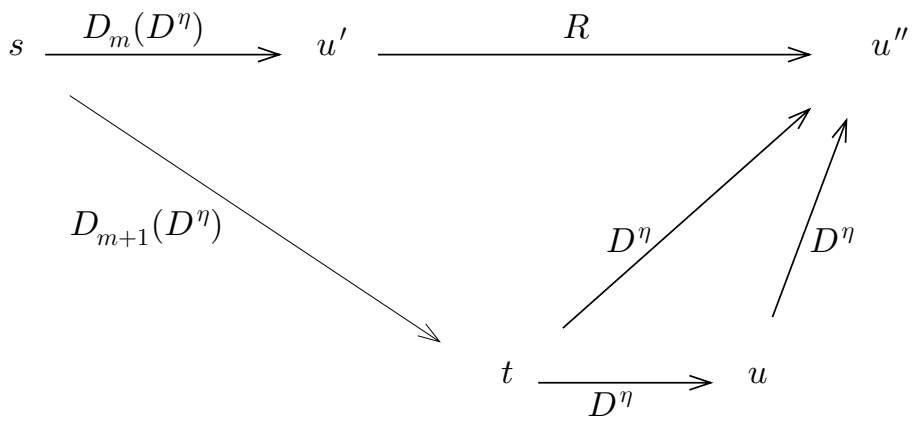

FiguRE 4.

Finally, since $D^{\xi}(\mathcal{R}) \subset D^{\eta}(\mathcal{R}),(6)$ will follow from

if $s, t, u$ satisfy $s \preceq t$ and $(s, u) \in D^{\xi}(\mathcal{R})$ and $(t, u) \in D^{\eta}(\mathcal{R})$,

$$
\text { then }(s, t) \in D^{\xi}(\mathcal{R}) \text {, }
$$

which will also prove 9.5 iii) for $\eta$. Since $D^{\xi}(\mathcal{R})=\bigcup_{m} D_{m}\left(D^{\eta}(\mathcal{R})\right)$, this will be done by proving by induction on $m$ that

$$
\text { if } s, t, u \text { satisfy } s \preceq t,(s, u) \in D_{m}\left(D^{\eta}(\mathcal{R})\right) \text { and }(t, u) \in D^{\eta}(\mathcal{R}),
$$
then $(s, t) \in D^{\xi}(\mathcal{R})$.

If $m=0$, we have $s \preceq t \preceq u=s$; hence $s=t$, and the conclusion is obvious. Assume then (8) holds for $m$ and let $s, t, u$ satisfy $s \preceq t,(s, u) \in D_{m+1}\left(D^{\eta}(\mathcal{R})\right)$, and $(t, u) \in D^{\eta}(\mathcal{R})$. By the definition of $D_{m+1}$ there are $u^{\prime}$ and $u^{\prime \prime}$ satisfying $u^{\prime} \preceq u \preceq u^{\prime \prime}, u^{\prime} R u^{\prime \prime}$ and $\left(u, u^{\prime \prime}\right) \in D^{\eta}(\mathcal{R})$. Since $D^{\eta}(\mathcal{R})$ is a tree relation by $(5)$ and (6), we have $\left(t, u^{\prime \prime}\right) \in D^{\eta}(\mathcal{R})$. We also have $\left(u^{\prime}, u^{\prime \prime}\right) \in D^{\eta}(\mathcal{R})$ by $(4)$; hence either $\left(t, u^{\prime}\right) \in D^{\eta}(\mathcal{R})$ or $\left(u^{\prime}, t\right) \in D^{\eta}(\mathcal{R})$.

In the first case (see Figure 3), we have $s \preceq t,\left(s, u^{\prime}\right) \in D_{m}\left(\mathcal{D}^{\eta}(\mathcal{R})\right)$ and $\left(t, u^{\prime}\right) \in$ $D^{\eta}(\mathcal{R})$; hence $(s, t) \in D^{\xi}(\mathcal{R})$, since $(8)$ holds for $m$. 
In the second case (see Figure 4), we have $u^{\prime} \preceq t \preceq u^{\prime \prime}, u^{\prime} R u^{\prime \prime},\left(t, u^{\prime \prime}\right) \in D^{\eta}(\mathcal{R})$, and $\left(s, u^{\prime}\right) \in D_{m}\left(D^{\eta}(\mathcal{R})\right)$; hence $(s, t) \in D_{m+1}\left(\mathcal{D}^{\eta}(\mathcal{R})\right) \subset D^{\xi}(\mathcal{R})$.

This completes the proof of (8) for all $m$. So (7) holds, and we are done.

Definition 9.6. A double tree $\mathcal{R}=\left(R^{+}, R^{\bullet}\right)$ on $A$ is said to be interpolable iff for some (countable) ordinal $\gamma$, one has $D^{\gamma}(\mathcal{R})=R^{+} \circ R^{\bullet}$.

Remark 9.7. If $\mathcal{R}$ is an interpolable double tree, then for every $y \in A^{\omega}$ there is at most one infinite branch in $\operatorname{Seq}(A)$ for $R(y)$.

Proof. If not, then we could find two sequences $\left(s_{k}\right)$ and $\left(t_{k}\right)$ generating two different branches and satisfying $s_{0} \preceq t_{0} \preceq s_{1} \preceq \cdots \preceq s_{k} \preceq t_{k} \preceq s_{k+1} \preceq \cdots \preceq y$. Since $s_{k} R s_{k+1}$ and $t_{k} R t_{k+1}$, it is easily checked by induction on $\xi$ that $\left(s_{k}, t_{k}\right)$ and $\left(t_{k}, s_{k+1}\right)$ are in $D^{\xi}(\mathcal{R})$ for all $k$. Then, since $R=D^{\gamma}(\mathcal{R})$, we get $s_{k} R t_{k}$ and $t_{k} R s_{k+1}$, and this means that $\left(s_{k}\right)$ and $\left(t_{k}\right)$ generate the same branch for $R(y)$, a contradiction.

We now prove that in some way the family $\left(D^{\xi}(\mathcal{R})\right)_{\xi}$ we just constructed is the least one with these properties.

Lemma 9.8. Let $\mathcal{R}$ be a double tree on $A$, and $\left(S^{\xi}\right)_{\xi \leq \gamma}$ a transfinite family of tree relations on $\operatorname{Seq}(A)$ satisfying :

i) $D^{0}(\mathcal{R}) \subset S^{0}$ and $S^{\gamma}=R$,

ii) for $\eta \leq \xi, R \subset S^{\xi} \subset S^{\eta}$,

iii) if $s \preceq t \preceq u, s S^{\xi+1} u$ and $t S^{\xi} u$, then $s S^{\xi+1} t$, and

iv) $S^{\lambda}=\bigcap_{\xi<\lambda} S^{\xi}$ for limit $\lambda$.

Then $D^{\xi}(\mathcal{R}) \subset S^{\xi}$ for all $\xi$, and $D^{\gamma}(\mathcal{R})=R$.

Proof. We show by induction on $\xi$ that $D^{\xi}(\mathcal{R}) \subset S^{\xi}$. For $\xi=0$ this is hypothesis i).

For limit $\lambda$, if $D^{\xi}(\mathcal{R}) \subset S^{\xi}$ for all $\xi<\lambda$, we clearly have

$$
D^{\lambda}(\mathcal{R})=\bigcap_{\xi<\lambda} D^{\xi}(\mathcal{R}) \subset \bigcap_{\xi<\lambda} S^{\xi}=S^{\lambda} .
$$

Finally, if $D^{\xi}(\mathcal{R}) \subset S^{\xi}$, we show by induction on $m$ that $D_{m}\left(D^{\xi}(\mathcal{R})\right) \subset S^{\xi+1}$. This is obvious for $m=0$. If it is true for $m$, assume $(s, t) \in D_{m+1}\left(D^{\xi}(\mathcal{R})\right)$ : there are by definition $u$ and $u^{\prime}$ such that $(s, u) \in D_{m}\left(D^{\xi}(\mathcal{R})\right), u \preceq t \preceq u^{\prime}, u R u^{\prime}$ and $\left.\left(t, u^{\prime}\right) \in D^{\xi}(\mathcal{R})\right)$. Then $s S^{\xi+1} u$ by the inductive hypothesis and $u S^{\xi+1} u^{\prime}$ by ii); hence $s S^{\xi+1} u^{\prime}$, since $S^{\xi+1}$ is a tree relation. So iii) applied to $s, t$, and $u^{\prime}$ yields $s S^{\xi+1} t$. Thus $D^{\xi+1}(\mathcal{R})=\bigcup_{m} D_{m}\left(D^{\xi}(\mathcal{R})\right) \subset S^{\xi+1}$, and this completes the proof that $D^{\xi}(\mathcal{R}) \subset S^{\xi}$.

Then for $\xi=\gamma$ we get $R \subset D^{\gamma}(\mathcal{R}) \subset S^{\gamma}=R$, and so $D^{\gamma}(\mathcal{R})=R$.

Example 9.9. In Examples 2.4 and 2.5 one has $D^{0}(\mathcal{R})=R$, and $\mathcal{R}$ is interpolable.

Theorem 9.10. If $\left(\mathcal{R}_{j}\right)$ is a sequence of interpolable double trees, then $\mathcal{R}=\bigotimes \mathcal{R}_{j}$ is interpolable.

By hypothesis, there are ordinals $\left(\gamma_{j}\right)$ such that $D^{\gamma_{j}}\left(\mathcal{R}_{j}\right)=R_{j}$ for all $j$. Define the increasing sequence $\left(\delta_{p}\right)$ of ordinals by $\delta_{0}=0$ and $\delta_{p+1}=\delta_{p}+\gamma_{p}+3$. We shall prove that $D^{\delta}(\mathcal{R})=R$ holds for $\delta=\sup _{p} \delta_{p}$. This will follow from the next lemma. 
Lemma 9.11. Assume $\rho(s)=\left(s_{0}, s_{1}, \ldots, s_{k}\right), \rho(t)=\left(t_{0}, t_{1}, \ldots, t_{\ell}\right)$ and $(s, t) \in$ $D^{\delta_{p}}(\mathcal{R})$. Then:

i) If $0 \leq k=w(s)<p$, then $s$ Rt.

ii) If $k=w(s) \geq p$, then $\ell=w(t) \geq p$ and $s_{i} R_{i}^{+} t_{i}$ for all $i<p$.

This lemma in turn will be proved by induction on $p$ using the following eight claims.

Claim 1. If $w(s) \geq p$ and $(s, t) \in D^{\delta_{p}}(\mathcal{R})$, then $w(t) \geq p$ and $\rho_{p}(s) \preceq \rho_{p}(t)$.

Claim 2. If $w(s) \geq p$ and $(s, t) \in D^{\delta_{p}+\xi}(\mathcal{R})$, then $\left(\rho_{p}(s), \rho_{p}(t)\right) \in D^{\xi}\left(\mathcal{R}_{p}\right)$.

Claim 3. If $u \preceq t, u R u^{\prime}, w(u) \geq p$ and $\left(t, u^{\prime}\right) \in D^{\delta_{p}+\gamma_{p}}(\mathcal{R})$, then $\rho_{p}(u) R_{p} \rho_{p}(t)$ and $\rho_{p}(t) R_{p} \rho_{p}\left(u^{\prime}\right)$.

Claim 4. If $w(s)>p, s_{p} R_{p}^{\bullet} t_{p}$ and $(s, t) \in D^{\delta_{p}+\gamma_{p}+1}(\mathcal{R})$, then $\rho_{p}(s)=\rho_{p}(t)$.

Claim 5. If $\rho(s)=\left(s_{0}, s_{1}, \ldots, s_{p}\right), \rho\left(s^{\prime}\right)=\left(s_{0}, s_{1}, \ldots, s_{\ell}\right)$ and $p<\ell$, then $\left(s, s^{\prime}\right) \notin$ $D^{\delta_{p}+\gamma_{p}+2}(\mathcal{R})$.

Claim 6. If $w(s)=p$ and $(s, t) \in D^{\delta_{p}+\gamma_{p}+2}(\mathcal{R})$, then $\rho_{p}(s) R_{p}^{\bullet} \rho_{p}(t)$.

Claim 7. If $w(s)>p=w(t)$, then $(s, t) \notin D^{\delta_{p}+\gamma_{p}+3}(\mathcal{R})$.

Claim 8. If $w(s)>p$ and $(s, t) \in D^{\delta_{p}+\gamma_{p}+3}(\mathcal{R})$, then $\rho_{p}(s) R_{p}^{+} \rho_{p}(t)$.

Proof of Theorem 9.10. Assume $(s, t) \in D^{\delta}(\mathcal{R})$ and let $p=w(s)+1$. Since $\delta \geq \delta_{p}$, we have $(s, t) \in D^{\delta_{p}}(\mathcal{R})$ and $w(s)<p$; hence $s R t$ by Lemma 9.11. It follows that $R \subset D^{\delta}(\mathcal{R}) \subset R$; hence $R=D^{\delta}(\mathcal{R})$, which shows that $\mathcal{R}$ is interpolable.

Proof of Lemma9.11. This is done by induction on $p$.

For $p=0$, the only thing we have to prove is that if $s \neq \emptyset$ and $(s, t) \in D^{0}(\mathcal{R})$, then $t \neq \emptyset$. But since $(s, t) \in D^{0}(\mathcal{R})$ means that $s \preceq t$, this is obvious.

Now we assume that Lemma 9.11 and Claims 5 - 8 hold for $p$, and show that Lemma 9.11 holds for $p+1$.

If $(s, t) \in D^{\delta_{p+1}}(\mathcal{R})=D^{\delta_{p}+\gamma_{p}+3}(\mathcal{R})$ and $w(s)<p+1$, then

- either $w(s)<p$, and, since $\delta_{p+1} \geq \delta_{p}$ and Lemma 9.11 i) holds for $p$, we get $s R t$,

- or $w(s)=p$. Then $w(t) \geq p$ and $s_{j} R_{j}^{+} t_{j}$ for $j<p$, since Lemma9.11 ii) holds for $p$. Moreover, $s_{p} R_{p}^{\bullet} t_{p}$ by Claim [6] and if $s \neq t$, then $s_{p} \neq t_{p}$ by Claim [5. Then it follows from Lemma 8.4 that $s R t$. This completes the proof of Lemma 9.11 i) for $p+1$.

Assume now that $(s, t) \in D^{\delta_{p+1}}(\mathcal{R})=D^{\delta_{p}+\gamma_{p}+3}(\mathcal{R})$ and $w(s) \geq p+1$. Then $w(t) \geq p$ by Lemma 9.11 ii) for $p$, and $w(t) \neq p$ by Claim 7 . We conclude that $w(t) \geq p+1$.

We have $s_{j} R_{j}^{+} t_{j}$ for $j<p$ by Lemma 9.11 ii) for $p$, and $s_{p} R_{j}^{+} t_{p}$ by Claim 8 It follows that $s_{j} R_{j}^{+} t_{j}$ for $j<p+1$. This completes the proof of Lemma 9.11 ii) for $p+1$.

We finish by proving Claims $1-8$ for $p$ from Lemma 9.11 for $p$.

Proof of Claim 1 Let $\rho(s)=\left(s_{0}, s_{1}, \ldots, s_{k}\right)$ and $\rho(t)=\left(t_{0}, t_{1}, \ldots, t_{\ell}\right)$. Since $k=$ $w(s) \geq p$, we know by Lemma 9.11 that $\ell=w(t) \geq p$.

If $p=0$, we have $s \neq \emptyset$, and the hypothesis $(s, t) \in D^{0}(\mathcal{R})$ gives $s \preceq t$ and $\left|s_{0}\right| \leq\left|t_{0}\right|$, which in turn give $s_{0} \preceq t_{0}$, since $s_{0} \preceq s \preceq t$ and $t_{0} \preceq t$. 
If $p>0$, Lemma 9.11 states that $s_{p-1} R_{p-1}^{+} t_{p-1}$. Then

$$
\left|s_{p}\right|=h_{p-1}^{+}\left(s_{p-1}\right)-1 \leq h_{p-1}^{+}\left(t_{p-1}\right)-1=\left|t_{p}\right|,
$$

and since again $s_{p} \preceq s \preceq t$ and $t_{p} \preceq t$, it follows that $s_{p} \preceq t_{p}$.

Proof of Claim 2. This is done by induction on $\xi$. Let $s_{p}=\rho_{p}(s)$ and $t_{p}=\rho_{p}(t)$.

For $\xi=0$, what we have to prove is the following: "if $k=w(s) \geq p$ and $(s, t) \in D^{\delta_{p}}(\mathcal{R})$, then $s_{p} \preceq t_{p}$ ". But this follows from Claim 1]

Assume now $\lambda$ is a limit ordinal and Claim 2 holds for all $\xi<\lambda$. If $(s, t) \in$ $D^{\delta_{p}+\lambda}(\mathcal{R})$, then $\left(s_{p}, t_{p}\right) \in \bigcap_{\xi<\lambda} D^{\xi}\left(\mathcal{R}_{p}\right)=D^{\lambda}\left(\mathcal{R}_{p}\right)$.

Finally, assume Claim 2 holds for $\xi$. We will prove by induction on $m$ that if $(s, t) \in D_{m}\left(D^{\delta_{p}+\xi}(\mathcal{R})\right)$, then $\left(s_{p}, t_{p}\right) \in D^{\xi+1}\left(\mathcal{R}_{p}\right)$. It is obvious for $m=0$, since we then have $s=t$, and hence $s_{p}=t_{p}$. If this is true for $m$ and $(s, t) \in$ $D_{m+1}\left(D^{\delta_{p}+\xi}(\mathcal{R})\right)$, then there are $u$ and $u^{\prime}$ such that $u \preceq t, u R u^{\prime},(s, u) \in$ $D_{m}\left(D^{\delta_{p}+\xi}(\mathcal{R})\right)$ and $\left(t, u^{\prime}\right) \in D^{\delta_{p}+\xi}(\mathcal{R})$.

By Lemma 9.11 we know that $w(u) \geq p$, and by the inductive hypothesis on $\xi$ that $\left(t_{p}, \rho_{p}\left(u^{\prime}\right)\right) \in D^{\xi}\left(\mathcal{R}_{p}\right)$. Since $u R u^{\prime}$, we have $w\left(u^{\prime}\right) \geq w(u)$, and it follows from Lemma 8.4 that $\rho_{p}(u) R_{p} \rho_{p}\left(u^{\prime}\right)$. Moreover, since $u \preceq t, u R u^{\prime}$ and $\left(t, u^{\prime}\right) \in$ $D^{\delta_{p}}(\mathcal{R})$, we know that $(u, t) \in D^{\delta_{p}+1}(\mathcal{R}) \subset D^{\delta_{p}}(\mathcal{R})$; hence, by Claim $1, \rho_{p}(u) \preceq t_{p}$.

Thus $\left(\rho_{p}(u), t_{p}\right) \in D^{\xi+1}\left(\mathcal{R}_{p}\right)$. Also, since $\left(s_{p}, \rho_{p}(u)\right) \in D^{\xi+1}\left(\mathcal{R}_{p}\right)$ by the inductive hypothesis on $m$, we get $\left(s_{p}, t_{p}\right) \in D^{\xi+1}\left(\mathcal{R}_{p}\right)$. This completes the proof of Claim [2.

Proof of Claim 3. Since $u R u^{\prime}$, we have $w\left(u^{\prime}\right) \geq w(u) \geq p$. Moreover, since $u \preceq t$ and $\left(t, u^{\prime}\right) \in D^{\delta_{p}+\gamma_{p}}(\mathcal{R})$, it follows from the definition that $(u, t) \in D^{\delta_{p}+\gamma_{p}+1}(\overline{\mathcal{R}})$, and from Lemma 9.11 that $w(t) \geq p$. Since $D^{\gamma_{p}}\left(\mathcal{R}_{p}\right)=R_{p}$, it follows immediately from Claim 2 for $\xi=\gamma_{p}$ that $\left(\rho_{p}(t), \rho_{p}\left(u^{\prime}\right)\right) \in D^{\gamma_{p}}\left(\mathcal{R}_{p}\right)=R_{p}$, and that $\left(\rho_{p}(u), \rho_{p}(t)\right) \in D^{\gamma_{p}}\left(\mathcal{R}_{p}\right)=R_{p}$.

So $\rho_{p}(u) R_{p} \rho_{p}(t)$ and $\rho_{p}(t) R_{p} \rho_{p}\left(u^{\prime}\right)$.

Proof of Claim 4. Let $\rho(s)=\left(s_{0}, s_{1}, \ldots, s_{k}\right)$ and $\rho(t)=\left(t_{0}, t_{1}, \ldots, t_{\ell}\right)$. We prove by induction on $m$ that if $w(s)>p,(s, t) \in D_{m}\left(D^{\delta_{p}+\gamma_{p}}(\mathcal{R})\right)$ and $s_{p} R_{p}^{\bullet} t_{p}$, then $s_{p}=t_{p}$. This is obvious for $m=0$, since then $s_{p}=t_{p}$.

If it is true for $m$, if $s_{p} R_{p}^{\bullet} t_{p}$ and if $(s, t) \in D_{m+1}\left(D^{\delta_{p}+\gamma_{p}}(\mathcal{R})\right)$ with $w(s)>p$, then there are $u$ and $u^{\prime}$ such that $(s, u) \in D_{m}\left(D^{\delta_{p}+\gamma_{p}}(\mathcal{R})\right), u \preceq t, u R u^{\prime}$ and $(t, u) \in D^{\delta_{p}+\gamma_{p}}(\mathcal{R})$.

If $s=u$, we have $w\left(u^{\prime}\right) \geq w(u)=w(s)=k>p$, and since $u R u^{\prime}$, we have $s_{p}=\rho_{p}(u) R_{p}^{+} \rho_{p}\left(u^{\prime}\right)$. By Claim 3, we know that $s_{p} R_{p} t_{p}$. Then it follows from Lemma 7.5 that $s_{p} R_{p}^{+} t_{p}$, and then from Lemma 2.6 a) that $s_{p}=t_{p}$.

If $s \neq u$ and $(s, u) \in D_{m}\left(D^{\delta_{p}+\gamma_{p}}(\mathcal{R})\right)$, we have $s_{p} R_{p} \rho_{p}(u)$. By Claim 3 we know that $t_{p} R_{p} \rho_{p}\left(u^{\prime}\right)$ and $\rho_{p}(u) R_{p} t_{p}$. Since $s_{p} R_{p}^{\bullet} t_{p}$, it follows from Lemma 7.6 that $s_{p} R_{p}^{\bullet} \rho_{p}(u)$, and the inductive hypothesis then gives $s_{p}=\rho_{p}(u)$. It follows in turn that $w(s) \leq w(u)$; indeed, we have $s_{j}=\rho_{j}(u)$ for $j \leq p$ and if we had $w(s)>w(u)$ we would have

$$
|s|=\left|s_{0}\right| \frac{\left|s_{0}\right|+1}{2}+w(s)+1>\left|s_{0}\right| \frac{\left|s_{0}\right|+1}{2}+w(u)+1=|u|
$$

in contradiction with $s \preceq u$. Thus we have $\rho_{p}(u) R_{p}^{+} \rho_{p}\left(u^{\prime}\right)$. We conclude from Lemma 7.5 that $s_{p}=\rho_{p}(u) R_{p}^{+} t_{p}$. It follows from Lemma 2.6]a) that $s_{p}=t_{p}$. This completes the proof of Claim 4 . 
Proof of Claim 5. Assume that $\left(s, s^{\prime}\right) \in D^{\delta_{p}+\gamma_{p}+2}(\mathcal{R})$ with $\rho(s)=\left(s_{0}, s_{1}, \ldots, s_{p}\right)$, $\rho\left(s^{\prime}\right)=\left(s_{0}, s_{1}, \ldots, s_{\ell}\right)$ and $\ell>p$. Without loss of generality we assume that $\ell$ is minimal, that is, $(s, u) \notin D^{\delta_{p}+\gamma_{p}+2}(\mathcal{R})$ if $\rho(u)=\left(s_{0}, s_{1}, \ldots, s_{\ell^{\prime}}\right)$ for $p<\ell^{\prime}<\ell$.

Since $\left(s, s^{\prime}\right) \in D^{\delta_{p}+\gamma_{p}+2}(\mathcal{R})$, there are $u$ and $u^{\prime}$ such that $u R u^{\prime}, u \preceq s^{\prime}, u \neq s^{\prime}$, $(s, u) \in D^{\delta_{p}+\gamma_{p}+2}(\mathcal{R})$ and $\left(s^{\prime}, u^{\prime}\right) \in D^{\delta_{p}+\gamma_{p}+1}(\mathcal{R})$. Since $s \preceq u \preceq s^{\prime}$, we have necessarily $\left|s_{0}\right| \leq\left|\rho_{0}(u)\right| \leq\left|\rho_{0}\left(s^{\prime}\right)\right|=\left|s_{0}\right|$; hence $\left|\rho_{0}(u)\right|=\left|s_{0}\right|$, and $s_{0}=\rho_{0}(u)$ since $s_{0} \preceq s \preceq s^{\prime}$ and $\rho_{0}(u) \preceq u \preceq s^{\prime}$. We conclude that $\rho(u)=\left(s_{0}, s_{1}, \ldots, s_{\ell^{\prime}}\right)$ for some $\ell^{\prime}$ such that $p \leq \ell^{\prime}<\ell$. But since $(s, u) \in D^{\delta_{p}+\gamma_{p}+2}(\mathcal{R})$, the minimality of $\ell$ implies $\ell^{\prime}=p$.

Then $u=s$ and $s_{p} R_{p}^{\bullet} \rho_{p}\left(u^{\prime}\right)$. Moreover, since $w\left(s^{\prime}\right)=\ell>p$ and $\left(s^{\prime}, u^{\prime}\right) \in$ $D^{\delta_{p}+\gamma_{p}+1}(\mathcal{R})$, it follows from Claim 4 that $s_{p}=\rho_{p}\left(u^{\prime}\right)$. We conclude that $\rho_{j}\left(u^{\prime}\right)=$ $s_{j}=\rho_{j}(u)$ for $j \leq p$. Since $u R u^{\prime}$, this is possible only if $u^{\prime}=u=s$. But then we get a contradiction between $s^{\prime} \preceq u^{\prime}$ and

$$
\left|s^{\prime}\right|=\left|s_{0}\right| \frac{\left|s_{0}\right|+1}{2}+\ell+1>\left|s_{0}\right| \frac{\left|s_{0}\right|+1}{2}+p+1=|u|=\left|u^{\prime}\right| .
$$

This completes the proof of Claim 5 .

Proof of Claim [6. Let $s_{p}=\rho_{p}(s)$ and $t_{p}=\rho_{p}(t)$. We prove by induction on $m$ that if $w(s)=p$ and $(s, t) \in D_{m}\left(D^{\delta_{p}+\gamma_{p}+1}(\mathcal{R})\right)$, then $s_{p} R_{p}^{\bullet} t_{p}$. It is obvious for $m=0$, since then $s_{p}=t_{p}$.

If it is true for $m$ and if $(s, t) \in D_{m+1}\left(D^{\delta_{p}+\gamma_{p}+1}(\mathcal{R})\right)$ with $w(s)=p$, there are $u$ and $u^{\prime}$ such that $(s, u) \in D_{m}\left(D^{\delta_{p}+\gamma_{p}+1}(\mathcal{R})\right), u \preceq t, u R u^{\prime}$ and $(t, u) \in$ $D^{\delta_{p}+\gamma_{p}+1}(\mathcal{R})$. Notice that, by Lemma 9.11, $w(u) \geq p$ and $w(t) \geq p$. By the inductive hypothesis we know that $s_{p} R_{p}^{\bullet} \rho_{p}(u)$. By Claim [3, we know that $t_{p} R_{p}$ $\rho_{p}\left(u^{\prime}\right)$, and that $\rho_{p}(u) R_{p} t_{p}$. Then:

- either $s_{p}=\rho_{p}(u)$. In this case we should have $\rho_{j}(u)=s_{j}$ for $j \leq p$; hence $s=u$ by Claim 5. Then, since $w(u)=w(s)=p$ and $u R u^{\prime}$, we have $s_{p}=\rho_{p}(u) R_{p}^{\bullet} \rho_{p}\left(u^{\prime}\right)$. Moreover, we have $\rho_{p}(u) R_{p} t_{p}$ and $t_{p} R_{p} \rho_{p}\left(u^{\prime}\right)$. It follows then from Lemma 7.6 that $s_{p}=\rho_{p}(u) R_{p}^{\bullet} t_{p}$.

- or $s_{p} \neq \rho_{p}(u)$. In this case we have $s_{p} R_{p}^{\bullet} \rho_{p}(u)$ and $\rho_{p}(u) R_{p} t_{p}$; hence $s_{p} R_{p}^{\bullet} t_{p}$ by Lemma 7.2

In both cases we conclude that $s_{p} R_{p}^{\bullet} t_{p}$, and the proof is complete.

Proof of Claim 7. Let $\rho(t)=\left(t_{0}, t_{1}, \ldots, t_{\ell}\right)$. We prove by induction on $m$ that if $(s, t) \in D_{m}\left(D^{\delta_{p}+\gamma_{p}+2}(\mathcal{R})\right)$ and $w(s)>p$, then $\ell=w(t)>p$. This is obvious when $m=0$, since then $s=t$.

Assume it is true for $m,(s, t) \in D_{m+1}\left(D^{\delta_{p}+\gamma_{p}+2}(\mathcal{R})\right)$ and $w(s)>p=w(t)$. Then there are $u$ and $u^{\prime}$ such that $u \preceq t, u R u^{\prime},(s, u) \in D_{m}\left(D^{\delta_{p}+\gamma_{p}+2}(\mathcal{R})\right)$ and $\left(t, u^{\prime}\right) \in D^{\delta_{p}+\gamma_{p}+2}(\mathcal{R})$. By the inductive hypothesis we have $w(u)>p$. Since $u R u^{\prime}$, it follows that $\rho_{p}(u) R_{p}^{+} \rho_{p}\left(u^{\prime}\right)$. By Claim 3 we have $\rho_{p}(u) R_{p} t_{p}$ and $t_{p} R_{p} \rho_{p}\left(u^{\prime}\right)$. Thus by Lemma 7.5 we conclude that $t_{p} R_{p}^{+} \rho_{p}\left(u^{\prime}\right)$.

Since $\left(t, u^{\prime}\right) \in D^{\delta_{p}+\gamma_{p}+2}(\mathcal{R})$, we have $t_{p} R_{p}^{\bullet} \rho_{p}\left(u^{\prime}\right)$ by Claim [6] It follows then from Lemma 2.6 a) that $t_{p}=\rho_{p}\left(u^{\prime}\right)$; hence $t_{j}=\rho_{j}\left(u^{\prime}\right)$ for $j \leq p$. Since $w\left(u^{\prime}\right) \geq$ $w(u)>p=w(t)$, this contradicts Claim 5 . This contradiction completes the proof of Claim 7 .

Proof of Claim 8. Let $s_{p}=\rho_{p}(s)$ and $t_{p}=\rho_{p}(t)$. We show by induction on $m$ that if $(s, t) \in D_{m}\left(D^{\delta_{p}+\gamma_{p}+2}(\mathcal{R})\right)$ and $w(s)>p$, then $s_{p} R_{p}^{+} t_{p}$. This is clear for $m=0$, since then $s_{p}=t_{p}$. 
Assume the statement is true for $m$, that $(s, t) \in D_{m+1}\left(D^{\delta_{p}+\gamma_{p}+2}(\mathcal{R})\right)$ and that $w(s)>p$. Then there are $u$ and $u^{\prime}$ such that $u \preceq t, u R u^{\prime},(s, u) \in$ $D_{m}\left(D^{\delta_{p}+\gamma_{p}+2}(\mathcal{R})\right)$ and $\left(t, u^{\prime}\right) \in D^{\delta_{p}+\gamma_{p}+2}(\mathcal{R})$.

By the inductive hypothesis we know that $s_{p} R_{p}^{+} \rho_{p}(u)$. Also, by Claim 7 we know that $w(u)>p$. Thus we deduce from $u R u^{\prime}$ that $\rho_{p}(u) R_{p}^{+} \rho_{p}\left(u^{\prime}\right)$. From Claim [3 we know that $\rho_{p}(u) R_{p} t_{p}$ and $t_{p} R_{p} \rho_{p}\left(u^{\prime}\right)$. It follows then from Lemma 7.5 that $\rho_{p}(u) R_{p}^{+} t_{p}$, hence that $s_{p} R_{p}^{+} t_{p}$, since $s_{p} R_{p}^{+} \rho_{p}(u)$. This completes the proof of Claim 8 .

Proof of Theorem 2.8. We prove by induction on $\xi$ that for every $\boldsymbol{\Pi}_{\xi}^{0}$ set $Y$ in $\mathbf{2}^{\omega}$, there exists a uniquely branching and interpolable double tree with kernel $Y$. This will ensure conditions a) and $b$ ) of Theorem 2.8.

For $\xi=1$, Example 2.4yields a double tree which is clearly uniquely branching and is interpolable by 9.9 .

If this is done for $\eta<\xi$, and if $Y$ is $\mathbf{\Pi}_{\xi}^{0}$, there is a sequence $\left(Y_{j}\right)$ of subsets of $\mathbf{2}^{\omega}$, each of them being $\boldsymbol{\Pi}_{\eta_{j}}^{0}$ for some $\eta_{j}<\xi$, such that $Y=\bigcap_{j}\left(\mathbf{2}^{\omega} \backslash Y_{j}\right)$. By the inductive hypothesis there are uniquely branching and interpolable double trees $\mathcal{R}_{j}$ with $\operatorname{ker}\left(\mathcal{R}_{j}\right)=Y_{j}$. Then if we define $\mathcal{R}=\bigotimes \mathcal{R}_{j}$, we know that $\mathcal{R}$ is uniquely branching by Theorem 8.6 , and that $Y=\operatorname{ker}(\mathcal{R})$ by Theorem 8.7. Finally, Theorem 9.10 shows that $\mathcal{R}$ is interpolable.

A careful inspection of the proof shows that if $Y$ is $\Pi_{\xi}^{0}(\alpha)$, the double tree we built is in $L(\alpha)$. Thus the proof is complete.

\section{REFERENCES}

1. J.P.R. Christensen, Necessary and sufficient conditions for the measurability of certain sets of closed sets, Math. Annaluen 200 (1973), 189-193 MR 48:12488

2. G. Debs and J. Saint Raymond, Compact covering mappings and game determinacy, Topology Appl. 68 (1996), 153-185 MR 96m:54067

3. G. Debs and J. Saint Raymond, Cofinal $\boldsymbol{\Sigma}_{1}^{1}$ and $\Pi_{1}^{1}$ subsets of $\omega^{\omega}$, Fund. Math. 159 (1999), 161-193 MR 2001g:03087

4. G. Debs and J. Saint Raymond, Compact covering mappings and cofinal families of compact subsets of a Borel set, Fund. Math. 167 (2001), 213-249 MR 2001k:03098

5. G. Debs and J. Saint Raymond, Applications semi-propres sur un espace borélien, C. R. Acad. Sci. Paris t. 232, Série I, (2001), 423-426 MR 2002a:28002

6. W. Just and H. Wicke, Some conditions under which tri-quotient or compact-covering maps are inductively perfect, Topology Appl. 55 (1994), 289-305 MR 95a:54051

7. Y.N. Moschovakis, Desriptive Set Theory (North Holland, Amsterdam, 1980) MR 82e:03002

8. A.V. Ostrovsky, On new classes of mappings associated with compact-covering mappings, Vestnik Moskov. Univ. Ser. I Mat. Mekh.1994, no. 4, 24-28; English transl., Moscow Univ. Math. Bull. 49 (1994), no. 4, 20-23. MR 96a:54013

9. J. Saint Raymond, Caractérisation d'espaces polonais, Séminaire d'Initiation à l'Analyse 1112èmes années, no 5 (1971-73) MR[57:12811]

Analyse Fonctionnelle, Institut de Mathématique de Jussieu, Boîte 186, 4, Place Jussieu, 75252 Paris Cedex 05, France

E-mail address: gad@ccr.jussieu.fr

Analyse Fonctionnelle, Institut de Mathématique de Jussieu, Boîte 186, 4, Place Jussieu, 75252 Paris Cedex 05, France

E-mail address: jsr@ccr.jussieu.fr 